DOE/ID/12277--Tl-Vol.1

DE83 000805

\title{
FEASIBILITY STUDY OF A CORN-TO-ETHANOL PLANT IN SARDIS, MISSISSIPPI
}

2

Volume I

JUNE 1982

Prepared by

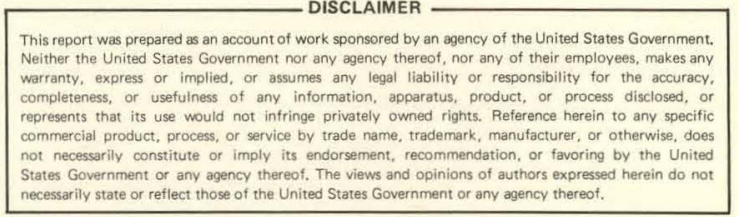

\section{Sun Belt Energy Corporation}

212 N. Main Street

Sardis, Mississippi 38666

Assisted by

Resource Planning Associates, Inc.

50 Church Street

Cambridge, Massachusetts 02138

and

Bechtel Petroleum, Inc.

1930 Bishop Lane

Louisville, Kentucky 40232

For the U.S. Department of Energy

Office of Alcohol Fuels

Under Grant No. DE-FG07-81ID12277 


\section{DISCLAIMER}

This report was prepared as an account of work sponsored by an agency of the United States Government. Neither the United States Government nor any agency Thereof, nor any of their employees, makes any warranty, express or implied, or assumes any legal liability or responsibility for the accuracy, completeness, or usefulness of any information, apparatus, product, or process disclosed, or represents that its use would not infringe privately owned rights. Reference herein to any specific commercial product, process, or service by trade name, trademark, manufacturer, or otherwise does not necessarily constitute or imply its endorsement, recommendation, or favoring by the United States Government or any agency thereof. The views and opinions of authors expressed herein do not necessarily state or reflect those of the United States Government or any agency thereof. 


\section{DISCLAIMER}

Portions of this document may be illegible in electronic image products. Images are produced from the best available original document. 


\title{
DISCLAIMER
}

This book was prepared as an account of work sponsored by an agency of the United States Government. Neither the United States Government nor any agency thereof, nor any of their employees, makes any warranty, express or implied, or assumes any legal liability or responsibility for the accuracy, completeness, or usefulness of any information, apparatus, product or process disclosed, or represents that its use would not infringe privately owned rights. References herein to any specific commercial product, process, or service by trade name, trademark, manufacturer, or otherwise, does not necessarilv constitute or imply its endorsement, recommendation, or favoring by the United States Government or any agency thereof. The views and opinions of authors expressed herein do not necessarily state or reflect those of the United States Government or any agency thereof.

Printed in the United States of America

\author{
Available from \\ National Technical Information Service \\ U.S. Department of Commerce \\ 5285 Port Royal Road \\ Springfield, VA 22161

\section{NTIS Price Codes Printed Copy A11} \\ Microfiche A01
}


Sun Belt Energy Corporation carried out a DOE-sponsored feasibility study for a corn-to-ethanol plant in Panola County, Mississippi. This area is well suited for the production of ethanol from corn, as it has a mild climate, a plentiful supply of wood fuel, and a well-developed agricultural infrastructure. The project was designed for 5 million gallons per year, using the ACR Process, a process proven in 6 plants now operating. It was determined to be technically feasible for this size. However, without a state financial incentive such as a gasoline excise tax or sales tax exemption, the plant is not economically feasible in Mississippi. Even though a $4 \notin$ per gallon federal excise tax exemption will likely remain, the economics without any other incentive are not strong enough to obtain financing or equity funds. While the Mississippi legislature decided not to consider a financial incentive in their 1982 session, an attempt will be made to introduce a proposal for a suitable exemption during the 1983 legislative session. Until then, the project is on hold. 


\section{Contents}

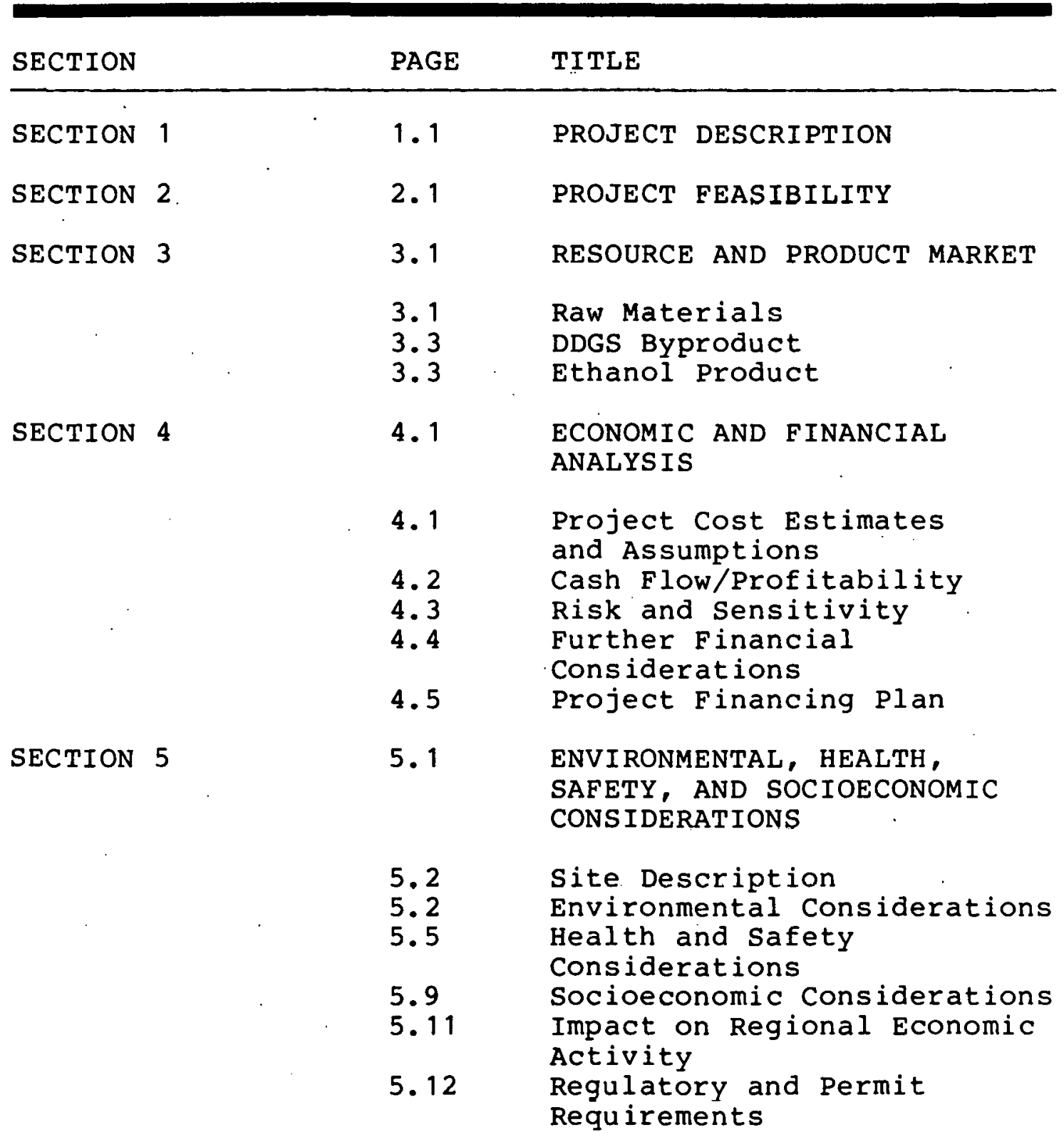


CONTENTS

SECTION 6

6.1

ORGANIZATION AND MANAGEMENT PLAN

6. 1 Schedule for Project

6.4 Implementation

6.5 Key Management Activities

APPENDIX A

PERMITS REQUIRING MANAGEMENT ATTENTION

APPENDIX B

COMPUTER MODEL SENSITIVITY RESULTS 


\section{Exhibits}

\begin{tabular}{|c|c|c|c|}
\hline \multicolumn{2}{|l|}{ SECTION } & \multirow{2}{*}{ No. } & \multirow{2}{*}{$\begin{array}{l}\text { TITLE } \\
\begin{array}{l}\text { Sun Belt Ethanol Plant Flow } \\
\text { Diagram }\end{array}\end{array}$} \\
\hline SECTION & 1 & & \\
\hline & & 1.2 & $\begin{array}{l}\text { Sun Belt Ethanol Plant } \\
\text { Proposed Plant Site } \\
\text { and Vicinity Map }\end{array}$ \\
\hline \multirow[t]{5}{*}{ SECTION } & 3 & 3.1 & $\begin{array}{l}\text { Letters of Interest in } \\
\text { Purchasing DDGS Output }\end{array}$ \\
\hline & & 3.2 & $\begin{array}{l}\text { Letter Concerning Potential } \\
\text { for Local Feedstocks }\end{array}$ \\
\hline & & 3.3 & Draft contract \\
\hline & & 3.4 & Draft Contract Language \\
\hline & & 3.5 & $\begin{array}{l}\text { Wood Services, Inc., wood } \\
\text { Supply study }\end{array}$ \\
\hline \multirow[t]{6}{*}{ SECTION } & 4 & 4.1 & $\begin{array}{l}\text { Sun Belt Ethanol Plant } \\
\text { Capital Cost Summary and } \\
\text { Annual operating Cost Summary }\end{array}$ \\
\hline & & 4.2 & $\begin{array}{l}\text { Financial Analysis of } \\
\text { Sun Belt Energy Corporation } \\
\text { Project }\end{array}$ \\
\hline & & 4.3 & Income and Cash Flow \\
\hline & & 4.4 & Balance sheet \\
\hline & & 4.5 & Sources and Uses of Funds \\
\hline & & 4.6 & $\begin{array}{l}\text { Sensitivity of Corn-to-Ethanol } \\
\text { Project Performance to Changes } \\
\text { in Corn and Ethanol Price }\end{array}$ \\
\hline
\end{tabular}


4.7

Downside Risk Analyses

5-Million GPY Corn-to-Ethanol Project

4.8a 10-Year Average Cash Prices

for Selected Grains and

Unleaded Gasoline

4.8b Long-Term Historical Cash

Price Trends for Selected

Grains

SECTION 5

5.1

Population and Economic Characteristics of Panola

County and Sardis, Mississippi

5.2 Economic Impact of Ethanol Plant

5.3 Meredith Letter on Economic Impacts

5.4 Annualized Increase in Economic Activity

5.5 Regulatory Requirements

5.6 Schedule for Regulatory Requirements

SECTION 6

6.1

Schedule of Major Project Implementation Activities

6. 2 Management Organization for Construction

6.3 Responsibilities for Key Construction Activities

6.4

Management Organization

for Plant Operation

6.5 Sun Belt Energy Corporation Plant Personnel Required 
Sun Belt Energy Corporation (Sun Belt) is dedicated to the planning, construction, and operation of a fuel alcohol plant in Panola County, Mississippi. The three principals of the corporation, Mr. Billie Shepard, Mr. Harold Graham, and Mr. Fred Klyce, are all residents of Panola county and are fully committed to the successful development of the project. That committment is evidenced by a substantial investment of time and money by the principals in project planning and pre-feasibility study efforts to date.

Mr. Shepard, President of Sun Belt, is a retail gasoline dealer for Chevron U.S.A. and has major farming and livestock interests in the county. He is a former president of the Panola County Farm Bureau and has served as personal advisor to the Governor of Mississippi.

Mr. Graham, Vice President of Sun Belt, has substantial experience in agriculture and agricultural administration. He owns and operates a large soybean farm and cattle ranch, and for several years was a member of the Panola County Farmers Home Administration. In addition, Mr. Graham has experience in energy facility construction.

Mr. Klyce, Secretary-Treasurer of Sun Belt, has served as President of the Panola County Bank since 1964, is President of the Mississippi Cattlemen's Association, and is a former chairman of the Agricultural Committee of the Mississippi Bankers Association. As a local farmer and rancher, Mr. Klyce is thoroughly acquainted with local business and economic conditions.

To achieve its goal, Sun Belt has retained substantial professional services. Bechtel Petroleum, Incorporated, has conducted an engineering feasibility study that has verified the feasibility of the ACR process on sun Belt!s site. Within the past two years, Bechtel Petroleum, a leader in the engineering design of fuel alcohol plants, 
has conducted more than a dozen fuel alcohol plant feasibility studies throughout the United States. Moreover, the firm has completed final designs of two projects specifically using the ACR process: a 2.5-million gallon per year (mgy) facility in Oklahoma, which began operations in 1981; and a 20-MGY facility in Kentucky, which is under construction. Carothers and Carothers, a Mississippi general contractor with extensive experience in the design and construction of industrial facilities, has assessed the transportation and geotechnical requirements for the proposed plant.

Resource Planning Associates, Inc. (RPA), a management consulting firm specializing in energy and natural resources, has assisted sun Belt in the market, resource supply, financial, and impact analyses for the project.

The major process steps of the fuel alcohol plant are shown in schematic form in Exhibit 1.1. Briefly, grain is delivered to the storage silos at a rate of 5,700 bushels per day and is then milled to produce a fine meal. The meal goes through a number of processes, including cooking, fermentation, and distillation, resulting in the production of ethanol and a byproduct, distillers' dried grain solubles (DDGS). The DDGS has a high protein content and can be sold as cattle feed.

The plant's equilibrium power capacity requirements are about 22,000 pounds per hour of steam and $1 \mathrm{MW}$ of electric power.

Panola County, Mississippi, is well suited for the production of ethanol from corn and other grain. It enjoys a fine agricultural climate, with an average of 300 days of sunshine and less than 20 days of freesing weather each year. An adequate supply of grain and wood fuel, the presence of a well-developed agricultural infrastructure, and easily accessible road, rail, and water transportation systems provide sun Belt with an excellent operating environment.

Sun Belt proposes to build the grain-to-ethanol plant on a 120-acre industrial park site. The site is between U.S. Highway 51 and Interstate 55, about one mile north of Sardis, Mississippi, and $45 \mathrm{miles}$ south of Memphis, Tennessee (see Exhibit 1.2). It is adjacent to mainline trackage of the Illinois Central Gulf Railroad, and is 
2 miles from Interstate Highway 55 and 30 miles from the Mississippi River. Sun Belt has acquired an option to purchase the property.

The site is located on gently sloping (2-5 percent grade) Grenada soil.* According to the Soil Conservation Service, U.S.D.A., "This area is not in a flood plain and would be suitable for the kind of light industry proposed.... No water problem exists. The area is moderately well drained."

Other important site characteristics include the following:

- It is zoned for industrial use

- There is an electric power substation within 1.5 miles

- Water supply and sewage disposal systems are available to the site.

Wood is planned as the fuel to fire the boilers that provide steam for the alcohol production process. The fuel requirements of such a facility would be 112 tons of wood per day. There are 500-1,000 tons per day of wood waste (low-quality timber, wood chips, and sawdust) currently available from nearby sawmills (e.g.. Floyd Lumber Company, Yunt Lumber Company, and Tallahatchie Hardware, Inc.), plus 1,000-2,000 tons per day of locally harvestable timber.

* A site survey was made, topographic maps were obtained, and relevant soils factors were evaluated. The final plant layout (see Volume II) reflects this evaluation. 
Exhibit 1.1

Sun Belt Ethanol Plant Flow Diagram

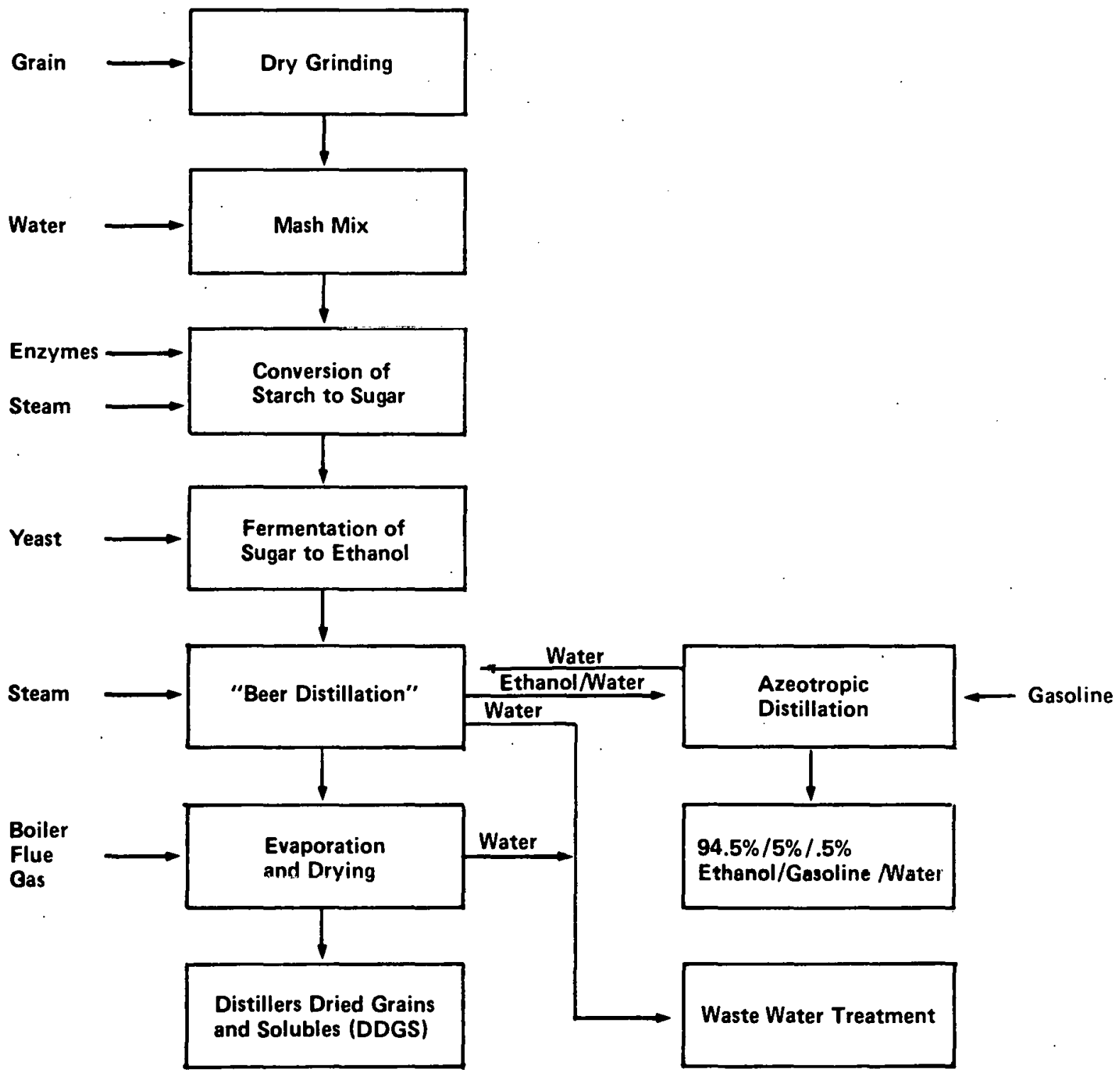


Exhibit 1.2

Sun Belt Ethanol Plant

Proposed Plant Site

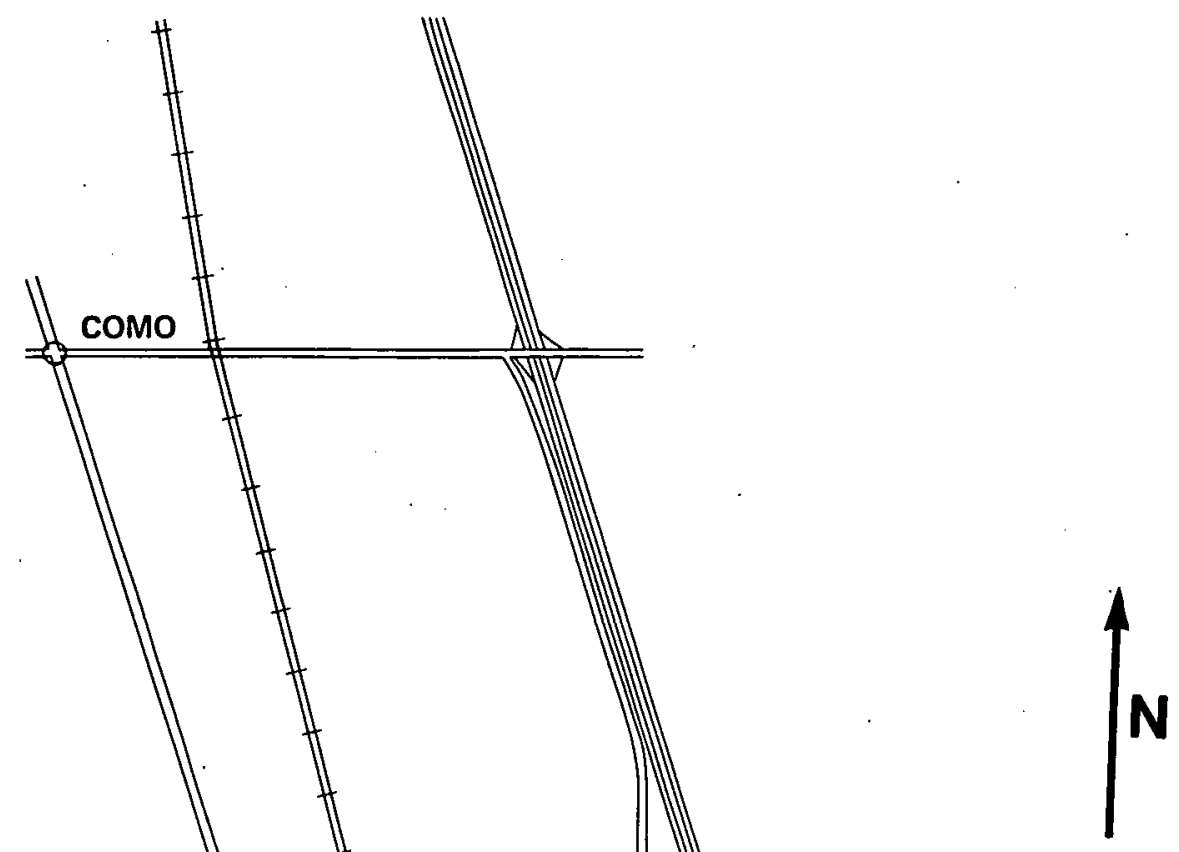

\section{Scale}
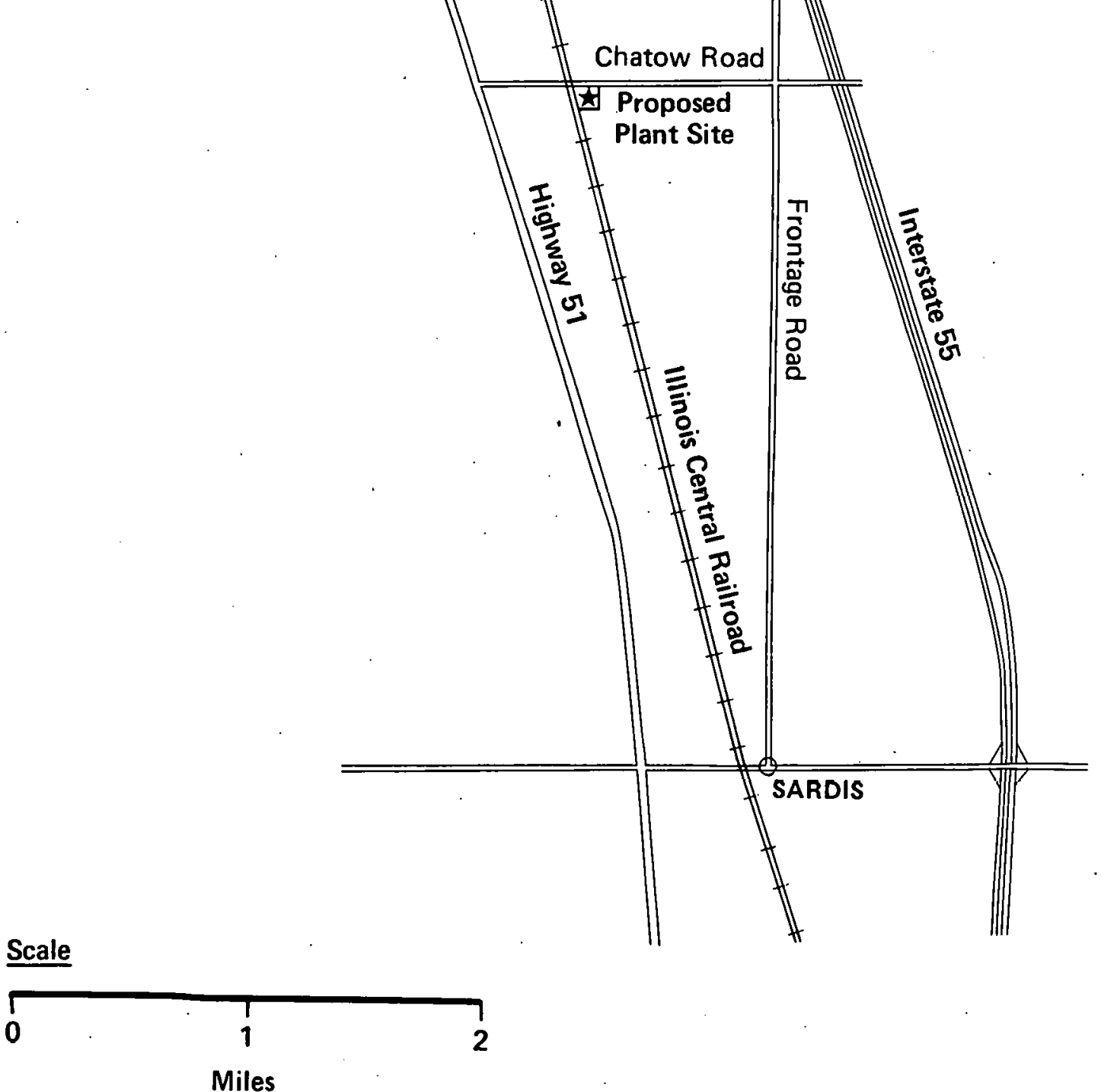
Exhibit 1.2 (continued)

Sun Belt Ethanol Plant

Vicinity Map

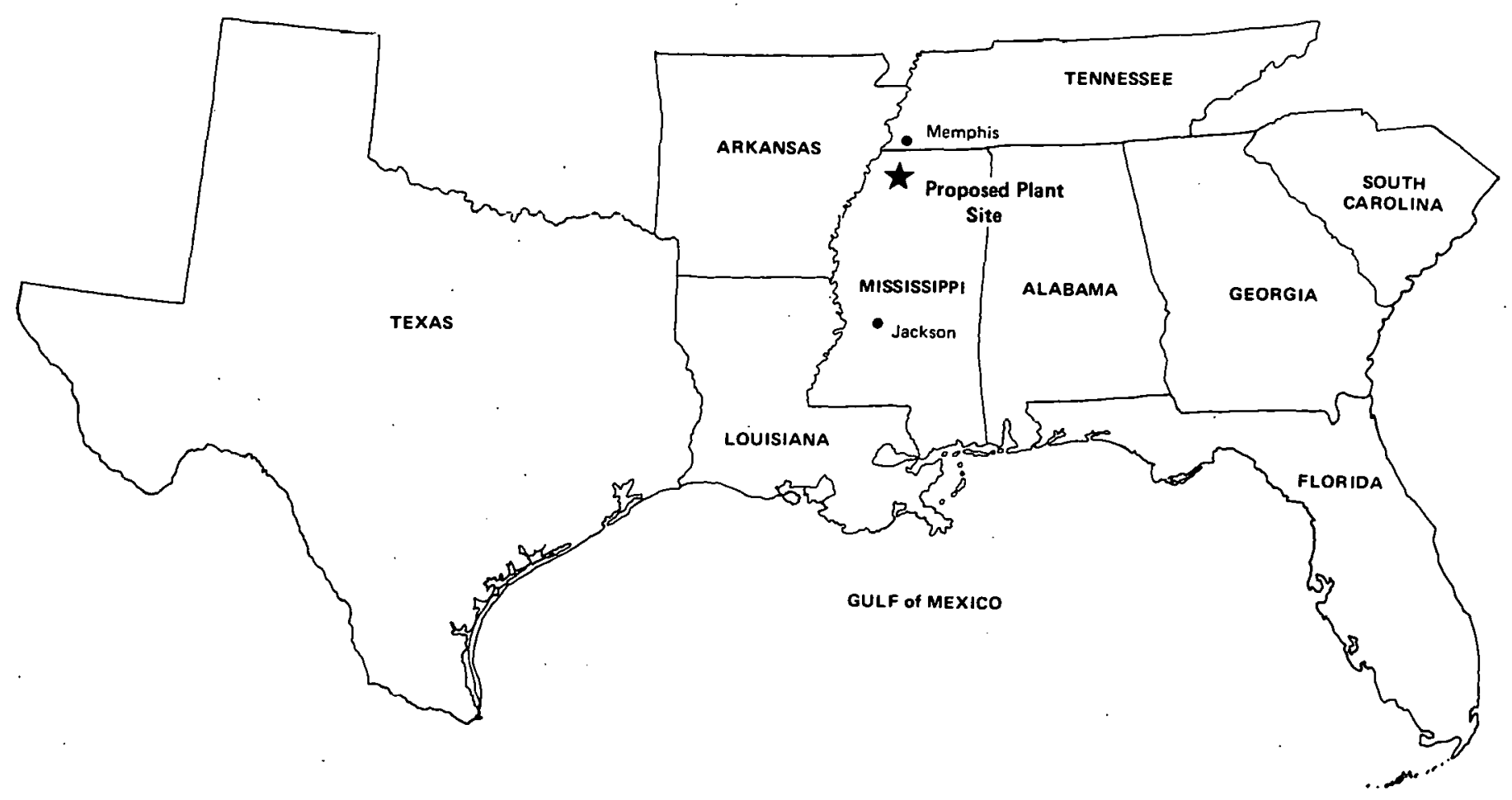


The project, sited in sardis, has been determined technically feasible, as well as feasible from a regulatory point of view (environmental and associated impacts). However, without a state financial incentive, such as a state gasoline excise tax or sales tax exemption, the project is not economically feasible at the sardis site, or any other site in Mississippi. As of now, the state legislature has decided not to consider such a financial incentive. The factors affecting the feasibility of the project are summarized below, and discussed in detail in the report sections that follow.

\section{Technical Feasibility}

The plant, as designed for the sardis site, has been determined by Bechtel petroleum to be technically feasible. The capital and operating costs are based on a previously proven design of a 2.5-milition gallon per year plant, scaled up for the 5-milition gallon per year capacity of the proposed plant. The local transportation systems and utilities can physically accommodate the supply of resources, satisfactory operation of the plant, and movement of the product. The technical feasibility is discussed in detail in Volume II of this report.

Economic/Financial

Feasibility

Without a state financial incentive, the economic feasibility of the proposed plant in Mississippi is not strong enough to obtain financing from any type of financing source. Likewise, the economic feasibility is not strong enough to attract the necessary equity funds. While the Mississippi legislature decided not to consider a financial incentive in the 1982 session just completed, an attempt will be made to introduce a proposal for a suitable exemption in the 1983 session. The project cannot 
move forward before then unless the plan is shifted to another state with a sufficient incentive. Beyond the scope of this feasibility study, Sun Belt is exploring such opportunities. The economic/financial feasibility is discussed in sections 3 and 4 : 


\subsection{RAW MATERIALS}

\section{Grain Feedstocks}

The plant is designed for corn. Grain sorghum (milo) or wheat can be used, with some equipment changes, e.g., the screen size for the hammermill. Fifteen days of on-site grain storage will be provided, supplemented by another 30 days' storage in local cooperative and farm facilities. The on-farm storage facilities and local elevators assure continuous delivery for the plant.

The initial supply ( 2 million bushels per year) will be supplied by a large grain company at a price tied by formula to an established grain exchange price. I.S. Joseph has expressed an interest in supplying the corn and purchasing the DDGS output from the plant; International Multifoods and Ralston Purina have also expressed interest in purchasing the plant's DDGS output, priced as delivered to the plant site.*

Transportation systems for delivering the grain are excellent. Sardis is located $45 \mathrm{miles}$ south of Memphis. There are several trucking companies, including Beard Truck Lines and Rebel Motor Freight. Further, large grain companies have multi-state trucking firms under contract for continuous delivery of their products. As shown in Exhibit 1.2, the site is well situated with regard to major highway and railroad systems.

Over time, as local crops can be shifted, local grain supplies will be phased in through a combination of farm cooperative and individual farm supply channels. Based on

* Exhibit 3.1 contains related letters of interest. 
an analysis by the Mississippi Agricultural Extension Service, the local area clearly has the grain supply capability. See Exhibit 3.2. Exhibit 3.3 contains a draft contract to be used. Further, where the economics would be enhanced by it, distressed grain and low-price grain opportunities will be exploited to minimize the cost of the feedstock. Sun Belt has made contact with two sources of such lower-cost grain, one in Memphis and one in Houston, both of which have continuous supplies of such special grain.

Finally, in order to properly manage the commodity nature of the grain price, sun Belt will include grain-brokering expertise in its plant organization. It is sun Belt's intent to buy options to hedge against adverse changes in grain prices that could increase the cost and decrease the profits of the plant operation.

Fuel

Wood is planned as the boiler fuel, with 112 tons per day to be supplied from nearby sawmills and harvestable supplies. Due to the large quantity of locally available wood, only three days' on-site storage will be provided.

An extensive wood-supply. survey has been conducted, which indicates the immediate availability of 500-1,000 tons per day of waste wood from nearby sawmills, and 1,000-2,000 tons per day of locally harvestable timber. Competition for these wood supplies is expected to be limited, as there are no sales now of such wood supplies, and the 112 tons per day required by the plant is small compared to the total available supply of wood. That is, a number of inductrial plants using wood as a fucl could be con. structed in northern Mississippi without demand even approaching the available supply of wood.

A contract has been preliminarily negotiated at $\$ 10.70$ per ton (1982 dollars), delivered and chipped, subject to an inflation escalation. The contract has been negotiated with Wood Services, Inc.*

* Exhibit 3.4 contains the draft contract language, and Exhibit 3.5 contains the wood supply study results. 


\subsection{DDGS BYPRODUCT}

The DDGS byproduct (16-17 thousand tons per year) will initially be sold to a large grain company, priced according to an established DDGS exchange price. Over time, as local markets develop, the DDGS will be sold to local cattlemen.

At some point, wet stillage will be considered for direct sale on a daily basis. The Mississippi Agricultural Cooperative Extension Service will assist Sun Belt in the research required to determine the best mixtures of stillage for each class of livestock feed. Mr. Joe Hensley, Panola County Agent, and Dr. Robert stewart, animal nutritionist at Mississippi State University, have agreed to assist Sun Belt.

Since wet stillage has a short shelf life and there are no good means of disposing spoiled product, there is no direct daily market for it in Panola County.* Once the plant starts up, small amounts of wet stillage will be analyzed and local purchase interest explored. If there is interest, the transportation would be provided by the purchaser. The most likely purchaser would be a feedlot. However, currently there are none in Panola county because of poor feedlot economics at this time.

\subsection{ETHANOL PRODUCT}

Fifteen days of on-site ethanol storage would be provided by the plant. The ethanol output of the plant $(4.8$ million gallons per year) will be sold to Mississippi gasoline marketers,. if a Mississippi state financial incentive is provided. If such an incentive is not provided (at the latest, in the 1983 legislative session), attempts will be made to negotiate purchase agreements in other states with sufficient tax incentives. However, these states must have open tax incentives not requiring any reciprocal agreements, and the probability of successfully competing with in-state ethanol supplies in these states is low, given the additional transportation costs involved.

\footnotetext{
* An economic analysis of a wet stillage versus dry grain
} byproduct is contained in Volume II, Appendix B. 
Currently, there is an increasing supply of ethanol in the U.S. Ethanol purchasers are now able to trade off several different sources of ethanol, which is causing ethanol prices to drop at a time when the competitive fuel price (for unleaded gasoline) is also dropping. Discussions with gasoline marketers in other states have indicated that they have no desire to commit themselves now to an ethanol purchase in the future, particularly from more remote sources where transportation costs would likely put them at a competitive disadvantage. As a result, Sun Belt has concluded that, for a plant in Mississippi, it is not possible to obtain either a volume commitment or a multiyear commitment, or even a set of agreed-upon price terms for any purchase other than one that would take place immediately. Gasoline marketers will continue to play the spot market for ethanol as long as motor fuels and ethanol are in abundant supply. This is not surprising, as it reflects good business practice on their part.

The above constraints on obtaining a purchase commitment do not exist in states with either in-state restricted tax incentives or reciprocal state tax incentives, as the supplies within these particular states may not be sufficient to satisfy the total demand of marketers in those states. In this case, it is possible to obtain a purchase commitment at least in terms of price, if not for a given volume over some period of time.

Blending on site was found to be infeasible, because each gasoline wholesaler desires a different octane product. Each needs the flexibility to blend as he wishes. Supplies of gasoline to the blenders are plentiful, since there is both a plentiful supply of crude oil to refiners and excess refining capacity available throughout the U.S.

Regarding the process efficiency and costs of varying denaturant-ethanol mixtures, a 5-percent mixture of gasoline in ethanol is optimal because (1) below 5 percent, the process uses more energy than for a benzene denaturant and (2) 5 percent is the maximum percentage of gasoline allowable and still have the gasoline sold as part of the ethanol at the ethanol price. 


\section{\begin{tabular}{ll}
\hline Ralston Purina \\
Company
\end{tabular}}

December 8, 1981

Mr. Billie E. Shepherd

President

Sun Belt Energy Corporation

212 North Main Street

Sardis, Mississippi 38666

Dear Mr. Shepherd:

This is in reply to your note of November 18 to Danny Williams concerning Ralston Purina's interest in dried distillers grains.

We would need one pound samples of the dried grains from three different days productions for inspection and analyses before we would approve the grains. If they meet our satndards, we might use them at our plants in such locations as Jackson, Mississippi; Memphis, Tennessee; or Montgomery, Alabama. The price of the dried grains in relation to other feed ingredients as well as quantities, etc., would also be involved in determining if they will be used.

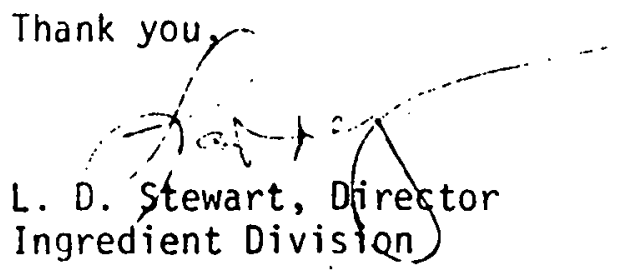

jih 
Mr. Peter G. Bos, Principal

Resource Planning. Associates, Inc.

50 Church street

Cambridge, MA

02138

Dear Mr. Bos:

Thank you for your letter of October 9, 1981. We are certainly interested in both supplying corn to the alcohol plant you described and marketing the DDG produced from the plant. Our company is actively involved in just this type of thing and works with a number of major agricultural processors in the marketing of various by-products from their processing operations. We also have arrangements with a number of facilities to supply raw material such as corn.

Our company has a full logistical operation enabling us to move the products into the best possible markets available throughout the world and thus return the highest price to the processing plant. We would like to suggest that you and/or Mr. Shepard visit with us in Minneapolis to acquaint yourselves with our operation at a time that you consider to be appropriate. We will look forward to hearing from you concerning an appropriate time to meet to pursue this matter in more detail.

If there are any questions or further information that you would like to have prior to our meeting, please do not hesistate to call upon us.

Sincerely,

I. S. JOSEPH COMPANY, INC.

Robert D. Anderson

preslaent

RDA/ung 
Exhibit 3.1 (continued)

INTERMATIONAL MULTIFOODS

1200 MULTIFOODS BUILOING

EIGHTH AND MAROUETTE

MINNEAPOLIS, MINNESOTA 55402

October 1,1981

Mr. Billie Shepard, President

Sun Belt Energy Corporation

212 N. Main

Sardis, MS 33666

Dear Mr. Shepard:

This letter is to confirm that International Multifoods, which manufactures animal feeds selling under the brand name Supersweet Feeds, considers dried distillers grains with solubles as a viable feed ingredient. Assuming reasonable price competitiveness versus other feed ingredients, dried distillers grains with solubles might be $10 \%$ to $25 \%$ of an animal feed ration. As more production becomes available, we anticipate Supersweet will be using this feed ingredient.

Assuming your project will become a reality in the future, we would welcome iurther discussions regarding dried distiller grains with solubles when you get closer to production time.

Sincerely,

INTERINATIONAL MULTIFOODS

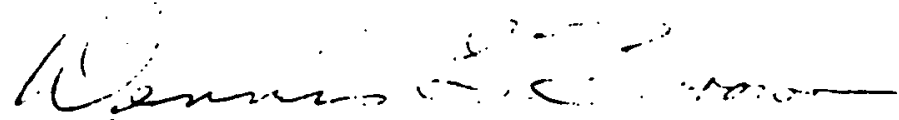

Dennis L. Brown

Directur of Procurement

DLB/mank 
Exhibit 3.2

ACRICULTURE $\cdot$ 1-H

HOME ECONOMICS

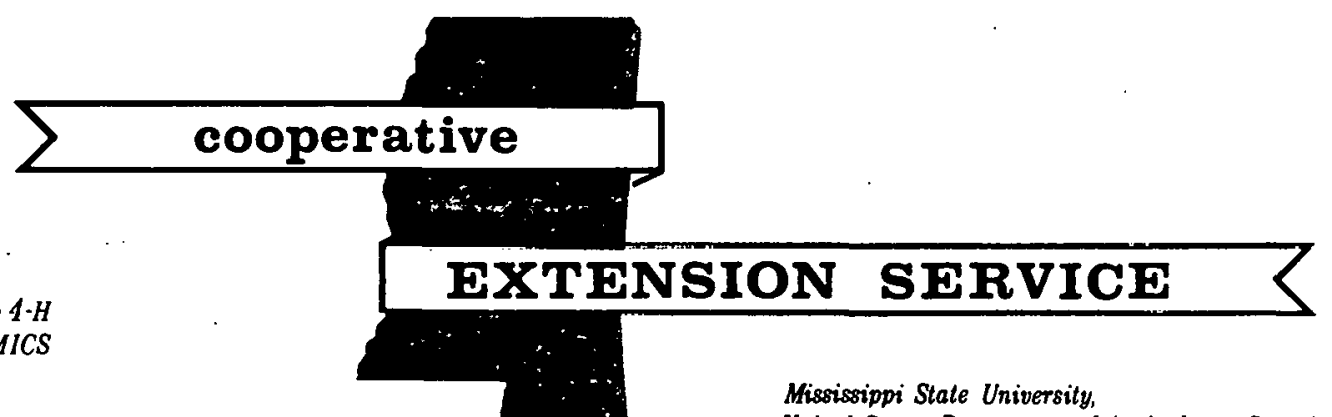

United States Department of Agriculture, Counties Cooperating

P. 0. Box 127

Sardis, Mississipp1 38666

June 1, 1982

Sunbelt Energy Corp.

Billie E. Shepard, President

212 N. Main St.

Sardis, Miss. 38666

Dear Billie:

The question has surfaced again as to the capability of Panola County to produce the needed amount of grain (corn, grain sorghum) to supply Sunbelt Energy's proposed alcohol plant. A 5 million gallon per year plant with a need of 2 million bushels of local grain sounds like an enormous task for the producers of Panola County, but lets look at the facts.

There are 306,287 acres of farm land in Panola County. Approximately twenty percent of the county lies in the Mississippi delta. The balance of the county is in the brown loam region of the State. Almost 150,000 acres are annually put into row crops in the county with the remaining land being suited for grazing and hay purposes.

In 1981, two local grain elevators bought grain sorghum for a combined total of 420,993 bushels. Farmers were pleased with this crop for two reasons: one, for the most part, these farmers who grew grain sorghum had been growing soybeans on this land; devastating erosion coupled with diseases and lowered soybean yields caused them to switch; secondly, al though the 1981 price for grain sorghum was not fantastic (around $\$ 4.00 /$ hundred wt.) producers were able to stay in the black with it. So in reality, during 1981 there was enough grain sorghum sold in Panola County to supply the plant for one-fifth of a year.

There is really no exact way of knowing how many bushels of grain sorghum were turned away at these two local grain elevators. We know from personally talking with many farmers who produced grain sorghum that getting the elevators to buy it was a job in itself.

Sorghum with even traces of foreign matter or a percentage point high or low on moisture was not bought. A conservative estimate for Panola County would be that as much sorghum was held and fed to livestock as was sold in 1981. So the total grain sorghum yield for Panola County in 1981 was approximately 842,000 bushels, 42 percent of what is needed for the Sunbelt Energy Plant. 
Page 2

Speaking as a county worker for the Mississippi Cooperative Extension Service, I am totally convinced that Panola County can grow an abundance of corn and grain sorghum. Panola County farmers want and need to grow corn and grain sorghum. There is only one problem - no avallable market. American farmers are suffering under the worst set of economic conditions since the Great Depression. A farmer cannot make a living today and frankly, I would like to see something done about it. Creating a market in Panola County for corn and grain sorghum would answer many a man's prayers. If the money was in it for the farmer, much of the 150,000 acres of available cropland in Panola County would be planted to corn and grain sorghum.

In conclusion, to answer the initial question of the capability of Panola County to produce the needed amount of grain to supply Sunbelt Energy's proposed alcohol plant, I would answer with a resounding yes. To try and determine the amount of each that would be grown in any given year would be pure speculation. However, I will say that even in a bad year, enough grain sorghum alone could be grown in Panola County to supply the plant. For example, trials in Mississippi during 1980, al though a terrible year for production, produced average grain sorghum yields of about 50 bushels per acre. At this output, 40,000 acres would need to be planted to produce $2,000,000$ bushels; and this is during a bad year.

I hope this information and my opinions will help you in your ongoing plight to complete the alcohol plant. If I can be of any further help, p.lease call.

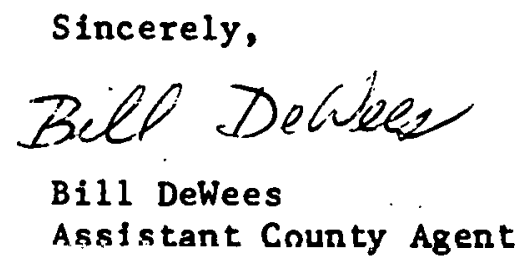

$\mathrm{BD} / \mathrm{bbm}$ 
Exhibit 3.3

OPTION TO PURCHASE GRAIN

THIS OPTION IS TAKEN BY SUN BELT ENERGY CORPORATION FOR FUTURE ASSIGNMENT TO THE COMPANY THAT WILL COMMIT ITSELF TO BUILD AND OPERATE BOILERS UTILIZING BIOMASS AND OTHER FORMS OF ENERGY TO MANUFACTURE PROCESS STEAM TO BE UTILIZED PRIMARILY IN THE MANUFACTURE OF ALCOHOL FROM GRAINS.

1. PARTIES: The parties to this agreement are

hereinafter referred to as "Owner(s)", and Sun Belt Energy

Corporation, a Mississippi corporation, hereinafter referred to as "SBEC" of 212 North Main Street, Sardis, Mississippi.

2. CONSIDERATION: The consideration for the granting of this option is the benefit to owner(s) of the continuing efforts of SBEC toward the establishment of a plant utilizing biomass and other forms of energy to manufacture process steam utilized primarily for the manufacture of alcohol from grain in Panola County, Mississippi.

3. OPTION: Owner(s) hereby grant(s) unto SBEC an exelusive option to purchase Owner(s) entire production output of to be grown during the 1983-1984 crop year on the following described property, to-wit:

4. TERM: The term of this option shall extend from the date hereof until 12:00 O'clock midnight CST, on . 1983 .

5. RENEWALS AND EXTENSIONS: In the event that SBEC shall exercise its rights under this Option, or any renewal or extension thereof, prior to its expiration, then and in that event, this Option shall automatically be renewed and extended from the date of such exercise until 12:00 $0^{\prime} \mathrm{clock}$ midnight, CST, on the 365 th day therefrom, and such automatic renewal or extension shall cover and effect the next succeeding crop year, except that nothing contained herein shall be construed to renew or extend this option past the 1993-1994 crop year.

6. EXERCISE: This Option, or any extension or renewal thereof, may only be exercised by SBEC's giving written notice to Owner of SBEC's intention to exercise the Option, extension or renewal, said notice to be deposited, postage prepaid with the United States Mail, before the expiration of the term of the Option.

7. NOTICE: All notice required by this Option shall be sent to the address of the appropriate party as set out in Number 1 above.

8. EXPIRATION: In the event that SBEC fails to exercise this Option, extension or renewal, according to the terms and conditions hereof, then and in that event, this option, extension or renewal shall become null, void and of no legal effect.

9. PRICE: The price to be pald for purchased under the terms of this option, or any renewal or extension thereof, shall be the Chicago Board of Trade closing price for on the date of the exercise of this option, or any renewal or extension thereof, said price being F.O.B. SBEC plant site., wherever said plant site shall be located in Panola County. Mississippi.

10. PAYMENT: SBEC shall make payments to Owner(s) on a weekly basis as the is delivered to SBEC's plant site. SBEC shall furnish to owner(s) at time of payment, a statement setting out the manner in which the payments were calculated. All payments under this option, or any extensions or renewals thereof, shall be made prior to expiration.

11. OTHER DOCUMENTS: Owner shall execute such other documents and instruments as are necessary to effectuate the purpose of this agreement. 
Exhibit 3.3 (continued)

12. DUTIES OF OWNER(S): Upon the exercise of this Option, or any extension or renewal thereof, the owner(s) shall plant, fertilize, cultivate and otherwise grow the sold hereunder in a good husbandlike manner and shall supply at his own cost and expense all labor, water, fertilizer, chemicals and other material necessary for the proper cultivation and growth of the

It shall further be the duty of the Owner(s) to harvest and store said upon its reaching a mature stage, and to deliver same to SBEC at such times and in such amounts as SBEC may require from time to time, all to be don at owner(s) cost and expense.

13. DISPUTES: In the event that disputes arise under this Option, or any renewal or extension thereof, between owner(s) and SBEC regarding any of the terms hereof, and same cannot be mutually resolved within

days, then and in that event, said disputes shall be refer $\overline{\text { red to }}$ arbitration and decision by three (3) arbitrators, one of whom shall be appointed by Owner (s) and one of whom shall be appointed by SBEC, and two (2) arbitrators so appointed shall appoint a third arbitrator by agreement between themselves. The three (3) arbitrators so appointed shall reach a decision as to the dispute and shall submit same to owner and to SBEC in writing. The decision of the arbitrators shall be final as between Owner(s) and SBEC.

14. ASSIGNMENT: SBEC as used herein refers to Sun Belt Energy Corporation, its successors or assigns. SBEC covenants and agrees that it shall make no assignment of this option and conditional conveyance other than to an entity which shall, subseguent to the date hereof: (a) enter into a contract for the construction of a plant for the utilization of biomass and other forms of energy for the manufacture of alcohol in Panola County, Mississippi, or (b) enter into a contract for the operation of steam boilers in connection with such a plant, or (c) enter into a contract to supply grain for the production of alcohol at such plant. Any assignment in violation of this restriction, whether by voluntary or involuntary means, shall be utterly void and of no legal effect.

OWNER(S), BY HIS (THEIR) SIGNATURE(S) HEREON, COVENANTS: THAT HE HAS READ AND UNDERSTANDS THE ENTIRE FOREGOING OPTION TO PURCHASE GRAIN; THAT THIS AGREEMENT CONTAINS THE ENTIRE AGREEMENT BETWEEN OWNER(S) AND SBEC; AND THAT OWNER, HIS HEIRS, SUCCESSORS AND ASSIGNS SHALL BE BOUND BY THE TERMS HEREOF.

EXECUTED this the

WITNESS : day of 198
OWNER

OWNER'S ADDRESS 
THIS OPTION IS TAKEN BY SUN BELT ENERGY CORPORATION FOR FUTURE ASSIGNMENT TO THE COMPANY THAT WILL COMMIT ITSELF TO BUILD AND OPERATE BOILERS UTILIZING BIOMASS AND OTHER FORMS OF ENERGY TO MANUFACTURE PROCESS STEAM TO BE UTILIZED PRIMARILY IN THE MANUFACTURE OF ALCOHOL.

\section{PARTIES: The parties to this agreement are}

hereinafter referred to as "Owner(s), and Sun Belt Energy Corporation, a Mississippi corporation, hereinafter referred to as "SBEC" of 212 North Main street, Sardis, Mississippi.

2. CONSIDERATION: The consideration for the granting of this option is the benefit to Owner(s) of the continuing efforts of SBEC toward the establishment of a plant utilizing biomass and other forms of energy to manufacture process steam utilized primarily for the manufacture of aicohol in Panola County, Mississippi.

3. OPTION: Owner(s) hereby grants to SBEC an exclusive option to purchase and conditional conveyance of certain wood and wood products, subject to existing liens thereon, located in to-wit:

Approximately tons of gualified wood and/or wood products, being approximately wood and/or wood products/epproximately percent of the qualified gualified wood and/or wood products, standing, lying, growing, being produced or otherwise situated on the following described property owned by Owner(s), to-wit:

4. QUALIFIED WOOD AND/OR WOOD PRODUCTS: Qualified wood and/or wood products shall be defined for the purposes of this option as including:

Those types and species of forestry products not ordinarily used in the lumber or pulpwood industry, commonly referred to as "scrubby hardwoods" located primarily on ridgetops and upslopes; and those types of by-products from the timber, lumber and/or pulpwood business including, but not limited to, sawdust, shavings, bark and tops.

5. TERM: The term of this Option shall extend from the date hereof until 12:00 0 clock midnight, CST on February 28, 1983. In the event that this option is not exercised according to the conditions and agreements set forth herein, then this option shall become null, void and of no legal effect.

6. EXERCISE: This Option may only be exercised by SBEC's giving written notice to Owner(s) of SBEC's intention to exercise the option, said notice to be deposited, postage prepaid with the United States Mail, before the expiration of the term of the Option.

7. NOTICE: All notice required by this option shall be sent to the adiress of the appropriate party as cot out in Number 1 above.

8. PRICE: It is understood and agreed by both Owner(s) and SBEC that the gualified wood and/or wood products which are the subject of this option have traditionally been unsaleable and that no market price now exists for same. It is further agreed and understood that the price to be paid to Owner(s) by SBEC in the event that SBEC exercises his option shall be the Mississippi market price on a per ton basis for that gualified wood and/or wood products harvested or produced from the premises described in Number 2 above, on the date that the option is exercised. In the event that no Mississippi market price exists on the date that the option is exercised, ther price shall be as then agreed upon by Owner(s) and SBEC. In the event that no Mississippi market price exists, as said above, and in the further 
Exhibit 3.4 (continued)

event that no agreement can be arrived at by Owner(s) and SEEC within ten (10) days of the exercise of the option, then the issue of the price on a per ton basis shall be referred to arbitration and decision by three (3) arbitrators, one of whom shall be appointed by Owner(s) and one of whom shall be appointed by SBEC, and the two (2) arbitrators so appointed shall appoint a third arbitrator by agreement between themselves. The three (3) arbitrators so appointed shall reach a decision as to contract price on a per ton basis and shall submit same to Owner(s) and to SBEC in writing. The decision of the arbitrators shall be final as between Owner(s) and SBEC.

9. PAYMENT: SBEC shall make payments to Owner(s) on a weekly basis as the qualified wood or wood products are harvested and/or transported. SBEC shall furnish Owner(s), at the time of payment, with a statement setting out the manner in which the payments were calculated. All weights shall be determined by SBEC at its future plant site. All payments under this Option shall be made prior to 12:00 $0^{\prime c}$ clock midnight, CST February 28, 1993.

11. MODIFICATION AND RECISION: This Option shall automatically be modified and rescinded in part without further action of Owner(s) or SBEC as it applies to the conveyance of that part of the qualified wood and/or wood products which may suffer loss, damage or deterioration prior to harvest and as a result does not meet the needs and requirements of SBEC.

12. OTHER DOCUMENTS: Owner(s) shall execute such other documents and instruments as are necessary to effectuate the purpose of this agreement.

13. HARVEST AND/OR TRANSPORTATION: SBEC shall consult with Owner(s) concerning harvest and/or transportation of the gualified wood and/or wood products and SBEC shall make good faith effort to conform to owner(s) objectives. The decision of SBEC regarding the time and manner of harvest and/or transportation shall control.

14. EXPIRATION OF CONVIYANCE: All rights of SBEC arising upon the exercise of this Option shall cease and expire at 12:00 o'clock mianight, CST, February 28, 1993.

15. ASSIGNMENT: SBLC as used herein refers to Sun Belt Energy Corporation, its successors or assigns. SBEC covenants and agrees that it shall make no assignment of this option and conditional conveyance other than to an entity which shall, subseguent to the date hereof: (a) enter into a contract for the construction of a plant for the utilization of biomass and other forms of energy for the manufacture of alcohol in panola County, Mississippi, or (b) enter into a contract for the operation of steam boilers in connection with such a plant, or (c) enter into a contract to supply gualified wood and/or wood products for the operation of such steam boilers at such plant. Any acoignment in vlularion of this restriction, whether by voluntary or involuntary means, shall be utterly void and of no legal effect.

OWNER (S) BY

SIGNATURE HEREON, COVENANTS: THAT

HAS READ

AND UNDERSTANDS THE ENTIPE FORFGOING OPTION TO PURCHASE WOOD ĀND/OR WOOD PRODUCTS: THAT THIS AGRLEMENT CONTAINS THE ENTIRE AGREEMENT BETWEEN OWNER (S) AND SBEC: AND THAT OWNER(S) SHALL BE BOUND BY THE TERMS HEREOF. HEIRS, SUCCESSORS AND ASSIGNS

EXECUTED this the WITNESS : day of 198

\section{OWNER (S)}

OWNER'S ADDRESS 
Exhibit 3.4 (continued)

STATE OF MISSISSIPPI

COUNTY OF

PERSONALLY appeared before me, the undersigned authority in and

for the jurisdiction aforesaid,

who acknowledged that

signed and delivered the faregoing instrument

on the day and in the month and year thereof as

free and voluntary act and deed.

GIVEN under my hand and official seal of office on this the day of 198

(SEAL)

My Commission expires: 


\section{Wood Services inc. \\ P.O. Box 314 \\ Sardis, Mlssissippl 38668 \\ Phone 601-487-3024}

March 30, 1982

Bill Shepard

Sun Belt Energy Corp.

212 North Main Street

Sardis, Miss. 38666

Dear Bill:

Enclosed is the reduced study for the 120 ton per day requirements of wood waste boilers. 938 of all waste is in saw dust or chip form. The remaining 58 to 88 is in slabs or round wood.

As stated earlier, the production of the wood products industry is as low as it has been in history. The 508 level of production at this time is still sufficient to supply our needs. The additional development of the round wood production for our needs will keep the requirement at a very comfortable point.

As you can see, there is a wide range of pricing form some points. There is a market to some extent in the state. The average market price for saw dust and chips not used in paper or press board is $\$ 15.00$ to $\$ 20.00$ per ton delivered. This is what we will have to compete with.

The development of round wood is a question at this time, at the price we are trying to work with. With wood at no cost it is possible, but if the contractor has to purchase the scrub timber it is not possible to develop large volumn and kcep the production sound. You need to think in terms of $\$ 12.00$ to $\$ 15.00$ per ton delivered, where we have to. We now have several large producers of waste at about $\$ 3.00$ out of our range.

If you need any additional information, please give me a call.

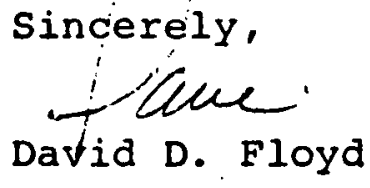

Encl.

$\mathrm{DDF}: \mathrm{mm}$ 
WOOD SUPPLY STUDY

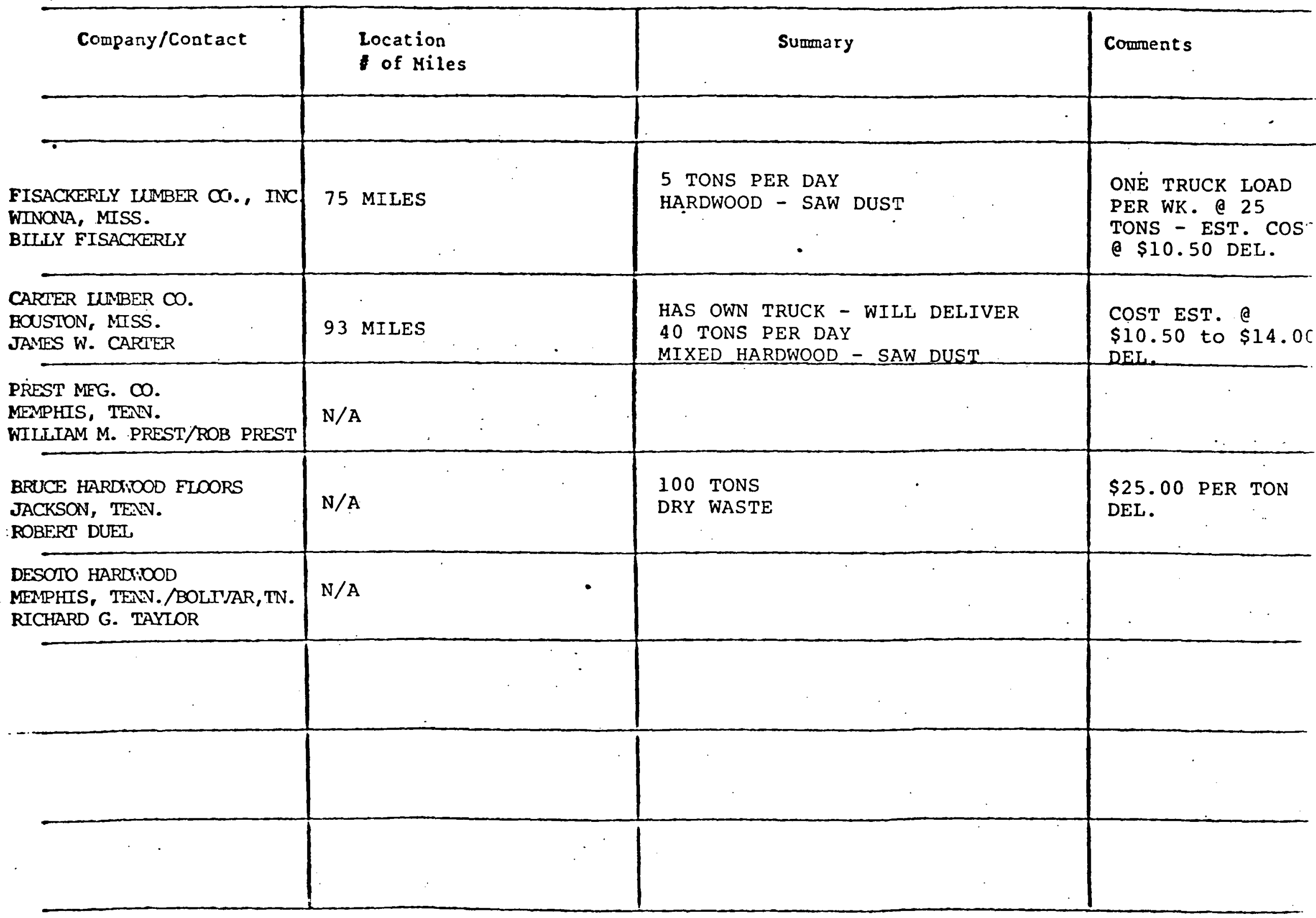




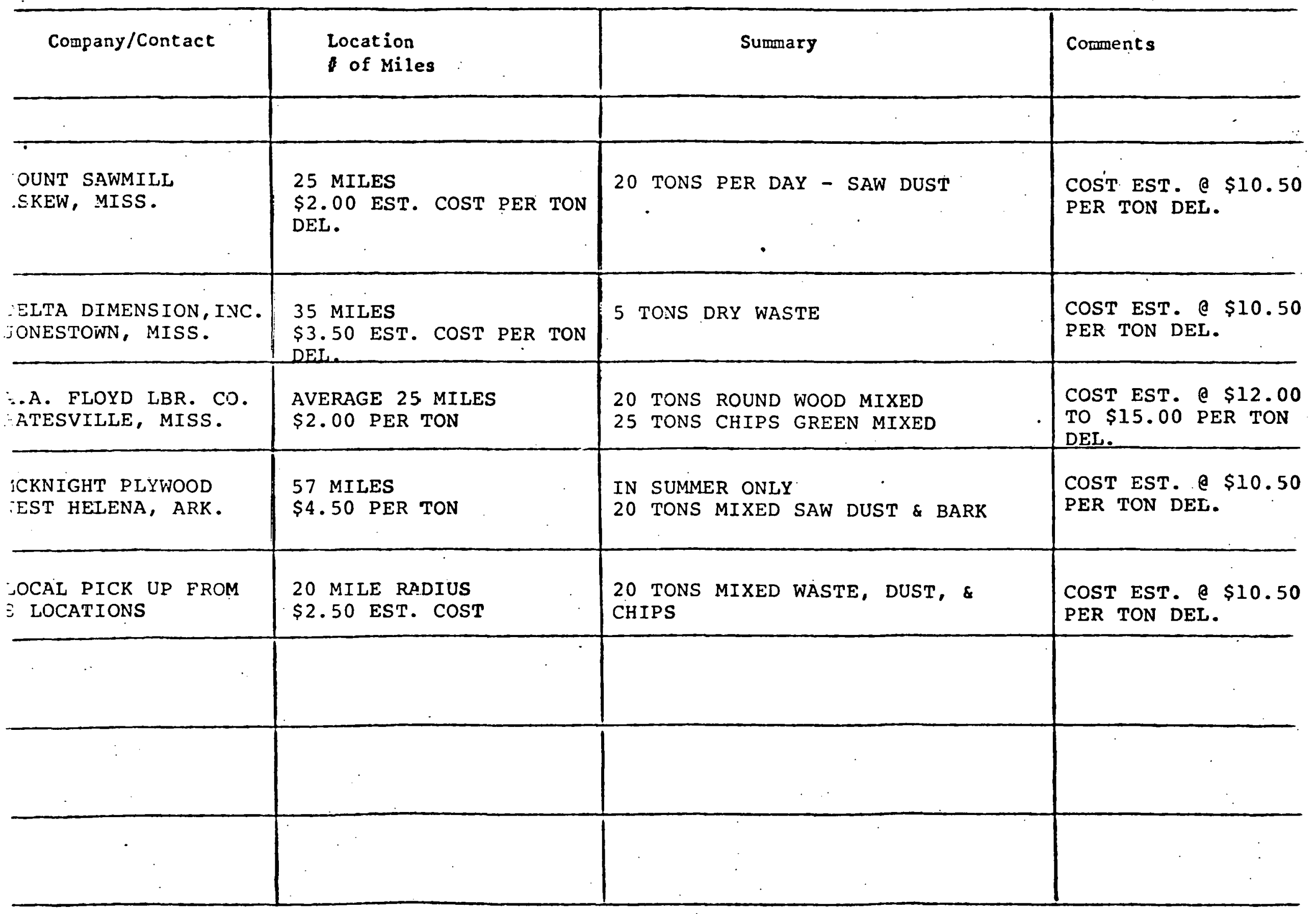


SEITZ LUMBER CO., INC. WEST POINT, MISS. ED SEITZ, JR.

TALLAHATCHIE HARDWOODS, INC CHARLESTO:, MIISS.

HERMAN YOJAG LUMBER $\infty$. FORREST CITY, ARKANSAS HERMAN YOING

MEMPHIS FURVITURE MFG. $\infty$. NEMPHIS, TENV.

JAMES W. LOVING

CHARLFS O. COX CORP. NEMPIIS, TEIV. STANLEY H. WANZER MEMPHUS FOIDING STAIRS NEMPHIS, TENN. JOHN STUCKER

LINDEN LUTBER CO. ITNDEN, PLABAMA DON OVERMIER/JACK KETLY

GRIFFITH ILIRER $\infty$. MADISON, ARKANSAS GRAHAM GPIFFITH

\section{MILES}

\section{MILES}

EST. COST PER TON

$\$ 6.61$
Comments

COST EST. Q

HAS OWN TRUCKS - WILL DELIVER

35 TONS OF MIXED HARDWOOD SAWDUST

40 TONS OF MIXED HARDWOOD ,

SAW DUST \& SLAB
$\$ 10.50$ DEL.

COST EST.

$\$ 13.00$ to $\$ 15.00$ PER TON DEL.

\begin{tabular}{|l|l|l} 
& & \\
\hline & & \\
\hline $\begin{array}{l}\text { N/A MILES } \\
\$ 4.50 \text { EST PER TON }\end{array}$ & 3.6 TONS PER DAY & $\begin{array}{l}\text { COST EST. Q } \\
\text { \$10.50 PER TON D: }\end{array}$ \\
\hline
\end{tabular}




\begin{tabular}{|c|c|c|c|}
\hline Company/Contact & $\begin{array}{l}\text { Location } \\
\text { of Miles }\end{array}$ & Summary & Comments \\
\hline & & & - \\
\hline $\begin{array}{l}\text { PEITZ PLYWDOD CORP. } \\
\text { PEPHIS, TEN. } \\
\text { I. J. BARREIT }\end{array}$ & $\begin{array}{l}55 \text { MILES } \\
\text { EST. COST @ } \$ 4.50 \text { PER } \\
\text { TON }\end{array}$ & 4 TONS DRY WASTE & $\begin{array}{l}\text { COST EST. } \\
\$ 10.50 \text { TON DEL. }\end{array}$ \\
\hline $\begin{array}{l}\text { AUST BAND SAN MULL, INC. } \\
\text { EST HEIENA, ARKANSAS } \\
\text { HOMAS E. FAUST }\end{array}$ & $\begin{array}{l}57 \text { MILES } \\
\text { EST. COST @ } \$ 4.45 \text { PER } \\
\text { TON }\end{array}$ & $\begin{array}{l}\text { HAS OWN TRUCK } \\
5 \text { TONS SUMMER ONLY }\end{array}$ & $\begin{array}{l}\text { COST EST. }{ }^{a} \\
\$ 10.50 \text { PER TON } \\
\text { DEL. }\end{array}$ \\
\hline $\begin{array}{l}\text { IVES \& REYNOLDS LBR. CO. } \\
\text { UISVILIE, MISS. } \\
\text { ERRY REYINIDS }\end{array}$ & $\begin{array}{l}140 \text { MILES } \\
\text { EST. COST \& } \$ 9.80 \text { PER } \\
\text { TON }\end{array}$ & $\begin{array}{l}70 \text { TONS PER DAY } \\
\text { SAW DUST \& BARK }\end{array}$ & $\begin{array}{l}\text { COST EST. a } \\
\$ 15.00 \text { PER TON } \\
\text { DEL. }\end{array}$ \\
\hline $\begin{array}{l}\text { UTORIAN CORP. } \\
\text { UPORA, MISS. } \\
\text { TEVE CARROLL }\end{array}$ & $\mathrm{N} / \mathrm{A}$ & 25 TONS DRY WASTE & $\begin{array}{l}\text { COST EST. @ } \\
\$ 25.00 \text { PER TON } \\
\text { DEL. }\end{array}$ \\
\hline $\begin{array}{l}\text { IDGELYY SATWIILIL } \infty . \\
\text { IDGELY, TENV. } \\
\text { ILKES NOORE, JR. }\end{array}$ & $\begin{array}{l}\text { N/A } \\
\text { OUT OF BUSINESS }\end{array}$ & & , \\
\hline $\begin{array}{l}\text { ILSON LURRER CO. } \\
\text { EMPHIS, TEMN. } \\
\text { HARLES. F. WIISON }\end{array}$ & $\mathrm{N} / \mathrm{A}$ & & \\
\hline $\begin{array}{l}\text { NDERSON-TULEY COMPANY } \\
\text { ICKSBURG, MISS. } \\
\text { ARKER HAIU }\end{array}$ & N/A & 100 TONS MIXED WASTE & $\begin{array}{l}\text { COST EST. @ } \\
\$ 25.00 \text { PER TON } \\
\text { DEL. }\end{array}$ \\
\hline $\begin{array}{l}\text { ORTON MFG. CO. } \\
\text { EMPHIS, TENLN. } \\
\text { OHN D. WILSON, JR. }\end{array}$ & $\begin{array}{l}55 \text { MILES } \\
\text { EST. COST \& } \$ 4.50 \text { PER TO } \\
\text { FRT. }\end{array}$ & $\begin{array}{l}20 \text { TONS OF SAW DUST } \\
\text { MIXED HARDWOOD }\end{array}$ & $\begin{array}{l}\text { COST EST. O } \\
\$ 10.50 \text { PER TON } \\
\text { DEL. }\end{array}$ \\
\hline
\end{tabular}


Without a state financial incentive, a grain-to-alcoholfuels project is not economically and financially feasible in Mississippi. Even if the federal gasoline excise tax exemption remains, the ethanol could only be sold at a price of $\$ 1.25$ - $\$ 1.35$ per gallon, a price well below that required to provide sufficient debt coverage and an acceptable return on equity. As a result, the only possibility -- and it is a slight one -- for a Mississippibased plant to be economical is to find an ethanol market in a state with at least a $4 \not k$ per gallon tax incentive. Such an incentive, coupled with a clearly stated federal policy to retain the $4 \not C$ federal excise tax exemption, would provide sufficiently attractive economics.

On this basis the analysis that follows assumes a $4 \not$ tax incentive in a state where the ethanol is sold, and a $4 \notin$ federal excise tax incentive, and examines sensitivities in financial performance to changes in key assumptions for the project.

\subsection{PROJECT COST ESTIMATES AND A.SSUMPTITONS*}

The estimated maximum plant cost is $\$ 15.5$ million, as shown in Exhibit 4.1. Construction interest is estimated at $\$ 2.5 \mathrm{million}$. Land acquisition and rights ( $\$ .3 \mathrm{mil}$ ), financing costs $(\$ .2 \mathrm{mil})$, the process fee $(\$ .4 \mathrm{mil})$, and detailed resource and market assessment ( $\$ .1 \mathrm{mil}$ ) are estimated to be $\$ 1$ million, and working capital required at plant start-up an additional $\$ 2$ million, for a total project capital cost requirement of $\$ 21 \mathrm{million}$. The annual operating cost estimates are shown in Exhibit 4.1, second page.

* Details of the plant cost estimates are included in Volume II. 
Sun Belt intends to guarantee the reliability of the capital-cost projection by negotiating a fixed-price turnkey construction contract. This contract will

include a retainage clause, which will allow sun Belt to withhold 10 percent of the agreed-upon construction fees until project completion.

\subsection{CASH FLOW/PROFITABILITY}

The projected discounted cash flow return on total investment of the project is estimated at about 20 percent, the return on equity with 75-percent debt at about 25-30 percent, and the debt coverage ratio* at about $2: 1$ in the first year of operation, increasing thereafter. Employing a sale and leaseback or similar financing structure to use the tax benefits more effectively, the return on net equity would exceed 50 percent. These projections are based on the following key assumptions:

- Capital costs (installed dollars):

$\$ 15.5$ million

- Ethanol sales price (1982

dollars, f.o.b. plant):

1.70. per gallon**

- Net ethanol cost to blender

(1982 dollars):

$\$ 0.90$ per gallon

- DDG sales price (1982 dollars):

$\$ 170.00$ per ton

- Corn cost (1982 dollars):

$\$ 2.30$ per bushel

- wood cost (1982 dollars):

$\$ 10.70$ per ton

\footnotetext{
* Operating cash flow divided by the debt service requirement.

** Based on a $4 \not$ per gallon exemption for alcohol blended fuels from the federal gasoline excise tax, and a $4 \not \subset$ per gallon tax exemption in the state where the alcohol fuel is sold.
} 
Exhibits 4.2 through 4.5 present a summary of the assumptions and financial results for the project, and the projected income/cash flow statement, balance sheet, and sources and uses of funds statement for the project. Under the assumptions used, the project would pay back within 3 years of the start of operations.

\subsection{RISK AND SENSITIVITY}

For alcohol fuels there is currently an exemption from the $4 \not / g a l l o n$ federal gasoline excise tax, and a special 10 percent energy tax credit. With at least a $4 \not / g a l i o n$ state tax exemption, the project cash flow would be slightly positive (after debt service) without these federal incentives.

The debt service coverage under a range of possible adverse price and cost developments is important to the Einancing of the project. Accordingly, sun Belt has carried out a range of sensitivity analyses of gasoline and corn prices in terms of their impact on the debt coverage and the return on equity. In Exhibit 4.6 these sensitivities are shown for a range of ethanol sales prices and corn purchase prices. Note that while the debt coverage ratios are for a given year, the after-tax return on equity (ROE) represents the entire stream of cash flows over the operating life of the project. Hence, the cash flow in any given year may not be indicative of the overall return on equity.

Further sensitivities of the debt coverage in the first year are shown with respect to the cost of wood, inflation rate, interest rate, operating output level, elimination of federal tax incentives, and combinations of various factors that could produce negative impacts. These downside risk analyses are compared to the base case that is reflected in Exhibits $4.2-4.5$.

For reference, Exhibit 4.8 shows historical price trends of various grains and gasoline. Further, Appendix B contains the computer model result of the sensitivity analyses shown in Exhibits 4.6 and 4.7 . 


\section{4 FURTHER FINANCIAL CONSIDERATIONS}

Several factors could improve the financial performance of the project. Each of these is briefly

described qualitatively below.

- Interest costs lower than 16 percent would increase the ROI.

- A drop in corn prices below the existing price would increase the ROI.

- Use of grains other than corn, at those times when their price was more economically attractive, would increase the ROI. (Downside sensitivities for corn price were analyzed in the previous section.)

- Use of lower-priced distressed grain as part of the feedstock supply could increase the ROI.

- Development of a wet stillage market through local cattle feedlots would eliminate the need for grain drying, thus reducing energy costs and increasing the ROI.

- A tightening by the U.S. Environmental Protection Agency of refiner lead content standards would increase the attractiveness of ethanol as an octane enhancer, which would increase the demand and price for ethanol and enhance the project's profitability.

- An above-inflation increase in oil, gasoline, and related ethanol prices would increase the ROI. (Exhibit 4.6 shows the downside risk.)

- Operation of the plant at higher than rated capacity, a capability already demonstrated in other plants, would increase the ROI.

- Operation above 333 days per year would increase the ROI.

- Lower wood fuel costs would increase the ROI.

- Start-up of the plant earlier than December 31, 1984, would produce revenues in 1984 (not assumed in the financial analysis), and would thus increase the project ROI.

- Start-up of the plant later than December 31, 1984, would increasc construction interest and delay the initial revenues, which would decrease the ROI. However, these effects would be mitigated by qualification for a more attractive depreciation schedule (effective January 1, 1985) under the 1981 Economic Recovery Tax Act. 


\subsection{PROJECT FINANCING PLAN}

Discussions were held with commercial banks, insurance companies, pension fund managers, and potential equity participants. Complete financing interest has been obtained, subject to passage of a Mississippi state tax incentive. *

While the specific project financing plan must be developed in close collaboration with the debt financing and equity participants, the following considerations are presented, based on the financial structure of the sun Belt Energy Corporation and previous financing experience with plants of this type:

- The fixed plant costs can be financed up to 75 percent through debt financing by an institution with funds available for long-term commitment (e.g., insurance company, pension fund, trust fund). The fixed plant costs are estimated at $\$ 19$ million, which includes the plant capital costs ( $\$ 15.5$ million), construction interest ( $\$ 2.5 \mathrm{million})$, and cost of land acquisition and rights $(\$ 1.0 \mathrm{million}) . * \star$

- The $\$ 2$ million working capital costs can be largely funded by a commercial bank line of credit, secured by the accounts receivable.

- The ability to fund current assets through accounts payable and other current liabilities is limited, since it is the practice with this type of plant to pay upon receipt of the grain feedstock and wood fuel.

- The portion of the fixed plant costs and working capital not financed by debt financing sources must be supplied by equity participants. These would likely fall into two cateqories:

- Equity participants not involved in the management of the plant, whose equity investment would be related mainly to the investment tax credit $(\$ 1.6$

* The names of the financing parties cannot be supplied for confidentiality reasons.

** Federal funding or guarantees were found to be unnecessary if a $7 \not / g a l i o n$ state tax incentive was provided. Without a state tax incentive, the plan would not be economically viable enough to meet federal funding requirements. Also, the availability of federal funding support continues to be uncertain. 
million available the first year of operation) and the equipment depreciation benefits ( $\$ 16 \mathrm{million}$ in tax deductions over the first 5 years of operation). Since the Sun Belt Energy Corporation has no other operating and taxable income, the financing structure must provide for the use of these tax benefits by another equity participant.

- Equity owners with a direct interest in the management and operation of the plant.

- A construction loan to cover the fixed plant costs can be acquired from short-term financing sources (e.g., commercial bank) or longer-term funding sources, contingent on permanent financing to take out the construction loan at the time of plant start-up.

The following guarantees and assurances will be available to the debt financers and equity participants.

- The construction and start-up of the plant will be completed under a fixed-price, turnkey contract with the general contractor and design firm

- The design firm will warrant the total production capacity and technical performance of the plant

(a) for the grain:

- gallons ethanol/bushel and lbs DDGS/bushel

(b) for the plant:

- total steam consumption

- quantity and quality of ethanol and DDGS \& $\mathrm{CO}_{2}$

- total electricity use

- total water use

- total chemical use

- The general contractor will be bonded

- Non-completion and business interruption insurance will be purchased. 
Exhibit 4.1

Sun Belt Ethanol Plant Capital Cost Summary (thousands of dollars)

Item

Cost

Equipment

$\$ 5,450$

Instruments

770

Piping

970

steel

550

Insulation

Electrical 350

Concrete 480

Buildings 230

Site Improvements 500

Painting 40

Construction Indirects

110

\begin{tabular}{|c|c|c|c|}
\hline & Subtotal & 99,530 & $\$ 9,530$ \\
\hline Engineering, $i$ & including burdens & & $\$ 1,720$ \\
\hline $\begin{array}{l}\text { Procurement, } \\
\text { technical serv } \\
\text { burden) }\end{array}$ & $\begin{array}{l}\text { administration, } \\
\text { vices (including }\end{array}$ & & 480 \\
\hline Construction $\pi$ & management & & 300 \\
\hline \multirow{3}{*}{$\begin{array}{l}\text { Full service } \\
\text { Construction }\end{array}$} & engineering contractor's & fee & 450 \\
\hline & contingency & & 1,690 \\
\hline & Subtotal & dollars) & $\$ 14,170$ \\
\hline \multicolumn{3}{|c|}{ Inflation contingency } & 1,330 \\
\hline & Total & (alled). & $\$ 15,500$ \\
\hline
\end{tabular}


Exhibit 4.1 (continued)

Ethanol Plant Annual Operating Cost Summary

Item

Annual Cost (million 1982 dollars)

Variable costs

Grain feedstock

$4 \cdot 4^{a}$

Fuel

.4

Electric power

.3

Drying agent

.3

Other (e.g., supplier,

.6

chemicals)

Fixed Costs

Labor

.75

$\mathrm{G} \& A$

.3

Depreciation

$2.5-3.6^{b}$

Interest

$.3-2.1^{c}$

a. Assuming a corn price of $\$ 2.30$ per bushel.

b. Varies in the first 5 years according to the equipment depreciation schedule.

c. Varies during the debt repayment period. 


\section{Exhibit 4.2}

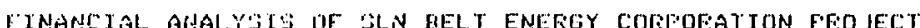

CORN MOMETHANDL FLANT

4.8 MIL GFY RATEL CAFACITY

ASSIJRFTIUNS ( 1982 \$)

14209 GFD RATEU CAP

$100 \%$ ACT CAP
DATE OF ANALYSIS: $14,104 / 82$

FUEL: wOON

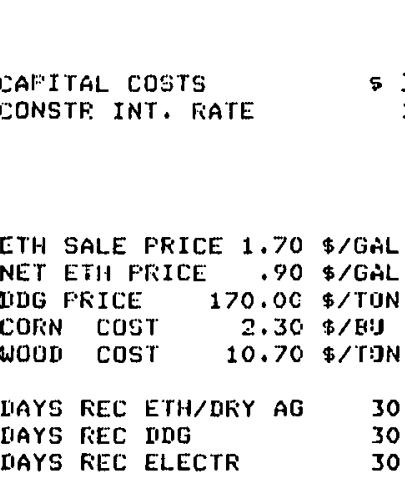

$14.2 \mathrm{MIL}$
$16.0 \%$
IL
OL
OJ
30
30
30

$\begin{array}{ll}\text { PERCENT LT DEST } & 75 \% \\ \text { LT DEET INT FIATE } & 16.0 \% \\ \text { LT UERT VIEFAY } & 10 \% \text { YFIS } \\ \text { BANK LINE INT FT } & 16.0 \%\end{array}$

$\begin{array}{ll} & \\ \text { ELECTK FURCH COST } & 5.0 \mathrm{c} / \mathrm{KWH} \\ & \\ \text { DAYS FAY COFN } & 0 \\ \text { IAYS FAY WOOD } & 0\end{array}$

\begin{tabular}{l} 
INFLATIUN FATE \\
CAF CQST FEEAL ESC \\
\hline $.0 \%$
\end{tabular}

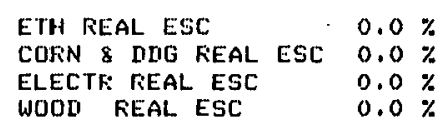

LIFO INUENTORY POLICY

I5 DAYS ETH STOFIAGE

15 DAYS CORIN STORAGE
FEN INC TAX
STATE INC TAX
STATE SALES IAX EXEMFT
STATE GASOLINE EXCISE TAX EXEMFT
FED GASOLINE EXCISE TAX EXEMFT

LAST YF OF STATE TAX EXEMFT

\section{$46.0 \%$ \\ $4.0 \%$ \\ 0.0 C/GAL
4.0 C/GAL \\ 4.0 C/GAL \\ $4.0 \mathrm{C} / \mathrm{GAL}$
1999}

\section{EQUIF' DEFR LIFE EQUIF ITC} ELLG ITC

5 YRS

$0 \%$

RESULTS

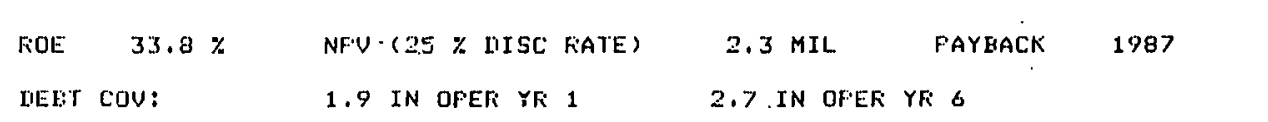

ENG COSTS \$ .6 MIL INITIAL IEET $\$ 12.4 \mathrm{mIL}$

MAXIMUM CASH ERUITY \$7.6 MIL

ANNUAL SALES:

A.8 MIL GALS ETH

16649 rONS InG

$0.0 \mathrm{MW} \mathrm{LEECT}$

ANRUAL FLAANT MELES

$1.9 \mathrm{MIL}$ BU CORN

6 MIL KWH

29273 ACRES CORH

166 MIL LII STEAM

16\&49 HEAN CATTLE

13 LES DIL/BU 


\section{Exhibit 4.3}

\begin{tabular}{|c|c|c|c|c|c|c|c|c|c|}
\hline REVENUE: & $19: 82$ & $\llbracket 983$ & 1984 & 1985 & 1986 & 1987 & 1908 & 1909 & 1990 \\
\hline ETHAMOL & 0.0 & 0.0 & 0.0 & 10.2 & 11.0 & 11.9 & 12.8 & 13.9 & 15.0 \\
\hline IIng & 0.0 & 0.0 & 0.0 & 3.6 & 3.9 & 4.2 & 4.5 & 4.9 & 5.2 \\
\hline DKY AGENT & 0.0 & 0.0 & 0.0 & .3 & .4 & .4 & .4 & .4 & .5 \\
\hline ELEC- FWR & 0.0 & 0.0 & 0.0 & 0.0 & 0.0 & 0.0 & 0.0 & 0.0 & 0.0 \\
\hline TOTAR REUS & 0.0 & 0.0 & 0.0 & 14.1 & 15.2 & 16.4 & 17.7 & 19.2 & 20.7 \\
\hline VAFITAELE COST & & & & & & & & & \\
\hline FEEISTOCK & 0.0 & 0.0 & 0.0 & 5.5 & 6.0 & 6.4 & 6.9 & 7.5 & 8.1 \\
\hline woobi & 0.0 & 0.0 & 0.0 & .5 & .5 & .6 & .6 & .7 & .7 \\
\hline DEY AGENT & 0.0 & 0.0 & 0.0 & .3 & .4 & .4 & .4 & .4 & .5 \\
\hline ELECI & 9.0 & 0.0 & 0.0 & .4 & .4 & .4 & .5 & .5 & .5 \\
\hline DTHES & 0.0 & 0.0 & 0.0 & .6 & .7 & .7 & .8 & .9 & .9 \\
\hline TOTA: VC & 2.0 & 0.0 & 0.0 & 7.3 & 7.9 & 8.6 & 9.2 & 10.0 & 10.8 \\
\hline$\dot{F}$ IXED casts & & & & & & & & & \\
\hline LAHOS̃ & 0.0 & 0.0 & 0.0 & .9 & 1.0 & 1.1 & 1.2 & 1.3 & 1.4 \\
\hline DEPREC & 0.0 & 0.0 & 0.0 & 2.5 & 3.6 & 3.4 & 3.4 & 3.4 & .1 \\
\hline G $8 A$ & 0.0 & 0.0 & 0.0 & .4 & .4 & .4 & .5 & .5 & .6 \\
\hline ОТНER & 0.0 & 0.0 & 0.0 & .3 & .3 & .3 & .3 & .3 & .4 \\
\hline TOTAL FC & 0.0 & 0.0 & 0.0 & 4.1 & 5.3 & 5.3 & 5.4 & 5.5 & 2.4 \\
\hline STAKTUF COSTS & 0.0 & 0.0 & .7 & 0.0 & 0.0 & 0.0 & 0.0 & 0.0 & 0.0 \\
\hline TOTAL CPER COSTS & 0.0 & 0.0 & .7 & 11.4 & 13.2 & 13.8 & 14.6 & 15.5 & 13.2 \\
\hline OFERATING INCOME & 0.0 & 0.0 & -.7 & 2.6 & 2.0 & 2.6 & 3.1 & 3.6 & 7.5 \\
\hline INTEREST & 0.0 & 0.0 & 0.0 & 2.1 & 2,1 & 2.0 & 1.8 & 1.7 & 1.5 \\
\hline INC, BEF, LCF/TAX & 0.0 & 0.0 & -.7 & .5 & -.1 & .6 & 1.3 & 1.9 & 6.0 \\
\hline LCF USEH & 0.0 & 0.0 & 0.0 & .5 & 0.0 & .3 & 0.0 & 0.0 & 0.0 \\
\hline TAXAELE INCOME & 0.0 & 0.0 & -.7 & 0.0 & -.1 & .4 & 1.3 & 1.9 & 6.0 \\
\hline ITC USED & 0.0 & 0.0 & 0.0 & 0.0 & 0.0 & .2 & .6 & 1.0 & 1.4 \\
\hline TAXES FAID & 0.0 & 0.0 & 0.0 & 0.0 & 0.0 & 0.0 & 0.0 & 0.0 & 1.6 \\
\hline INCOME AFTER: TAX & 0.0 & 0.0 & -.7 & .5 & $\because .1$ & .6 & 1.3 & 1.9 & 4.3 \\
\hline DEPREC & 0.0 & 0.0 & $\cdot 0.0$ & 2.5 & 3.6 & 3.4 & 3.4 & 3.4 & .1 \\
\hline CHG IN OTH ASSETS & 0.0 & 0.0 & -1.2 & 0.0 & 0.0 & 0.0 & 0.0 & 0.0 & 0.0 \\
\hline FRIHL REFAY & 0.0 & 0.0 & 0.0 & $\cdots .6$ & -.7 & -.8 & -.9 & -1.1 & -1.2 \\
\hline CHG IN WC & 0.0 & 0.0 & -2.0 & -.1 & $\cdots, 1$ & $\cdots, 1$ & -.1 & -.1 & -.1 \\
\hline CHS IN WANK LINE & 0.0 & 0.0 & 1.0 & .0 & .1 & .1 & .1 & .1 & .1 \\
\hline ENG cos $\mathrm{T}$ & -.6 & 0.0 & 0.0 & 0.0 & 0.0 & 0,0 & 0.0 & 0.0 & 0.0 \\
\hline $\operatorname{consTR} \cos \mathrm{T}$ & 0.0 & --9.4 & -4.6 & 0.0 & 0.0 & 0.0 & 0.0 & 0.0 & 0.0 \\
\hline COHGTK INT & 0.0 &.- .7 & -1.9 & 0.0 & 0.0 & 0.0 & 0.0 & 0.0 & 0.0 \\
\hline DELT FIN & 0.0 & $\% .6$ & 4.8 & 0.0 & 0.0 & 0.0 & 0.0 & 0.0 & 0.0 \\
\hline GNINUAL CASH FLOU! & $\cdots, 0$ & -2.5 & -4.5 & 2.4 & 2.9 & 3.2 & 3.7 & 4.2 & 3.1 \\
\hline CUMUL LASH FLUW &.$- c$ & -3.1 & -7.6 & -5.2 & $-2,4$ & .8 & 4.5 & 8.8 & 11.9 \\
\hline
\end{tabular}




\section{Exhibit 4.3 (continued)}

INCOME AND CASH FLOW (\$ HIL)

\begin{tabular}{|c|c|c|c|c|c|c|c|c|c|}
\hline REVENUES & $1: 91$ & 1992 & 1993 & 1994 & 1995 & 1996 & 1997 & 1990 & 1999 \\
\hline ETHANOL & 16.2 & 17.5 & 18.9 & 20.4 & 22.0 & 23.0 & 25.7 & 27.7 & 29.9 \\
\hline DISG & 5.7 & 6.1 & 6.6 & 7.1 & 7.7 & 8.3 & 9.0 & 9.7 & 10.5 \\
\hline DWYY ALENT & .5 & .6 & .6 & .7 & .7 & .8 & .8 & .9 & 1.0 \\
\hline ELECT FWF & 0.0 & 0.0 & 0.0 & 0.0 & 0.0 & 0.0 & 0.0 & 0.0 & 0.0 \\
\hline TOTAL REUS & 22.3 & 24.1 & 26.1 & 28.1 & 30.4 & 32.8 & 35.5 & 38.3 & 41.4 \\
\hline \multicolumn{10}{|l|}{ UAFIABLE COST } \\
\hline FEEIISTOCK & 8.7 & 9.4 & 10.2 & 11.0 & 11.9 & 12.7 & 13.7 & 15.0 & 16.2 \\
\hline WOON & .8 & .9 & .9 & 1.0 & 1.1 & 1.2 & 1.3 & 1.4 & 1.5 \\
\hline DFYY AGENT & .5 & .6 & .6 & .7 & .7 & .8 & .0 & .9 & 1.0 \\
\hline ELECT & .6 & .6 & .7 & .7 & .8 & .8 & .9 & 1.0 & 1.1 \\
\hline OTHEF & 1.0 & 1.1 & 1.2 & 1.3 & 1.4 & 1.5 & 1.6 & 1.7 & 1.9 \\
\hline TOTAL UC: & 11.6 & 12.6 & 13.6 & 14.7 & 15.8 & 17.1 & 18.5 & 20.0 & 21.5 \\
\hline \multicolumn{10}{|l|}{ FIXEL $\quad \cos T S$} \\
\hline LABOI: & 1.5 & 1.6 & 1.7 & 1.9 & 2.0 & 2.2 & 2.4 & 2.6 & 2.8 \\
\hline DEFREC & .1 & .1 & .1 & .1 & .1 & .1 & .1 & .1 & .1 \\
\hline$G \& A$ & .6 & .6 & .7 & .0 & .0 & .9 & 1.0 & 1.0 & 1.1 \\
\hline OTHER: & .4 & .4 & .5 & .5 & .5 & .6 & .6 & .7 & .7 \\
\hline TOTAL FC & 2.6 & 2.8 & 3.0 & 3.2 & 3.5 & 3.7 & 4.0 & 4.4 & 4.7 \\
\hline STARTUP COSTS & 0.0 & 0.0 & 0.0 & 0.0 & 0.0 & 0.0 & 0.0 & 0.0 & 0.0 \\
\hline TOTAL OFEK COSTS & 14.2 & 15.3 & 16.6 & 17.9 & 19.3 & 20.8 & 22.5 & 24.3 & 26.2 \\
\hline OPEFATING INCOME & 8.1 & 8.8 & 9.5 & 10.3 & 11.1 & 12.0 & 12.9 & 14.0 & 15.1 \\
\hline INTEREST & 1.4 & 1.1 & .9 & .6 & .3 & .3 & .3 & .3 & .3 \\
\hline INC. BEF, LCF/TAX & 6.8 & 7.6 & 8.6 & 9.7 & 10.8 & 11.7 & 12.6 & 13.7 & 14.8 \\
\hline LCF USED & 0.0 & 0.0 & 0.0 & 0.0 & 0.0 & 0.0 & 0.0 & 0.0 & 0.0 \\
\hline TAXABLE INCOME & 8.8 & 7.6 & 8.6 & 9.7 & 10.8 & 11.7 & 12.6 & 13.7 & 14.8 \\
\hline $\begin{array}{l}\text { ITC USEI } \\
\text { TAXES PAID }\end{array}$ & $\begin{array}{l}0.0 \\
3.4\end{array}$ & 0.0 & 0.0 & 0.0 & 0.0 & 0.0 & 0.0 & 0.0 & $\begin{array}{l}0.0 \\
7.4\end{array}$ \\
\hline & 3.4 & 38 & & 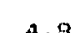 & $=1$ & $=0$ & 87 & 60 & \\
\hline & 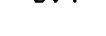 & 3.0 & 4.0 & 7.0 & . & 3.8 & 0.3 & 0.0 & 1.4 \\
\hline TEFFEC & $\cdot 1$ & .1 & .1 & .1 & .1 & .1 & .1 & .1 & .1 \\
\hline CHG IN OTH ASSETS & .0 .0 & 0.0 & 0.0 & 0.0 & 0.0 & 0.0 & 0.0 & 0.0 & 0.0 \\
\hline FRINE REFAY & -1.4 & -1.6 & -1.9 & -2.2 & 0.0 & 0.0 & 0.0 & 0.0 & 0.0 \\
\hline CHG IN WC & $\cdots .2$ & -.2 & -.2 & -.2 & -.2 & -.2 & -.2 & -.3 & -.3 \\
\hline CHG IN LIANK LINE & .1 & .1 & .1 & .1 & .1 & .1 & .1 & .1 & .1 \\
\hline ENG $\cos \mathrm{T}$ & 0.0 & 0.0 & 0.0 & 0.0 & 0.0 & 0.0 & $0.0^{\circ}-1-1-1$ & 0.0 & 0.0 \\
\hline consti: $\cos T$ & 0.0 & 0.0 & 0.0 & 0.0 & 0.0 & 0.0 & 0.0 & 0.0 & 0.0 \\
\hline COHSTR INT & 0.0 & 0.0 & 0.0 & 0.0 & 0.0 & 0.0 & 0.0 & 0.0 & 0.0 \\
\hline DEETT FIN & 0.0 & 0.0 & 0.0 & 0.0 & 0.0 & 0.0 & 0.0 & 0.0 & 0.0 \\
\hline $\begin{array}{l}\text { ANFIUAL CASH FLOW } \\
\text { CIMUUL. CAEH FLOW }\end{array}$ & $\begin{array}{r}2.0 \\
13.9\end{array}$ & $\begin{array}{r}2.2 \\
16.0\end{array}$ & $\begin{array}{r}2.4 \\
18.4\end{array}$ & $\begin{array}{r}2.6 \\
21.0\end{array}$ & $\begin{array}{r}5.4 \\
26.4\end{array}$ & $\begin{array}{r}5.0 \\
32.2\end{array}$ & $\begin{array}{r}6.3 \\
38.3\end{array}$ & $\begin{array}{r}6.8 \\
4=.3\end{array}$ & 7.3 \\
\hline
\end{tabular}




\section{Exhibit 4.4}

WALANCE SHELT (T HIL)

\begin{tabular}{|c|c|c|c|c|c|c|c|c|c|}
\hline & $1 ! 92$ & 1783 & 1984 & 1985 & 1936 & $198 \%$ & 1988 & 1989 & 1990 \\
\hline $\begin{array}{l}\text { CU:FRENT ASGETS } \\
\text { CASH }\end{array}$ & 0.0 & 0.0 & 0.0 & 2.4 & 5.2 & 8.4 & 12.2 & 16.4 & 19.5 \\
\hline ACCTS REC & 0.0 & 0.0 & 1.1 & 1.2 & 1.3 & 1.4 & 1.5 & $\begin{array}{l}10.4 \\
1.6\end{array}$ & 1.7 \\
\hline FESTE INU & 0.0 & 0.0 & .2 & .2 & .2 & .2 & .2 & .2 & .2 \\
\hline DFY AGT INU & 0.0 & 0.0 & .0 & .0 & .0 & .0 & .0 & .0 & .0 \\
\hline WOOH INV & 0.0 & 0.0 & .0 & .0 & .0 & .0 & .0 & .0 & .0 \\
\hline ETH INY & 0.0 & 0.0 & .4 & .4 & .4 & .4 & .4 & .4 & .4 \\
\hline GIS INU & 0.0 & 0.0 & .1 & .1 & .1 & .1 & .1 & .1 . & .1 \\
\hline DTHEF: & 0.0 & 0.0 & .2 & .3 & .3 & .3 & .3 & .3 & .4 \\
\hline TOTAL CA & 0.0 & 0.0 & 2.1 & 4.6 & 7.5 & 10.9 & 14.7 & 19.1 & 22.4 \\
\hline FIKED ASSETS & & & & & & & & & \\
\hline $\begin{array}{l}\text { EQUIF } \\
\text { BUILUING }\end{array}$ & 0.6 & $\begin{array}{r}9.9 \\
.9\end{array}$ & $\begin{array}{r}15.7 \\
1.4\end{array}$ & $\begin{array}{r}13.4 \\
1.2\end{array}$ & $\begin{array}{l}9.9 \\
1.1\end{array}$ & $\begin{array}{l}6.6 \\
1.0\end{array}$ & $\begin{array}{r}3.3 \\
.9\end{array}$ & $\begin{array}{r}0.0 \\
.8\end{array}$ & $\begin{array}{r}0.0 \\
.7\end{array}$ \\
\hline TOTAL FA & .6 & 10.7 & 17.1 & 14.6 & 11.0 & 7.6 & 4.2 & .8 & .7 \\
\hline OTHER ASSETS & 0.0 & 0.0 & 1.2 & 1.2 & 1.2 & 1.2 & 1.2 & 1.2 & 1.2 \\
\hline TOTAL ASSETS & .6 & 10.7 & 20.4 & 20.4 & 19.7 & 19.7 & 20.1 & 21.0 & 24.3 \\
\hline $\begin{array}{c}\text { CURRENT LIAB } \\
\text { FUSTK FAY }\end{array}$ & 0.0 & 0.0 & 0.0 & 0.0 & 0.0 & 0.0 & 0.0 & & 0.0 \\
\hline DRY AGT. PAY & 0.0 & 0.0 & .0 & .0 & .0 & .0 & .0 & .0 & .0 \\
\hline WOOD PAY & 0.0 & 0.0 & 0.0 & 0.0 & 0.0 & 0.0 & 0.0 & 0.0 & 0.0 \\
\hline OTHER CL & 0.0 & 0.0 & .1 & .1 & $:^{1}$ & .1 & .2 & .2 & .2 \\
\hline TOTAL CL & 0.0 & 0.0 & .1 & .2 & .2 & .2 & .2 & .2 & .2 \\
\hline BANK LINE & 0.0 & 0.0 & 1.0 & 1.0 & 1.1 & 1.1 & 1.2 & 1.3 & 1.3 \\
\hline LT DEET & $c .0$ & 7.6 & 12.4 & 11.8 & 11.1 & 10.4 & 9.5 & 8.4 & 7.2 \\
\hline EQUITY & .6 & 3.1 & 6.9 & 7.4 & 7.3 & 8.0 & 9.3 & 11.2 & 15.5 \\
\hline TO-AL LIAHILITY/EQ & .6 & 10.7 & 20.4 & 20.4 & 19.7 & 19.7 & 20.1 & 21.0 & 24.3 \\
\hline
\end{tabular}


Exhibit 4.4 (continued)

\begin{tabular}{|c|c|c|c|c|c|c|c|c|c|}
\hline \multicolumn{10}{|l|}{ CURAENT ASEETS } \\
\hline CASH & 24.5 & 23.6 & 26.0 & 20.6 & 34.0 & 39.8 & 46.1 & 52.9 & 60.2 \\
\hline ACCTS REC & 1.8 & 2.0 & 2.2 & 2.4 & 2.6 & 2.0 & 3.0 & 3.2 & 3.5 \\
\hline FISTK INU & .2 & .2 & .2 & .2 & .2 & .2 & .2 & .2 & .2 \\
\hline DRYY AGT INV & .0 & .0 & .0 & .0 & .0 & .0 & .0 & .0 & .0 \\
\hline WoOn INU & .0 & .0 & $\therefore$ & .0 & .0 & .0 & .0 & .0 & .0 \\
\hline ETH INU & .4 & .4 & .4 & .4 & .4 & .4 & .4 & .4 & .4 \\
\hline IIIG INU & .1 & .1 & .1 & .1 & .1 & +1 & +1 & .1 & $\cdot 1$ \\
\hline OTHEK & .4 & .4 & .5 & .5 & .5 & .6 & .6 & .7 & .7 \\
\hline TOTAL CA & 27.5 & 26.9 & 29.5 & 32.3 & 37.8 & 43.9 & 50.5 & 57.6 & 65.2 \\
\hline FJXED ASSETS & 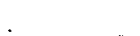 & & & & & & & & \\
\hline EQUIF & 3.0 & 0.0 & 0.0 & 0.0 & 0.0 & 0.0 & 0.0 & 0.0 & 0.0 \\
\hline EUILDING & .6 & .5 & .4 & .4 & .3 & .2 & .1 & .1 & 0.0 \\
\hline TOTAL FA & .6 & .5 & .4 & .4 & .3 & .2 & .1 & .1 & 0.0 \\
\hline OUHER ASSETS & 1.2 & 1.2 & 1.2 & 1.2 & 1.2 & 1.2 & 1.2 & 1,2 & 1.2 \\
\hline TCTAL ASSETS & 25.3 & 28.6 & 31.1 & 33.8 & 39.4 & 45.4 & 51.8 & 58.8 & 66.4 \\
\hline CLTRRENT LIAB & & & & & & & & & \\
\hline FISTK FAAY & .0 .0 & 0.0 & 0.0 & 0.0 & 0.0 & 0.0 & 0.0 & 0.0 & 0.0 \\
\hline DFY AGT F'AY & .0 & .0 & $\therefore 1$ & .1 & .1 & 1 & .1 & .1 & 1 \\
\hline WOOD FAY & 0.0 & 0.0 & 0.0 & 0.0 & 0.0 & 0.0 & 0.0 & 0.0 & 0.0 \\
\hline OTHER CL & .2 & .2 & .2 & .3 & .3 & .3 & .3 & .3 & .4 \\
\hline TOTAL CL & .2 & .3 & .3 & .3 & .3 & .4 & .4 & .4 & .4 \\
\hline BANK LINE & 8.4 & 1.5 & 1.6 & 1.7 & 1.8 & 1.7 & 2.0 & 2.1 & 2.3 \\
\hline LT NEET & 5.8 & 4.1 & 2.2 & 0.0 & 0.0 & 0.0 & 0.0 & 0.0 & 0.0 \\
\hline EGUITY & $1 \mathrm{ij} .9$ & 22.7 & 27.0 & 31.9 & 37.3 & 43.1 & 49.4 & .56 .3 & 63.7 \\
\hline TETAL LIALILITY/EQ & $2 \dot{3} \cdot 3$ & 28.6 & 31.1 & 33.8 & 39.4 & 45.4 & 51.8 & 58.8 & 66.4 \\
\hline
\end{tabular}




\section{Exhibit 4.5}

BOURCES ANL USES OF FUNDS (\$ MIL)

\begin{tabular}{|c|c|c|c|c|c|c|c|c|c|}
\hline SCUFCES & 1582 & 1903 & 1984 & 1935 & 1986 & 1987 & 1988 & 1909 & 1990 \\
\hline REVE VUES & 0.0 & 0.0 & 0.0 & 14.1 & 15.2 & 16.4 & 17.7 & 19.2 & 20.7 \\
\hline IIEET FINANCING & 0.0 & 7.6 & 4.8 & 0.0 & 0.0 & 0.0 & 0.0 & 0.0 & 0.0 \\
\hline IEECK IN WC & 0.0 & 0.0 & 0.0 & 0.0 & 0.0 & 0.0 & 0.0 & 0.0 & 0.0 \\
\hline INCE IN BK LINE & 0.0 & 0.0 & 1.0 & .0 & .1 & .1 & .1 & .1 & .1 \\
\hline ERUITY INUEST & .0 & 2.5 & 4.5 & 0.0 & 0.0 & 0.0 & 0.0 & 0.0 & 0.0 \\
\hline TOTAL & .6 & 10.1 & 10.3 & 14.1 & 15.3 & 16.5 & 17.8 & 19.2 & 20.8 \\
\hline $\begin{array}{l}\text { USES } \\
\text { OFEF COSTS (- NEFF) }\end{array}$ & 0.0 & 0.0 & 0.0 & 8.9 & 9.6 & 10,4 & 11.2 & 12.1 & 13,1 \\
\hline INTEREST & 0.0 & 0.0 & 0.0 & 2.1 & 2.1 & 2.0 & 1.8 & 1.7 & 1.5 \\
\hline TAXES PAID & 0.0 & 0.0 & 0.0 & 0.0 & 0.0 & 0.0 & 0.0 & 0.0 & 1.6 \\
\hline FEINC, REPAY & 0.0 & 0.0 & 0.0 & .6 & .7 & .8 & .9 & 1.1 & 1.2 \\
\hline INCF:. IN WC & 0.0 & 0.0 & 2.0 & .1 & .1 & .1 & .1 & .1 & .1 \\
\hline IEECP. IN EKK LINE & 0.0 & 0.0 & 0.0 & 0.0 & 0.0 & 0.0 & 0.0 & 0.0 & 0.0 \\
\hline INCF:. IN OTH ASSETS & 0.0 & 0.0 & 1.2 & 0.0 & 0.0 & 0.0 & 0.0 & 0.0 & 0.0 \\
\hline ENG:STARTUP & .6 & 0.0 & .7 & 0.0 & 0.0 & 0.0 & 0.0 & 0.0 & 0.0 \\
\hline CONSTR, COSTS & 0.0 & 9.4 & 4.6 & 0.0 & 0.0 & 0.0 & 0.0 & 0.0 & 0.0 \\
\hline CONSTR. INT. & 0.0 & .7 & 1.8 & 0.0 & 0.0 & 0.0 & 0.0 & 0.0 & 0.0 \\
\hline EQUITY FETUFN \& CASH & 0.0 & 0.0 & 0.0 & 2.4 & 2.8 & 3.2 & 3.7 & 4.2 & 3.1 \\
\hline TUTAL & .6 & 10.1 & 10.3 & 14.1 & 15.3 & 16.5 & 17.8 & 19.2 & 20.8 \\
\hline
\end{tabular}


Exhibit 4.5 (continued)

SOUFCES AND USES OF FUNISS ( $\$$ MIL)

\begin{tabular}{|c|c|c|c|c|c|c|c|c|c|}
\hline & & & & & & & & & \\
\hline S0'JRCES & 1991 & 1992 & 1993 & 1994 & 1995 & 1996 & 1997 & 1998 & 1999 \\
\hline REVEMUES & 22.3 & 24.1 & 26.1 & 28.1 & 30.4 & 32.8 & 35.5 & 38.3 & 41.4 \\
\hline IIEBT FINANCING & 0.0 & 0.0 & 0.0 & 0.0 & 0.0 & 0.0 & 0.0 & 0.0 & 0.0 \\
\hline IEECE IN WC & 0.0 & 0.0 & 0.0 & 0.0 & 0.0 & 0.0 & 0.0 & 0.0 & 0.0 \\
\hline INCR IN GK LINE & .1 & .1 & .1 & .1 & .1 & .1 & .1 & .1 & .1 \\
\hline EQUITY INUEST & 0.0 & 0.0 & 0.0 & 0.0 & 0.0 & 0.0 & 0.0 & 0.0 & 0.0 \\
\hline TOTAL & 22.4 & 24.2 & 26.2 & 28.2 & 30.5 & 32.9 & 35.6 & 38.4 & 41.5 \\
\hline $\begin{array}{l}\text { USES } \\
\text { DPER COSTS (- IEFF) }\end{array}$ & 14,1 & 15.3 & 16.5 & 17.8 & 19.2 & 20.8 & 22,4 & 24,2 & 26.2 \\
\hline INTEREST & 2.4 & 1.1 & .9 & .6 & .3 & .3 & .3 & .3 & .3 \\
\hline TAXES PAID & 3.4 & 3.8 & 4.3 & 4.8 & 5.4 & 5.9 & 6.3 & 6.8 & 7.4 \\
\hline FRINC. REFAY & 1.4 & 1.6 & 1.9 & 2.2 & 0.0 & 0.0 & 0.0 & 0.0 & 0.0 \\
\hline INCR. IN WC & .2 & .2 & .2 & .2 & .2 & .2 & .2 & .3 & .3 \\
\hline DECR. IN BK LINE & 0.0 & 0.0 & 0.0 & 0.0 & 0.0 & 0.0 & 0.0 & 0.0 & 0.0 \\
\hline INCF. IN OTH ASSETS & 0.0 & 0.0 & 0.0 & 0.0 & 0.0 & 0.0 & 0.0 & 0.0 & 0.0 \\
\hline ENG/STAETUF & 0.0 & 0.0 & 0.0 & 0.0 & 0.0 & 0.0 & 0.0 & 0.0 & 0.0 \\
\hline CONSTF. COSTS & 0.0 & 0.0 & 0.0 & 0.0 & 0.0 & 0.0 & 0.0 & 0.0 & 0.0 \\
\hline CONSTR. INT. & 0.0 & 0.0 & 0.0 & 0.0 & 0.0 & 0.0 & 0.0 & 0.0 & 0.0 \\
\hline EQUITY FETUFN \& CASH & 2.0 & 2.2 & 2.4 & 2.6 & 5.4 & 5.8 & 6.3 & 6.8 & 7.3 \\
\hline TOTAL & 22.4 & 24.2 & 26.2 & 28.2 & 30.5 & 32.9 & 35.6 & 38.4 & 41.5 \\
\hline
\end{tabular}


Exhibit 4.6

Sensitivity of Corn-to-Ethanol Project Performance

to Changes in Corn and Ethanol Price

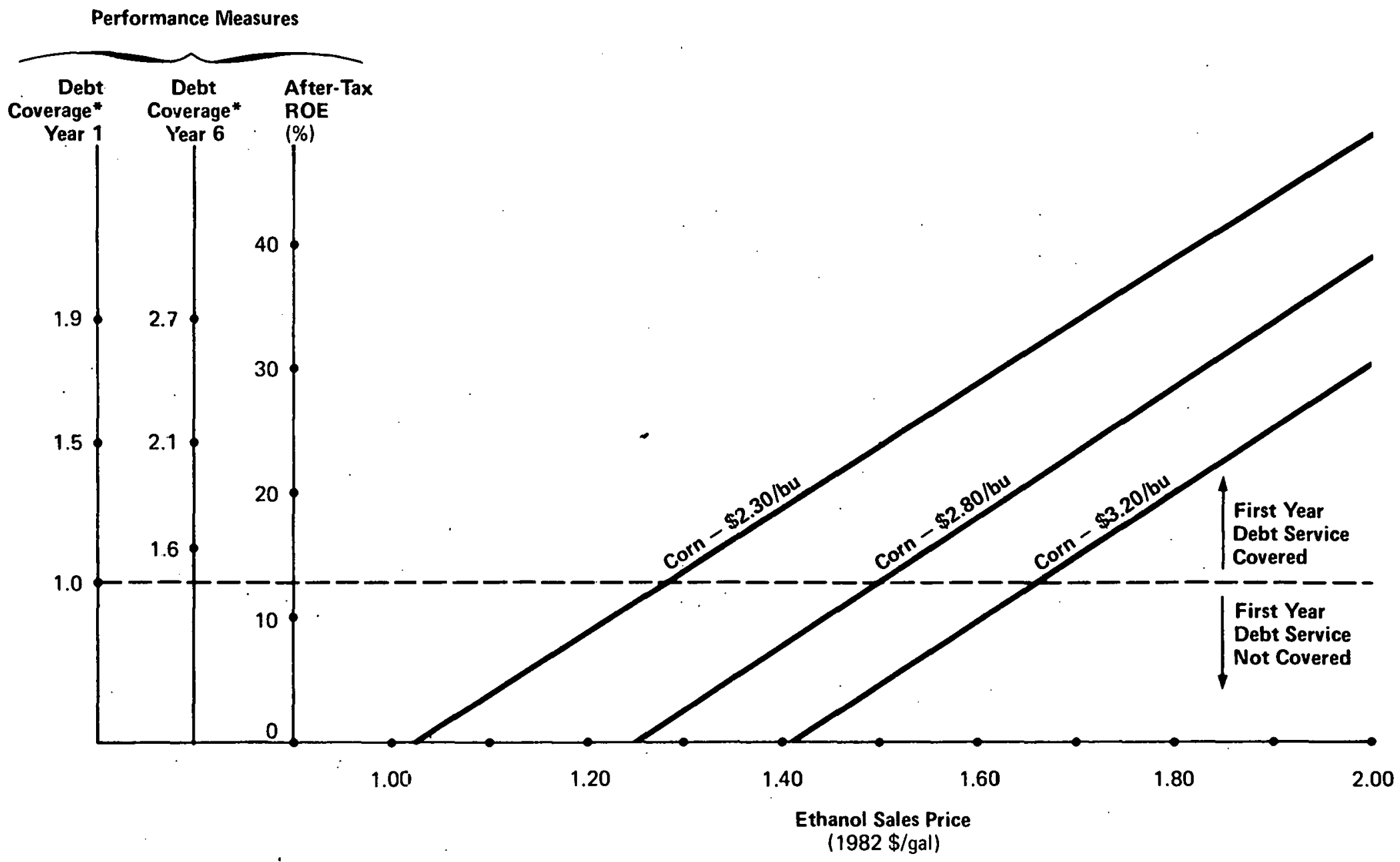

- Debt coverage $=$ operating cash flow $\div$ debt sersice. 
Exhibit 4.7

Downside Risk Analyses

5-Million GPY Corn-to-Ethanol Project

(First Operating Year Cash Flow Adequacy)

First Year Cash Flow

(\$ mil)

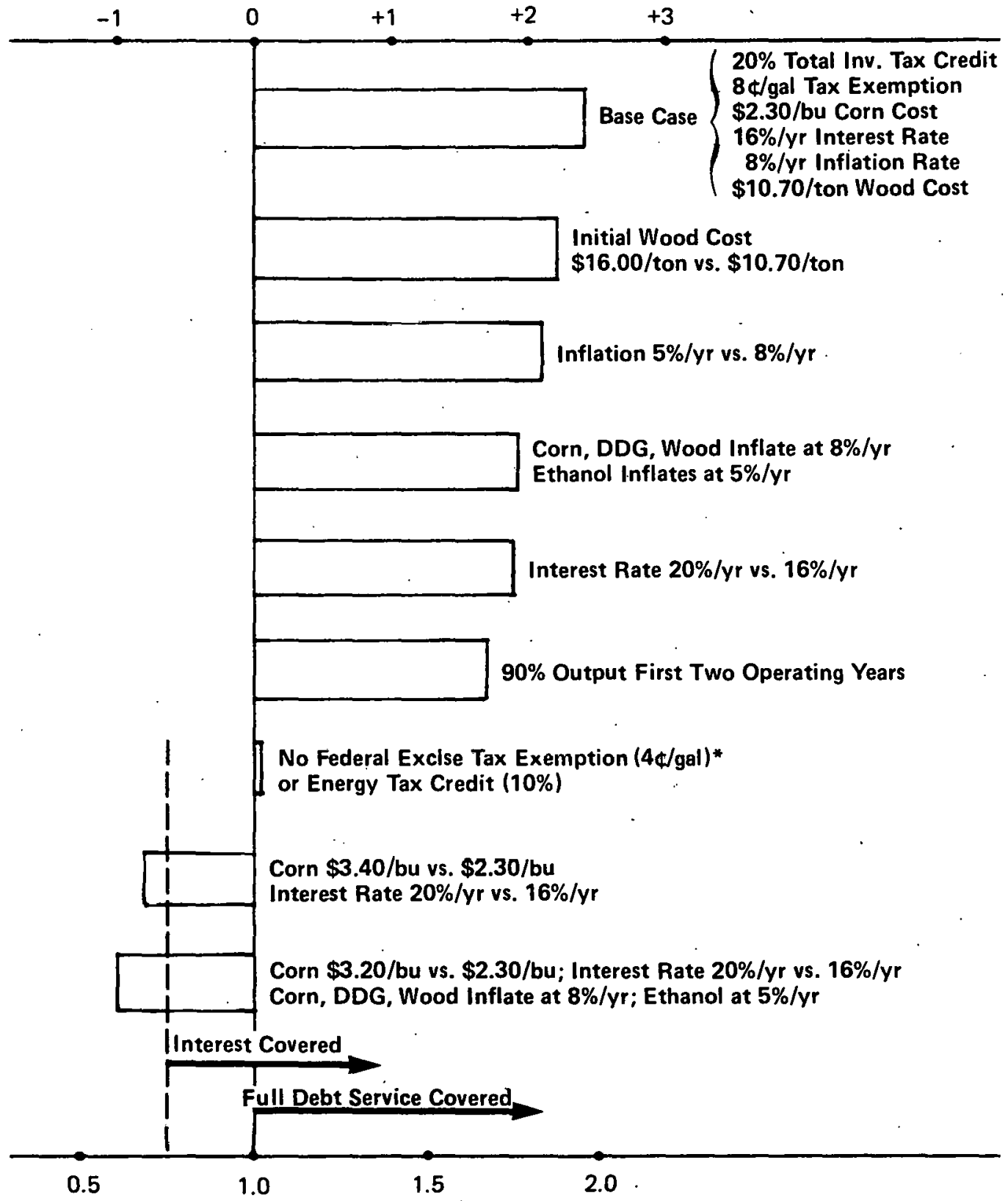

First Year Debt Coverage Ratio* *

*Results similar to retention of federal exemption, but with loss of the statc exemption.

" "Debt coverage ratio $=$ operating cash flow $\div$ debt service requlrement. 
Exhibit 4.8a

10-Year Average Cash Prices for Selected Grains and Unleaded Gasoline

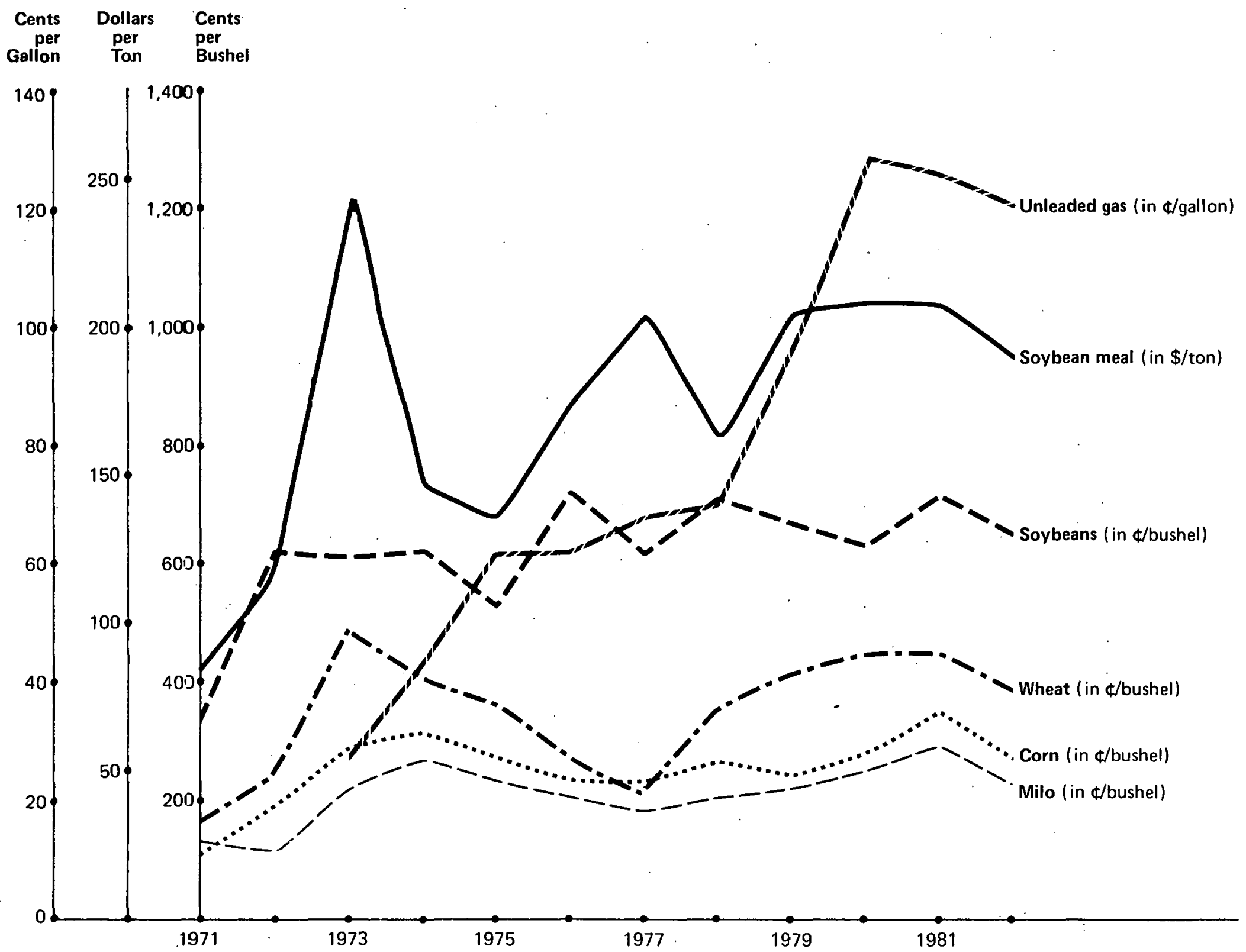




\section{Exhibit 4.8b}

\section{Long-Term Historical Cash Price Trends for Selected Grains}

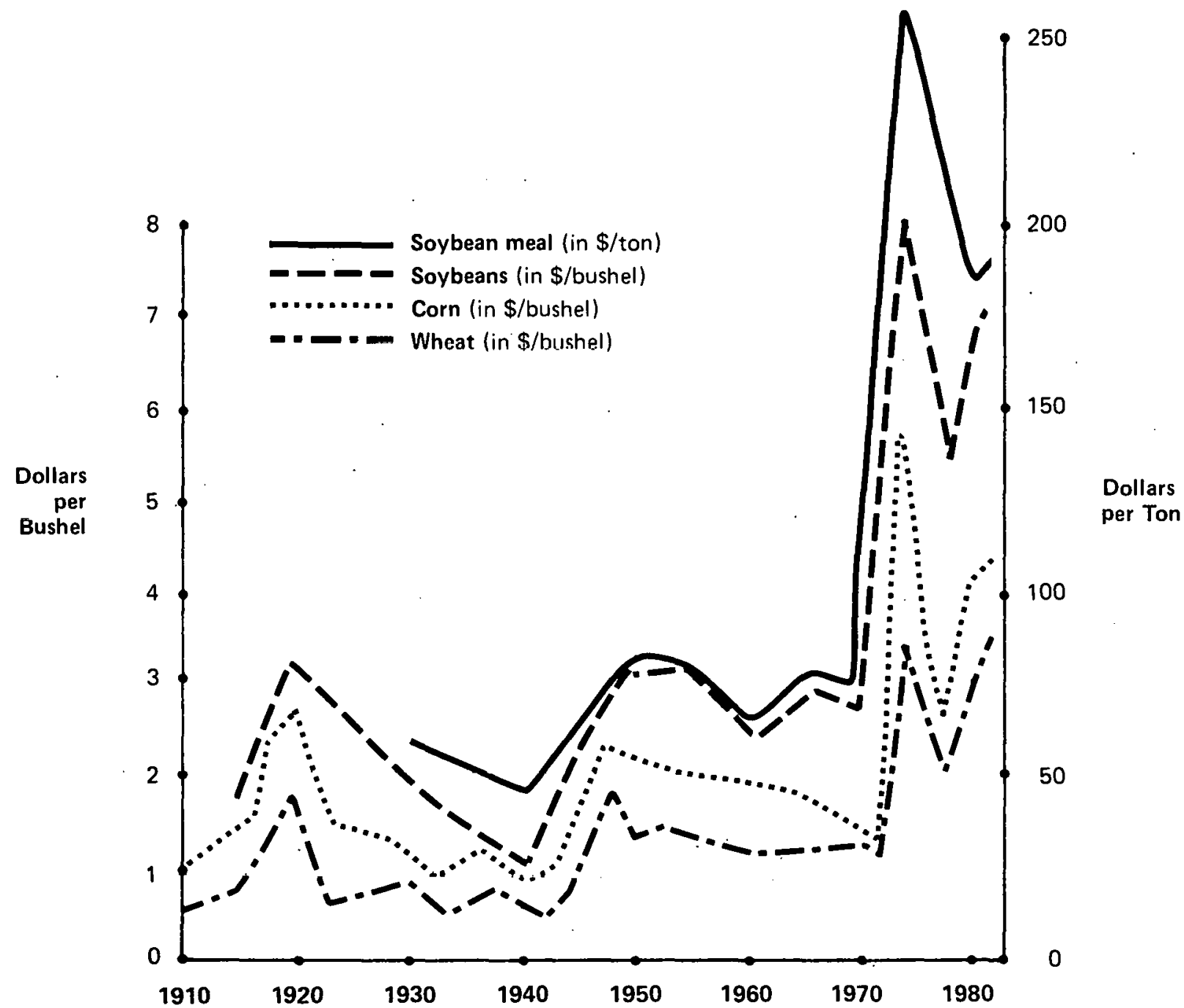


Sun Belt Energy Corporation views the analysis of the effects of the proposed plant on the environment, health, safety, and socioeconomic conditions of Sardis, Mississippi, and Panola County as an important element of the feasibility study. As residents of Panola county, the project's principals are concerned with the plant's impact on their community.

The firm has been in close communication with government officials and prominent community leaders. The plant has received wide publicity, including newspaper and television coverage. The local community overwhelmingly supports the construction of the proposed plant, primarily because of its positive economic effect on the community. Moreover, since the plant site lies within a designated industrial zone, no public opposition to its location is expected.

Since there are few unknowns regarding the plant's process and the site, we believe our assessments of impacts and project costs to be reliable. The plant's process has been used on a commercial scale in many fuel ethanol plants across the country. The effluents and emissions from the plant are well known. A wood-burning boiler was selected because of the availablitty of vast alluurits of surplus waste wood and scrub timber in the vicinity of the plant, and because its air emissions would be environmentally benign. Furthermore, the site is in a countydesignated industrial area with excellent transportation access and available utilities.

This section covers the environmental, health, safety, and social and economic considerations regarding the proposed plant. It also includes a description of regulatory and permit requirements and a management schedule for meeting them. 


\section{1 SITE DESCRIPTION}

The proposed plant site has excellent industrial potential. It is located in North Panola County, Mississippi, about 45 miles south of Memphis, Tennessee, and approximately 168 miles north of Jackson, Mississippi. Panola County, organized in 1836, ranks 20 th in land area in Mississippi and covers 704 square miles. The Tallahatchie River forms a dividing line between North and South Panola County. The county seat for North Panola County is Sardis. Relevant population and economic data for Panola county and Sardis, Mississippi, are shown in Exhibit 5.1.

The plant site is located in sardis and is part of the Panola County industrial park of 120 acres. The site is served by a county road, and is about 2 miles from access to Interstate 55 to the east and 1 mile from access to U.S. Highway 51 to the west. The mainline track of the Illinois Central Railroad fronts the site on the west.

The industrial park is an abandoned prisoner-of-war camp that was used during World War II. The camp was decommissioned during the war, and all structures were removed. The site extends across relatively level pasture land with a stream bed that is dry most of the year.

There are no archaeological sites on or near the plant site. Extensive work has been done on archaeology in the state of Mississippi by the University of Mississippi, the Mississippi Archaeological Association, Harvard University, the National Park Service, and other renowned archaeological groups. Although Mississippi is rich with artifacts of ancient Indian cultures, the site of archaeological interest nearest to the plant site is in Batesvillc, Miooiooippi, about 10 milco oouth of Eardio:

\subsection{ENVIRONMENTAL CONSIDERATIONS * *}

All environmental discharges from the plant will be. within the limitations allowed by the Mississippi Air

* Calvin S. Brown. Archaeology of Mississippi. AMS Press, New York, 1973.

** Prepared by Bechtel Petroleum, Inc. 
and Water Pollution Control Commission. Appropriate commercially available pollution abatement equipment and controls will be employed to meet these requirements. The anticipated environmental discharges are discussed below.

\section{Air Emissions}

Dust generated from the normal operation of the hammer mill, conveyors, elevators, DDGS dryer, and storage areas will be aspirated. The dust-laden streams will be passed through centrifugal cyclones to minimize fire hazard and particulate emissions.

The wood-fired boiler system will include a separate cyclone to remove particulate matter from the spent flue gases discharged through the DDG dryer system and into the atmosphere. The cyclone will have a removal efficiency of 94-95 percent. In the unlikely event that the cyclone alone proves to be inadequate, the boiler system can be retrofitted with a bag filter in series with the cyclone. Emission of $\mathrm{NO}_{x}$ will be minimal because the fluidized-bed combustion temperature will be limited to $1500-1600^{\circ} \mathrm{F}$. Since wood fuel is normally low in sulfur content (relative to coal), no $\mathrm{SO}_{2}$ removal equipment will be required. The $\mathrm{SO}_{2}$ and $\mathrm{CO}$ emissions will be within allowable limits.

Some hydrocarbon emissions will come from the product surge tanks and the denaturant tanks. The main alcohol storage tanks will have internal floating roofs to minimize vapor losses from tank breathing or displaced vapors during filling operations. The gasoline storage tank will be provided with a vapor recovery balance-back system to eliminate the escape of gasoline vapors into the atmosphere during transfer operations from tank truck to storage. The minimal amount of vapor displaced from the alcohol tank trucks during filling operations will be piped away from the truck rack area to minimize fire hazard. Since vapor losses from these tanks is insignificant relative to state environmental standards, a vapor recovery system will not be necessary.

\section{Liquid Effluents and Wastes}

Approximately $70 \mathrm{gpm}$ of process and sanitary wastes will be piped to an activated sludge wastewater treatment 
system. The wastewater will flow initially to an equalization basin for stabilization, and then to two aeration basins connected in series. Compressed air supplied through a diffuser system provides the oxygen necessary to satisfy the demands of the micro-organisms responsible for consuming the organic waste material. The basins will be constructed of concrete to prevent the possibility of wastewater leaching into the groundwater.

The BOD level of the treated effluent will be reduced from $1000 \mathrm{mg} / 1$ in the combined wastewater stream $(2,000 \mathrm{mg} / 1$ in stripper bottom and evaporator condensate) to $30 \mathrm{mg} / \mathrm{l}$ for eventual discharge into McIvor Canal, which flows approximately 2 miles south of the plant site. Computer model studies (to be made by the Mississippi Air and Water Pollution Control Commission) will evaluate the possibility of discharging the treated effluent into the dry stream bed that runs from the plant site to the canal. Allowances have been made to provide a 6-inch subgrade outfall line from the site to the canal in the event that use of the dry stream bed is unacceptable.

A closed-loop cooling system will be used, the only discharge being from occasional cooling tower blowdown. This flow, along with the boiler blowdown, is combined with the activated sludge wastewater for a total effluent flow of $100 \mathrm{gpm}$. All alcohol and gasoline storage tanks as well as the fermenters will be diked for spill containment.

\section{Solid waste}

Debris from grain handling and ash from the wood-fired boller system will be trucked off-slte for use as a soil nutrient. Accumulated solids which will be periodically dredged from the waste water treatment basin will be trucked to a land fill.

\section{Thermal}

The principal source of thermal discharge will be from the boiler/DDGS dryer system exhaust stack. The existing gases will be comprised of the products of wood fuel combustion and moisture evaporated in the DDGS dryer. The temperature of this discharge will be approximately 200$250^{\circ}$ F. Thermal radiation from process equipment will be minimal because most of the system and interconnecting piping will be insulated for operating economy and personnel 
protection. The only point of constant stream exhaust will be from the evaporator and cooking air ejector systems. The heat from these sources is negligible.

\section{Noise}

External noise is not expected to be a problem, as most of the noise-producing process equipment will be housed in the main process building. Noise emanating from the outdoor evaporator air ejector system will be muffied to a non-objectionable level.

\section{Odor}

A slight corn and DDGS oder will prevail in the area at times but is not expected to be significant. The dryer exhaust duct can be retrofitted with an absorber if the DDGS odor proves to be objectionable. The fermenters will be of enclosed-top design since they will be outdoors. prevailing winds will normally not carry unpleasant odors to nearby communities.

\section{Visibility}

Since the only exhaust points are at the boiler/DDGs dryer system stack, the dust control system, cooling tower, evaporator, cooking air ejectors, and an occasional boiler blowoff, the exhaust plumes will consist mostly of water vapor, which would have a minimal effect on local atmospheric visibility. The elevation profile of the plant will be similar to that of other chemical processing plants, with tanks, buildings, and open steel structures set as far back from the road as possible.

\section{3 HEALTH AND SAFETY CONSIDERATIONS}

The proposed plant will involve complex machinery operating at low to medium temperatures and pressures, and the handling and storage of hazardous chemicals and materials. However, the hazards in this facility are relatively well known because of long experience in the grain-processing and beverage distillery industries. With the use of proper procedures and controls, the plant will be safe and heal thful. 
Common-sense controls and standard precautions should be rigorously followed. Training will be provided for plant operators and other personnel. The following steps for training plant personnel will be taken:

- Detailed operating procedures (including safety procedures) will be documented in a technical operation manual. Detailed process diagrams or a scale model with color-coded piping illustrations will be provided. - Operators will be trained to wear hardhats and be aware of plant safety features and location of fire equipment.

- Training by the equipment supplier at the factory or on the job will be provided.

- Training by the suppliers of such materials as enzymes and yeast will be provided.

Many precautions will be taken to avoid the possibility of injury at the plant site. The training programs will cover operator procedures. The plant will be designed to incorporate safety features to minimize hazards. At a minimum the following precautions will be taken.

Hazards Precautions

Overpressurization; explosion of boiler

Scalding from steam gasket leaks

Contact burns from lines
- Regularly maintained and checked safety boiler "pop" valves will be installed, set to relieve pressure when it exceeds the maximum safe pressure of the boiler or delivery lines.

- Strict adherence to boiler manufacturer's operating procedure will be ensured.

- Continuous operator attendance during boiler operation will be required.

- Baffles will be placed around flanges to direct steam jets away from operating areas.

- All steam delivery lines will be insulated. 
Ignition of ethanol and gasoline leaks/ fumes or grain dust

Handling acids/ bases

Suffocation
- Fully enclosed explosion-proof electric motors will be used.

- All equipment will be fully grounded to prevent static electricity buildup.

- No smoking or striking of matches around ethanol tanks, dehydration section, distillation columns, or condensers will be allowed.

- Metal grinders, cutting torches, welders, etc., will not be used around systems or equipment containing ethanol. All vessels will be flushed and vented prior to. performing any of these operations.

- Protection of fumes from concentrated acids or bases will be provided.

- Goggles will be worn whenever concentrated acids or bases are handled.

- Acids or bases will not be stored in overhead work areas or equipment.

- Acids or bases will not be carried in open buckets.

- Proper materials will be used for construction for all acid or base storage containers, delivery aides, valves, etc.

- Fermenters, beer well, and stillage tanks will be properly vented. 
The design and construction of the plant will be in compliance with the following standards and codes:

Title Code*

Prevention of Dust Explosion in Industrial Plants

NFPA 63

Basic Classification of Flammable

NFPA 321

and Combustible Materials

Liquids static Electricity

NFPA 77

Flammable and Combustible

NFPA 30

Liquids Code

Occupational Noise Exposure

OSHA 191094

Machinery and Machine Guarding

OSHA

Subpart $\mathrm{O}$

Power Piping

ANSI B 31, 1

Standard for Steel Aboveground Tanks

UL 142

for Flammable and Combustible Liquids

Boiler and Pressure Vessel code ( $B \& P V$ )

ASME Code Section IV \& VII

Division I

All electrical instrumentation

NFPA 70-1978

National Electric Code

Class II

Division I

* Abbreviations :

NFPA - National Fire Protection Association

OSHA - Occupational Safety and Health Administration

UL - Underwriters Laboratory

ASME - American Society of Mechanical Engineers

ANSI - American National standards Institute 
Fire protection is also a major concern. At a minimum, the following precautions will be taken.

- Provide automatic sprinkler protection; preferably of a type designed to flood the area.

- Sprinkler control valves, dry-pipe valves, and riser drains will be readily accessible at all times to plant personnel.

- Small hoses with combination shutoff nozzles will be provided throughout the plant. Hose stream demand is a minimum of $500 \mathrm{gpm}(190 \mathrm{dm} 3 / \mathrm{min})$. for at least 60 minutes.

- Suitable portable fire extinguishers will be provided throughout the plant.

In cooperation with the Panola County Hospital, located in Sardis, Sun Belt will include first aid information and training sessions in the overall training program for plant personnel.

\subsection{SOCIOECONOMIC CONSIDERATIONS}

Sun Belt Energy Corporation is sensitive to any possible adverse socioeconomic impact that the plant may have on the local community. Our analysis indicates that panola county and the community of sardis will derive an economic benefit from the plant, and no adverse community or social impact is anticipated.

The proposed plant will create 36 full-time jobs, including 7 in management, 16 for plant operators, and 13 for unskilled labor. These positions will be filled primarily from among local residents. Unemployment in Panoia County is well over 15 percent. Two major manufacturing companies in Sardis, Roper Luggage and Poloron Products, closed down in 1981, thereby eliminating 1,200 jobs. The remaining manufacturers currently operating in Sardis are the following. 
Affiliated Hospital Products, Inc.

D.B. Floyd Lumber Company

Midsouth Gravel, Inc.

Midsouth stamping, Inc.

Mississippi Mills, Inc.

Panola County Ready Mix, Inc.

Erwin M.B. Schwabb Company, Inc.

The Southern Reporter

Coca Cola Bottling Company

Hygiene Products

Because there will be no net population growth from the proposed plant, no shortage of facilities or community services will result. Sardis has a local police and fire department, a hospital with 70 beds, a public school, churches, hotels, waterworks, and sanitary sewers. (since water will be obtained from a well on the plant site and wastewater will be treated at the site, the community water and wastewater facilities will not be affected.)

As previously described, transportation facilities are adequate for the proposed plant. Furthermore, sardis is served by 4 motor freight carriers: Beard Truck Line, J\&G Express, Shippers Express, and Yeliow Freight System. Anticipated daily truck and rail traffic to serve the plant is as follows: 13 to 15 tractor-trailer trucks to transport wood fuel and ethanol and DDGS products; and 3 to 4 railroad hopper cars per day carrying grain feedstocks.

Land use and housing stock in Panola County have been stable for over two decades. The impacts of the plant will not change the land use, and should have a small change in the number of houses in the 3-county area. 


\subsection{IMPACT ON REGIONAL ECONOMIC ACTIVITY}

The proposed plant would significantly increase economic activity in northern Mississippi. The production of ethanol and DDGS from local agricultural (corn) and forestry (wood fuel) feedstocks would stimulate secondary economic benefits in addition to the direct effect of the plant. Dr. Robert L. Williams, an economist at Mississippi State University, estimates that the economic impact of the plant on the local economy would be about $\$ 60$ million annually.*

To supplement Dr. Williams's analysis, we took a different analytical approach to identify a lower bound of economic activity. To calculate net economic effects, we used the Bureau of Economic Analysis (BEA), U.S. Department of Commerce, gross output multipliers for sector 5 (food, feed grains, and grass seeds) and sector 42 (chemicals and allied products). While sector 53 (transportation, communications, and utilities) may be affected, we assumed those benefits to be relatively insignificant because of the offsetting decrease in the use of gasoline.

The result of the analysis was a net increase in annual economic activity from the proposed plant of over $\$ 21$ million. The analysis is shown in Exhibit 5.4. For Sector 5, corn, wood, and fertilizer were assumed to be produced in Mississippi. For Sector 42, wood, corn, denaturant, electricity, and chemicals were subtracted so as to account only for the value added in this sector.

Of the \$21-million increase in economic activity, over $\$ 18 \mathrm{milli}$ ion would be in the agricultural sector (Sector 5). This would be a trementnils hnost to local farmero, who currently face financial problems. From 1950 to 1980, Mississippi's net farm income declined by 20 percent. During that time, the consumer price index advanced by 242 percent.** Furthermore, in 1981, low crop prices and high interest rates were especially damaging to Mississippi farmers.

\footnotetext{
* See Exhibits 5.2 and 5.3.

** R. Williams, "61 Percent Net Income. Decline Staggering." Delta Farm Press, August 28, 1981.
} 
In addition to economic impacts, increased corn and wood production would improve crop and forestry management in Panola and surrounding counties. In recent years, local farmers have planted soybeans as their principal crop. However, according to the Cooperative Extension Service (CES) of Mississippi State University, most of the soybean crop is infested with cyst nematodes, a problem that can be solved only by crop rotation with crops such as corn or grain sorghum. The proposed plant would provide a good market for corn and grain sorghum (milo). Furthermore, added crop residue plowed under from these crops would increase soil productivity.

In addition to acting as a local market for low-quality hardwoods, the proposed plant would assist many landowners who are attempting to convert their forest reserves to grow more efficient loblolly pine. According to Dr. Thomas A. Manahan of the Extension Forestry Department, "The harvest and utilization of much of this heretofore unmerchantable wood could significantly lower site preparation costs for landowners. If low-quality hardwood becomes an asset that can be liquidated, many landowners may be able to afford to initiate a forest management program on their land rather than do nothing."

\section{6 REGULATORY AND PERMIT REQUIREMENTS}

Sun Belt Energy Corporation is interested in minimizing any construction delays arising from regulatory or permit requirements. Accordingly, the company has held meetings with the Mississippi Department of Natural Resources and local officials to review key regulatory requirements and the proposed project schedule. Then research was done to develop the master list of regulatory requirements and to prepare a comprehensive schedule for obtaining permits. Appendix A presents a data sheet for each permit or regulation, which includes a listing of the administering agency, the agency contact and telephone number, the action that triggers the requirement, permit timing, and information requirements.

No regulatory barriers have been identified and none are anticipated. Because of the plant's small size, a prevention of significant deterioration permit from the 
U.S. Environmental Protection Agency is not needed. Furthermore, no other federal permits are required for the plant. A listing of definite regulatory and permit requirements is shown in Exhibit 5.5. Our schedule of regulatory requirements is shown in Exhibit 5.6, which indicates duration, lead times, and milestones. 
Exhibit 5.1

Population and Economic Characteristics of Panola County and Sardis, Mississippi

\section{Population}

\begin{tabular}{lrrrr} 
& \multicolumn{1}{c}{1960} & \multicolumn{1}{c}{1970} & \multicolumn{1}{l}{1975} & \multicolumn{1}{c}{1980} \\
Panola County & 28,791 & 26,829 & 27,587 & 27,579 \\
Sardis & 2,098 & 2,391 & 2,547 & 2,481
\end{tabular}

Median School Years Completed in (1970)

Panola County .............. 9.2

N. Delta ................... 8.9

Mississippi .............. 10.7

U.s. ....................... 12.1

Total Employment

(Place of Work) 1976 Panola County

Percent

Manufacturing .............2,200

26

Government ..................1,440

Services .................. 1,240

Wholesale \& Retail Trade ...... 1,320

17

15

16

Agriculture: Domestics,

Self-employed, etc. ..........1,980

Construction ............... 190

Mining ................. 80

Total ............... 8,450

$\underline{\text { Panola County Median Family Income (1969) }}$

Panola County ............ $\$ 4,982$

N. Delta Area ............ \$4,885

Mississippi .............\$6,071

U.S. .................. $\$ 9,590$

Percent of Persons Living Below the Poverty Level (1969)

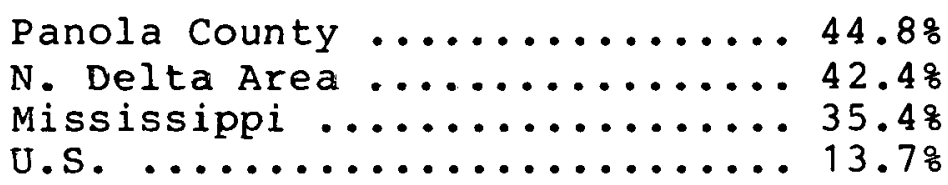


Exhibit 5.2

Economic Impact of Ethanol Plant

Prepared by: Dr. Robert L. W1lllams

ExtensLon Iconomlst

Mlsslssipl state Uaiversfty

Apr1! 1980

PLANT

3,000,000 gallong (Annual Production)

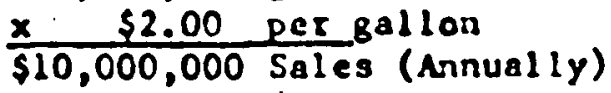

$\times 4$ (output Multipller)

$\$ 40,000,000$ - Econom1c Impact

CORS

2,000,000 Bu. (Annual plant requlrement) wlll requlre

28,571 actes of corn at 70 . Bu. per ncre.

Some of the 29,571 acres wfll come out of cottun acreage and some from soybean acreage.

The value of soybeans produced per acre is less than the value of corn produced, however, the value of cotton produced per acre is higher than the value of com produced so these are assumed to be off setting as far as the economic lowact is concerned.

The plant would uge 3000 bu. of com per day. Mbout 200,000 gallons of feed by-product will be produced daily.

When sold at $\$ 25.00$ per 1000 gallons this would generage $\$ 5,000$ income da1ly or $\$ 1,825,000$ annually.

200,000 gallons of feed-by-products produced dafly will feed about 10,000 head of beef cattle. A galn of $2.51 \mathrm{lug}$. per day wlll cause becf production to lacrease 25,000 lus. dally or 9.1 mlllions lbs. annunliy. When this is valued at 70 cents per pound the increase in beef sales in the area will be about $\$ 6,370,000$ annually.

$$
\begin{aligned}
& \$ 6,370,000 \text { (Annual Snles) } \\
& \times 3 \text { (Adjusted output Multlpllcr) } \\
& \frac{\$ 19,100,000 \text { Economlc Impact }}{\text { (m) }}
\end{aligned}
$$

Adding the economic Impact resulting from the sale of alcbhol and the sale of beef produced in the area ylelds a total Economlc Impact in the area of almost $\$ 60,000,000$ ennually. This will provide $n$ tremendous boost to the economy of the entlre stea. 
Exhibit 5.2 (continued)

\section{ECONOMY - PANOLA COUHTY}

M1solsolppl Research and Development Center 1978

Economle Development Blueprint for North Vel ta Keglon of Miss.

(Panola County Included)

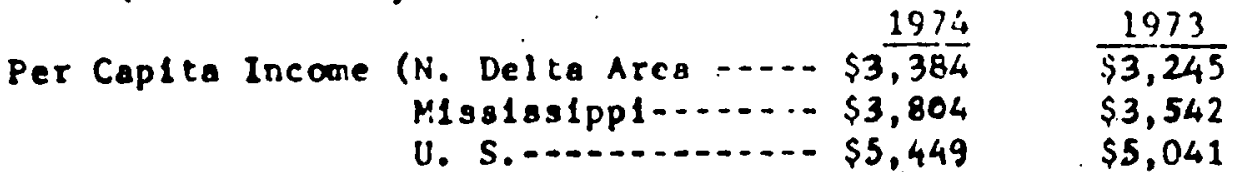

Employment Distribution in N. Delta Arca (1976)

Agriculture, Domestics \& Self Employed -

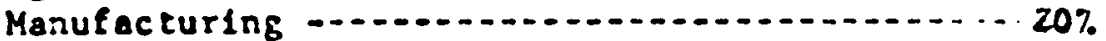

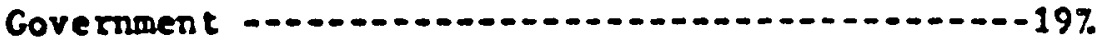

Services

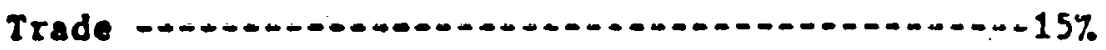

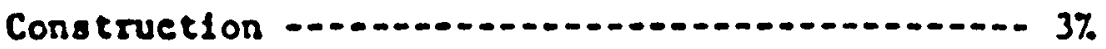

Manufacturing Employment D1stribution in the North Delta Area (1976)

Lumber \& Wood -........... $5 \%$

Furnlture \& Fixtures

Food -...................

Text1les

Apparel -.

Non-Electrlcal Machlnery -.................... $9 \%$

Socto Economle Characterlstles of Panola County

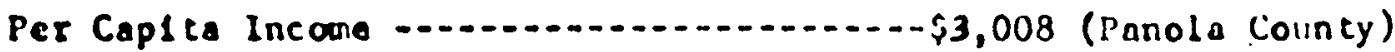

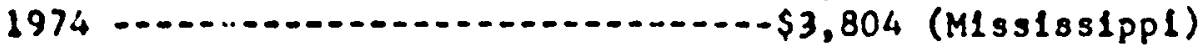

1973 County Yopulat1on

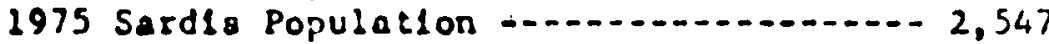

Cood rall and Interstate connections make Panola County attractive to new industry. The county has a particular need for eddltional manufacturing jobs for con.

In $1978 \mathrm{R}$ \& D cstimated $42 \%$ of houslng in l'anola county ls sujstandard. Panola County Medlan Famlly Incone (1969)

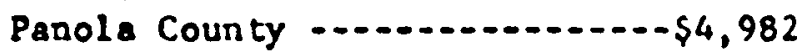

N. Delta Area $\ldots . . . . . . . .54,885$

Mississlppl ................. $\$ 6,071$

U. 5.

Percent of Persons living below the poverty levels (1969)

Panole County -...............-44.87.

N. Delta Area

M1salsslppl

U. S. 
Total Employrient - Place of liealdence - $197 t$

Panola County -.................... 9.150

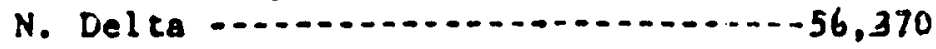

Total Employment (Place of Work) 1976 Panoln County

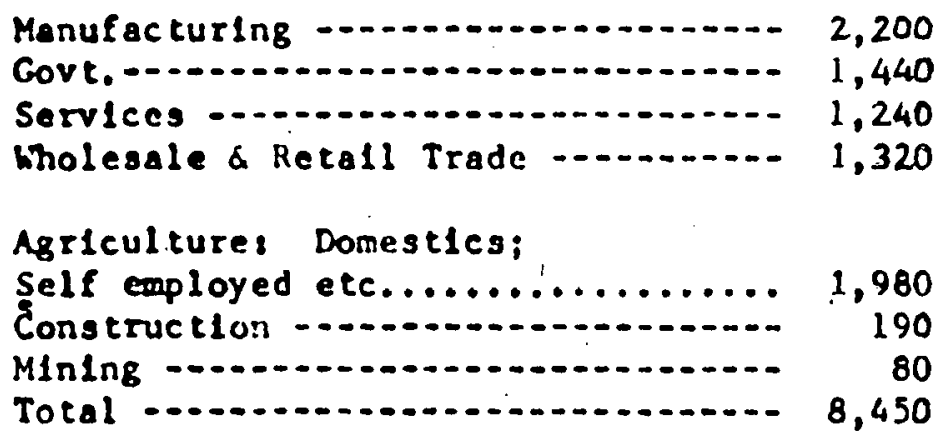

Populatlons

$\begin{array}{lrrrr} & 1960 & & 1970 & \frac{1975}{1} \\ \text { Panola rounty } & 28,791 & 26,829 & 27,587 \\ \text { Sardis } & 2,098 & 2,391 & 2,547\end{array}$

Median School Years Completed in (1970)

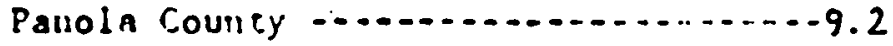

N. Delta $-\ldots .0-1.9$

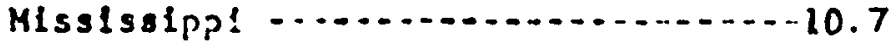

U. S. 
(Miosisslppi Crop \& Livestock Reporting Serilce)

PANOL.A. COUNTY

$\begin{array}{lrr} & \text { Acres } & \text { Y1eld/icre } \\ \text { Soybeans } & 112,500 & 20.4 \text { (Bu.) } \\ \text { Cotton } & 30,400 & 557 \text { (Lbs.) } \\ \text { Wheat } & 4,200 & 28.3 \text { (Bu.) } \\ \text { Com } & 1,100 & 76.0 \text { (Bu.) } \\ & \text { Number } \\ \text { Beef Cows } & & \\ \text { Hogs o Plgs } & 26,000 & \end{array}$

PANOLA COUNTY ACRICULTURAL STATISTICS (1974 CenSUS)

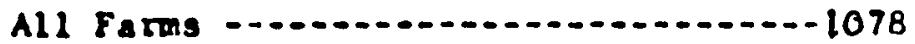

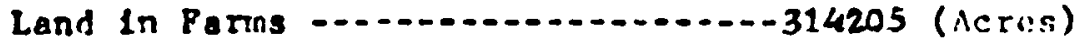

Average slze of Farm .......... 291 (Acres)

Total Cropland -.................-184266 (Acres)

Woodland -.......................... 51573 (Acres)

Value of AR. Prodicts Sold
(A) Per Farw $\ldots \ldots \ldots \ldots+16858$ dollars
(B) Crops ......................572,000 dollars
(C) Foregt Products …...... 14,000 dollars
(D) Livestock ............. 3,587,000 dollars

Cattle \& Calves ................ 47,083

llogs \& Plgs ........................... 3,349

Pull daner fartig …........ 734

Part Ownet Famos ....... 236

Tenant Parms ............................ 108

\section{Farm Production Expenses}

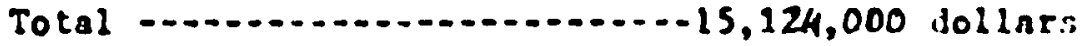

Feed Purchased for Livestock---- - 1, 186,000 dollara Hlxed Formula Feeds ........... 772,000 dollars

Gasoline other Petroleum Fuel

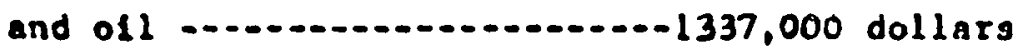

Value of Products Sold (Total).....-18,173,000 dollars CROPS HARVESTED $9(1974)$

Corn -.-Farms -... 145

Acres -- - -1021

Bu. ......-33918 
Exhibit 5.2 (continued)

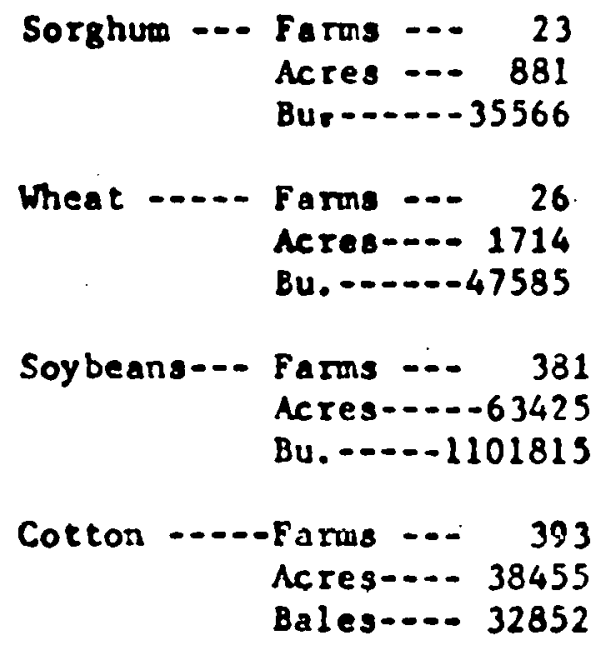

Grain Storage Facllitles

1,000 Bu. \& Over -........-.55 (Fams)

Fattened Cattle Sold

Farms - - $-0 .-0-0-8$

Cattle $\cdots \cdots \cdots-. . \cdots-345$

1970 POPULATION (MAFES BULLETIN 809)

Total $\ldots \ldots \ldots-26,829$

White $-\ldots-\ldots-13,061$

Non-whl, e- $\ldots-\ldots-13,768$

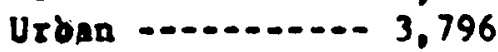

Rural-Non Parm-- $-18,495$

Farm

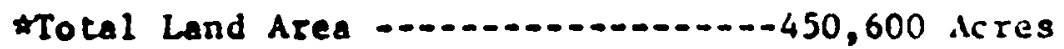

Comerical Forest_................123,500 Acres

Growlng Stock Volume on Commerlcal lorest Land by Specles Croup - 1973

$55,600,000$ Cublc Feet 


\title{
Mississippi Research \& Development Center
}

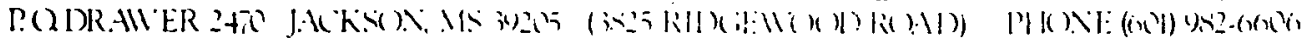

March 15, 1982

Mr. Billie E. Shepard, President

Sun Belt Energy Corporation

212 North Main Street

Sardis, MS 38666

Dear Mr. Shepard:

Per your request, I have reviewed the employment impact statement of a proposed power alcohol plant located in Panola County, and have estimated increased Mississippi tax revenues from the proposed power alcohol plant. Time limftations prevent a review of supporting materials provided by the Bechtel study and Dr. Robert Williams of Mississippi State University relative to the tax revenues. Therefore, the economic impact provided by your study of $\$ 2,452,500$ in additional employment dollars was used to calculate additional tax revenues.

The employment multiplier of 2 is valid based on present employment ratios in Mississippi. Estimating the number of additional agricultural workers needed to supply the plant with grain is a difficult undertaking. Assuming that this grain production is in excess of present agricultural production, the creation of 170 additional related jobs appears to be feasible.

Assuming an increase in payrolls of $\$ 2,452,500$ as a direct result of the proposed power plant and an income multiplier of 1.7 , the total increase in personal income would be $\$ 4,169,250$. A publication by the U. S. Bureau of the Census, "Government Finances in 1979-80," indicates that for every increase of $\$ 1,000$ in personal income in Mississippi, state and local tax revenues increase approximately $\$ 100$. An increase of $\$ 4,169,250$ in personal income would result in an increase of $\$ 416,925$ in state and local taxes.

The above estimates of an additional $\$ 416,925$ in state and local taxes collected in Mississippi would come from direct wages and salaries paid to workers employed by the development of a power alcohol plant. The above figure does not include the total economic impact of $\$ 70$ million as presented in Dr. Williams' study. From this $\$ 70$ million, additional state and local taxes would be generated.

Sinçerely,

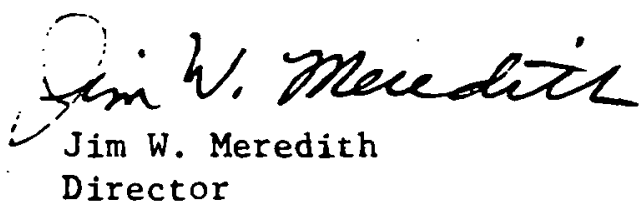

$\mathrm{JWM} / \mathrm{kh}$ 
Exhibit 5.4

Annualized Increase in Economic Activity

Economic Activity

( $\$$ millions)

\begin{tabular}{lr} 
Sector 5* \\
\hline Corn & $\$ 4.75$ \\
DDG & $\begin{array}{l} \\
\text { Woỏd }\end{array}$ \\
Fertilizer & 0.43 \\
Sum & 0.06 \\
Multiplier & 7.95 \\
$\quad$ Total Sector 5 & 2.10 \\
\hline 18.12
\end{tabular}

Sector $42 *$

Ethanol

wood

$\$ 8.50$

corn

$(0.43)$

$(4.75)$

Denaturant (gasoline)

$(0.22)$

Chemicals.

$(1.25)$

Electricity

$(0.33)$

Sum

1.52

Multiplier

Total sector 42

$\frac{2.00}{\$ 3.04}$

Total Increase in Economic Activity

$\$ 21.16$

* Bureau of Economic Analysis, U.S. Department of Commerce, sectors for economic activity multipliers. 
Exhibit 5.5

Regulatory Requirements

\section{Before Construction}

Air Emission and Wastewater Control Permit Approval of Location and Construction of Water Works

Site Approval of Wastewater Treatment Facility

\section{Before Cperation}

Compliance with OSHA

Compliance with Noise Fegulations

Certificate for Boilers

Septic System Permit

Approval of Potable water Supply

\section{$\underline{\text { Agency }}$}

Mississippi Department of Natural Resources

Mississippi Department of Natural Resources

Mississippi Department of Natural Resources

\section{Agency}

U.S. Department of Labor

Mississippi Department of Natural Resources

Mississippi Department of Labor

Mississippi state Board of Health

Mississippi state Board of Health 
Exhibit 5.6

Schedule for Regulatory Requirements

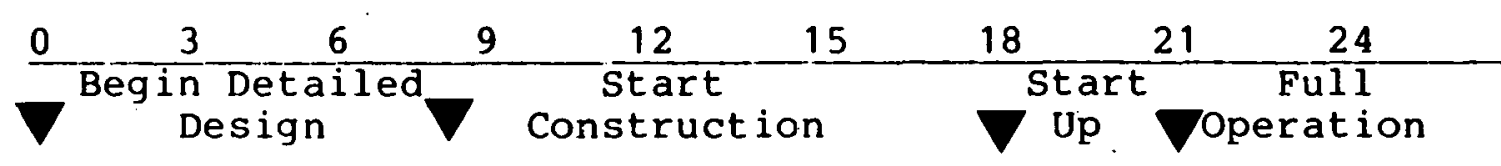

Air Emissions and

Wastewater Control Permits

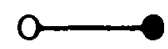

Approval of Location

of Water Works

Site Approval of

Treatment Facility

Certificate for Boilers

Septic system Permit

Approval of Potable Water Supply

$\underline{\text { Key }}$

- Application Submitted

- Permit Approved 
Good management is vital to a fuel ethanol project's success. It relies on thorough analysis, planning, and clear communication. Sun Belt Energy Corporation will provide these important ingredients. We have performed a detailed analysis of factors that are important to implementation of this project. With the assistance of Bechtel Petroleum, Inc., and Resource Planning Associates, Inc., two firms with extensive experience in designing, planning, and managing major energy projects, we have prepared a detailed project schedule, a project organization structure, and a discussion of key management activities, each of which is described in this section.

\section{1 SCHEDULE FOR \\ PROJECT IMPLEMENTATION}

The Sun Belt fuel ethanol plant would be the ninth plant constructed in the United states using the ACR process. Six plants using the ACR process are now in operation. Therefore, the critical activities in construction and their timing are well known to the ACR Process Corporation and Bechtel pctroleum, Inc. (Rechtel has designed the most recent fuel ethanol plant using the ACR process.)

Our project schedule, shown in Exhibit 6.1 , is based on that experience and allows for contingencies. The exhibit depicts major project implementation activities, from the project feasibility study phase to plant operation. The total schedule covers 36 months, from the start of the feasibility study (July 1981) to full plant operation (July 1984). The implementation activities are grouped into four categories: (1) Preconstruction; (2) Detailed Design, Procurement, and Construction; (3) Regulatory; and (4) Plant Operation. The activities in each category are described below. 
Preconstruction Activities

Three major activities require management attention prior to detailed engineering and construction. These activities. are:

- Conduct project feasibility study

- Develop community awareness and support

- Secure equity and long-term financing.

The schedule for these activities covers 14 months. As part of the feasibility study, sun Belt completed an initial financial analysis of the project in January 1982. That analysis was sufficiently positive that sun Belt began to arrange for equity and long-term financing for the project. The schedule allows 4 months after the feasibility study for financing prior to the start of engineering.

A great deal of work has been and will continue to be done to publicize the project and its potential benefits to the community. Sun Belt has received substantial support from community leaders in Sardis and Panola county. Sun Belt intends to maintain its public relations program throughout project implementation.

Detailed Design, Procurement, and Construction

The schedule for this group of activities covers 20 months. The first 2 months would be spent in preparing and finalizing contracts, first with the construction management firm and then with the engineering firm and the construction contractor. Detailed engineering would then begin, covering a period of 12 months. During the first several months, specifications for major equipment would be prepared for procurement items requiring a long lead time. The ACR process equipment would be ordered first, followed by the boiler, DDGs drying equipment, and wastewater treatment equipment. After 4 months of detailed engineering, other equipment would be ordered, including grain handling and receiving equipment, pumps, cooling tower, and miscellaneous equipment. Site clearing and preparation would be started after 8 months of

- detailed engineering. Foundations would then be installed, and the remainder of the plant would be built over a period of 9 months. 
Regulatory Activities

The major regulatory activity requiring management attention will be to obtain permits for air and water pollution control. Applications will be prepared and filed with the Mississippi Department of Natural Resources (MDNR). Discussions with MDNR indicate that the permit approval process should take about 30 to 60 days; however, we have allowed over 120 days. Site inspections will also be required by the MDNR, the state Board of Health, and the Mississippi Department of Labor, for the wastewater treatment systems and the boiler after installation.

\section{Plant Operation}

Three major activities will be required before full operation of plant. Prior to long-term financing, contracts with feedstock suppliers and ethanol and DDGS customers will be executed. Sun Belt has already initiated discussions with these suppliers and buyers. The next activity is to search for, hire, and train plant operating personnel. The plant manager and engineer will be hired 8 months before plant start-up. They will assist in the recruitment of other supervisory and technical personnel to be on board 2 months prior to start-up. As part of his turnkey agreement, the general contractor will manage the process of recruiting all plant personnel. The entire plant staff will begin training and orientation 2 months before plant start-up.

Plant start-up will cover a period of about 3 months. During that period, the plant will operate 24 hours a day and seven days a week. Based on the experience of the plants now operating, we expect production to reach 90 percent of full capacity within the first 2 months, and 100 percent capacity by the end of the third month.

Our schedule represents a fast-track, but achievable, set of activities from the start of detailed engineering through the completion of construction. This is facilitated by long-lead procurement of important equipment and by close coordination among the engineering, process supplier, and construction firms. Coordination is provided by a centralized management organization with the assistance of a construction management firm. The project organization and management strategy are described in the remainder of this section. 


\subsection{PROJECT ORGANIZATION}

Sun Belt Energy Corporation will use a tightly knit management structure and will rely on highly qualified individuals in the key technical and management functions.

The proposed management organization for the construction period is shown in Exhibit 6.2. The Sun Belt Energy Corporation President, Mr. Billie E. Shepard, will retain final decision-making authority. In the construction phase of the project, he will be supported by an independent construction management firm with special expertise in construction cost control and scheduling. The engineering firm and general contractor will be selected on the basis of experience, quality assurances, and cost. Planning for maximum operating revenues (e.g., ethanol and DDGS contract negotiations) and reduced operating costs (e.g., grain supply contract negotiations, co-op storage participation), and assistance in financing implementation will be provided by Resource Planning Associates, Inc. The process supplier will be the ACR Process Corporation.

The responsibilities for key construction activities are shown in Exhibit 6.3. The construction management firm will facilitate coordination among the team members, since each activity will involve more than one firm.

The type of contract used for engineering and construction will be important in managing the construction process. Where possible, Sun Belt will use fixed-price contracts for engineering and construction services. Warranties and other contract assurances for construction timeliness, quality, and process efficiencies will be required.

Several months prior to start-up, the management organization for operating the plant will be formed. This management structure is shown in Exhibit 6.4. The plant personnel requirements for operation are shown in Exhibit 6.5, which includes the necessary personnel for continuous 24-hour operation of the plant. Approximately 36 persons will be required to maintain full production ( 3 shifts per day). The plant manager will be responsible for the overall operation of the plant and will report to the president of Sun Belt. The shift supervisors, plant engineer, and controller will report to the plant manager. The shift supervisors will be directly responsible for the day-to-day operation of the plant. The plant engineer 
will be responsible for maintenance and plant performance, using a maintenance and utility crew 3 shifts per day. A laboratory technician will be responsible for maintaining a quality assurance testing program and will report directly to the plant engineer. Services of an independent testing laboratory will be used for product quality and certifications as required.

The plant manager and engineer will be on staff 8 months before plant construction is completed. Two months before plant start-up, shift supervisors and other managerial personnel will be on staff for orientation and training. The purpose of early staffing is to minimize start-up time and costs. The more familiar the personnel are with the systems and operations of the equipment, the more efficiently and quickly the plant can be brought to full production capacity. The plant will be staffed by well-qualified personnel with chemical process industry experience.

\subsection{KEY MANAGEMENT ACTIVITIES}

Sun Belt management's aim is to focus on the project elements that are most important to project construction cost control and operating revenue enhancement. Although this seems an obvious goal, it is often difficult for management to identify and concentrate attention on the most important issues. Since the managers' time will be limited, given the day-to-day activities in the construction phase of the project, it is critical that they focus only on the essential issues; other issues can be easily delegated.

Based on an analysis of factors that will affect the project's financial performance, and on interviews with other fuel ethanol plant owners and constructors, sun Belt has carefully identified the elements that are key to project implementation and has an understanding of their relative importance. These issues and our approach for handling them are described below.

- Prices for products and feedstocks. Although it has no control over market prices for corn or wood feedstocks or ethanol prices, Sun Belt intends to sign 
contracts with feedstock suppliers and buyers of its products that will minimize sharp price fluctuations over a specified period. For grain, wood purchases, and DDGs sales, the contracts will have prices tied to gross economic indicators. For ethanol sales, we have structured contracts with prices tied to market prices of unleaded regular gasoline.

- Long-term financing. Significant management attention will be placed on securing long-term financing for the project.

- State permit approvals. Sun Belt will be keenly responsive to the requirements for obtaining air and water pollution control permits from the state of Mississippi, and will diligently prepare the permit applications and closely monitor them through the MDNR approval process.

- Construction schedule and equipment delivery. Sun Belt will retain a well-qualified construction management firm to monitor the construction schedule closely and to expedite delivery of important plant equipment. To encourage timeliness, payments for services will be based on percent completion.

- Plant performance. Plant performance will depend on two factors, equipment performance and operator performance. Equipment performance will be assured by guarantees provided by the process supplier, ACR Corporation, and by the design engineering firm. Sun Belt will provide an extensive training program for the plant operators, which will include training by the equipment vendors and the process suppliers. Sun Belt also intends to fully meet all occupational Health and Safety Act requirements for operational procedures and workers' safety.

- Detailed engineering quality and schedule. Sun Belt's contract for detailed engineering services will be fixed-price with payments tied to deliverables. Furthermore, Sun Belt intends to engage an engineering firm that has substantial experience in designing fuel ethanol plants. Bechtel Petroleum, Inc., would be a candidate for this project, as would other firms of a similar caliber.

- Cash flow management. Sun Belt will use its financial analysis model to plan and control the cash flows during plant construction and operation. Financial management will be a discrete function that will 
receive the highest level of sun Belt management's attention.

- Construction cost. In the first instance, construction cost will be controlled by the use of wellestablished cost-estimating techniques prior to the start of construction. The engineering firm will prepare the initial estimates, which will then be checked by the construction management firm. Costs will be further controlled by using a fixed-price turnkey contract. Change orders that may result in additional costs will be carefully reviewed by the construction management firm and by sun Belt before approval.

- Personnel hiring and training. Sun Belt recognizes that the success of the project will be a function of the quality of its personnel. Sun Belt will provide sufficient compensation to attract people with outstanding capability and experience. 
Exhibit 6.1

Schedule of Major Project Implementation Activities

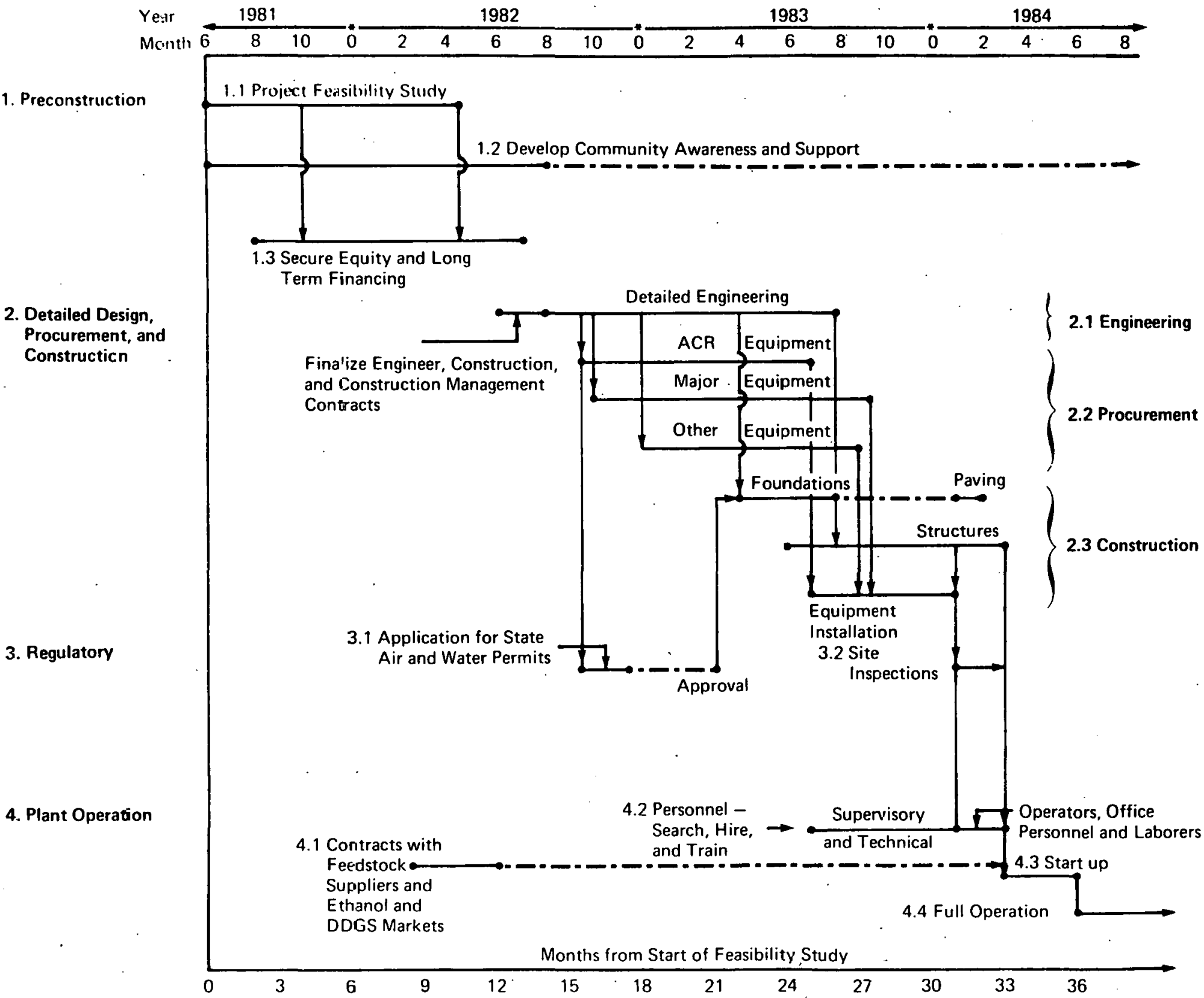




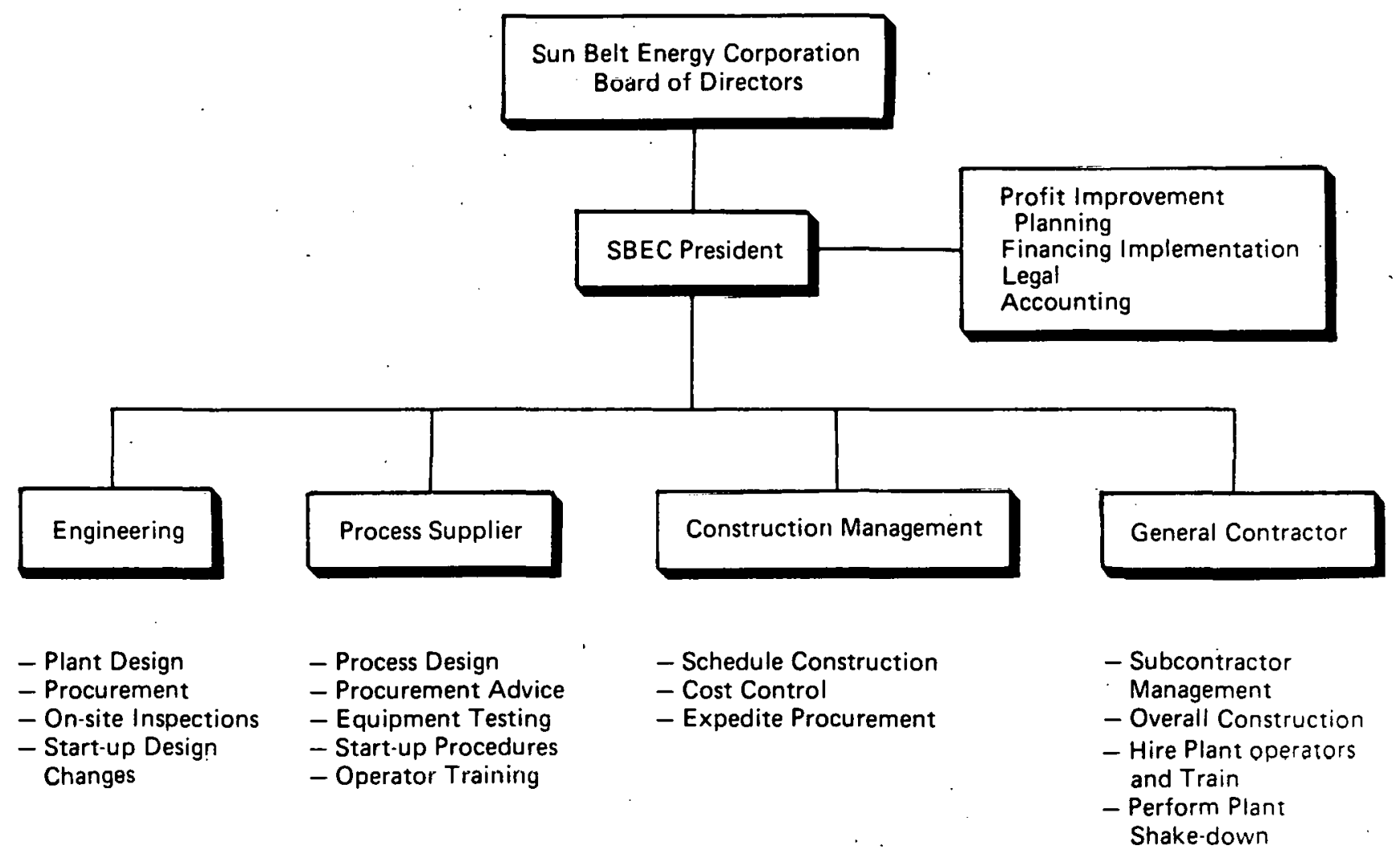


Exhibit 6.3

Responsibilities for Key Construction Activities

Key Construction Activities

\begin{tabular}{|c|c|c|c|c|c|c|c|}
\hline Team Member & $\begin{array}{l}\text { Plant } \\
\text { Design }\end{array}$ & $\begin{array}{l}\text { Engineering } \\
\text { Process } \\
\text { Equipment }\end{array}$ & Procurement & $\begin{array}{l}\text { Expedite } \\
\text { Procurement }\end{array}$ & Construction & $\begin{array}{l}\text { Initial } \\
\text { Start-up }\end{array}$ & Other \\
\hline $\begin{array}{l}\text { Encineering } \\
\text { Firm }\end{array}$ & $\begin{array}{l}\text { Detailed } \\
\text { Engineering }\end{array}$ & $\begin{array}{l}\text { General } \\
\text { Specifications }\end{array}$ & $\begin{array}{l}\text { Initiate } \\
\text { Procurement }\end{array}$ & $\begin{array}{l}\text { Maintain } \\
\text { Contact } \\
\text { with Vendor }\end{array}$ & $\begin{array}{l}\text { Field } \\
\text { Inspection }\end{array}$ & Monitor & $\begin{array}{l}\text { Assist } \\
\text { Obtaining } \\
\text { Necessary } \\
\text { Permits/ } \\
\text { Design } \\
\text { Changes }\end{array}$ \\
\hline $\begin{array}{l}\text { Coristruction } \\
\text { Maragement } \\
\text { Firn }\end{array}$ & $\begin{array}{l}\text { Review } \\
\text { Cost and } \\
\text { Schedule } \\
\text { Revisions }\end{array}$ & & $\begin{array}{l}\text { Maintain } \\
\text { Schedule/ } \\
\text { Cost Control }\end{array}$ & $\begin{array}{l}\text { Expedite } \\
\text { Delivery/ } \\
\text { Monitor } \\
\text { Cost }\end{array}$ & $\begin{array}{l}\text { Monitor/ } \\
\text { Review } \\
\text { Change } \\
\text { Orders }\end{array}$ & . & $\begin{array}{l}\text { Consult on } \\
\text { Development of } \\
\text { Engineering } \\
\text { and Construction } \\
\text { Contracts }\end{array}$ \\
\hline $\begin{array}{l}\text { Equipment } \\
\text { Vendors }\end{array}$ & . & $\begin{array}{l}\text { Work with } \\
\text { Process } \\
\text { Supplier }\end{array}$ & $\begin{array}{l}\text { Fabricate } \\
\text { Equipment }\end{array}$ & $\begin{array}{l}\text { Expedite } \\
\text { Delivery }\end{array}$ & & . & \\
\hline $\begin{array}{l}\text { Process } \\
\text { Supp:lier }\end{array}$ & $\begin{array}{l}\text { Consult on } \\
\text { Process } \\
\text { Requirements } \\
\end{array}$ & $\begin{array}{l}\text { Detailed } \\
\text { Specifications }\end{array}$ & $\begin{array}{l}\text { Review } \\
\text { Process } \\
\text { Equipment } \\
\text { Orders }\end{array}$ & $\begin{array}{l}\text { Maintain } \\
\text { Contact } \\
\text { with } \\
\text { Vendor }\end{array}$ & $\begin{array}{l}\text { Inspect } \\
\text { Equipment } \\
\text { Installation }\end{array}$ & $\begin{array}{l}\text { Develop } \\
\text { Operating } \\
\text { Procedures } \\
\text { and Consult }\end{array}$ & $\begin{array}{l}\text { Assist in } \\
\text { Pre-operation } \\
\text { Training }\end{array}$ \\
\hline $\begin{array}{l}\text { Gene-al } \\
\text { Contractor }\end{array}$ & $\begin{array}{l}\text { Consult on } \\
\text { Site Engir.eering }\end{array}$ & & & . & $\begin{array}{l}\text { Build Plant/ } \\
\text { Manage } \\
\text { Subcontractors }\end{array}$ & $\begin{array}{l}\text { Hire Operators } \\
\text { Run Shake- } \\
\text { Down Under } \\
\text { Turnkey } \\
\text { Agreement }\end{array}$ & $\begin{array}{l}\text { Prepare } \\
\text { As-built } \\
\text { Drawings }\end{array}$ \\
\hline
\end{tabular}




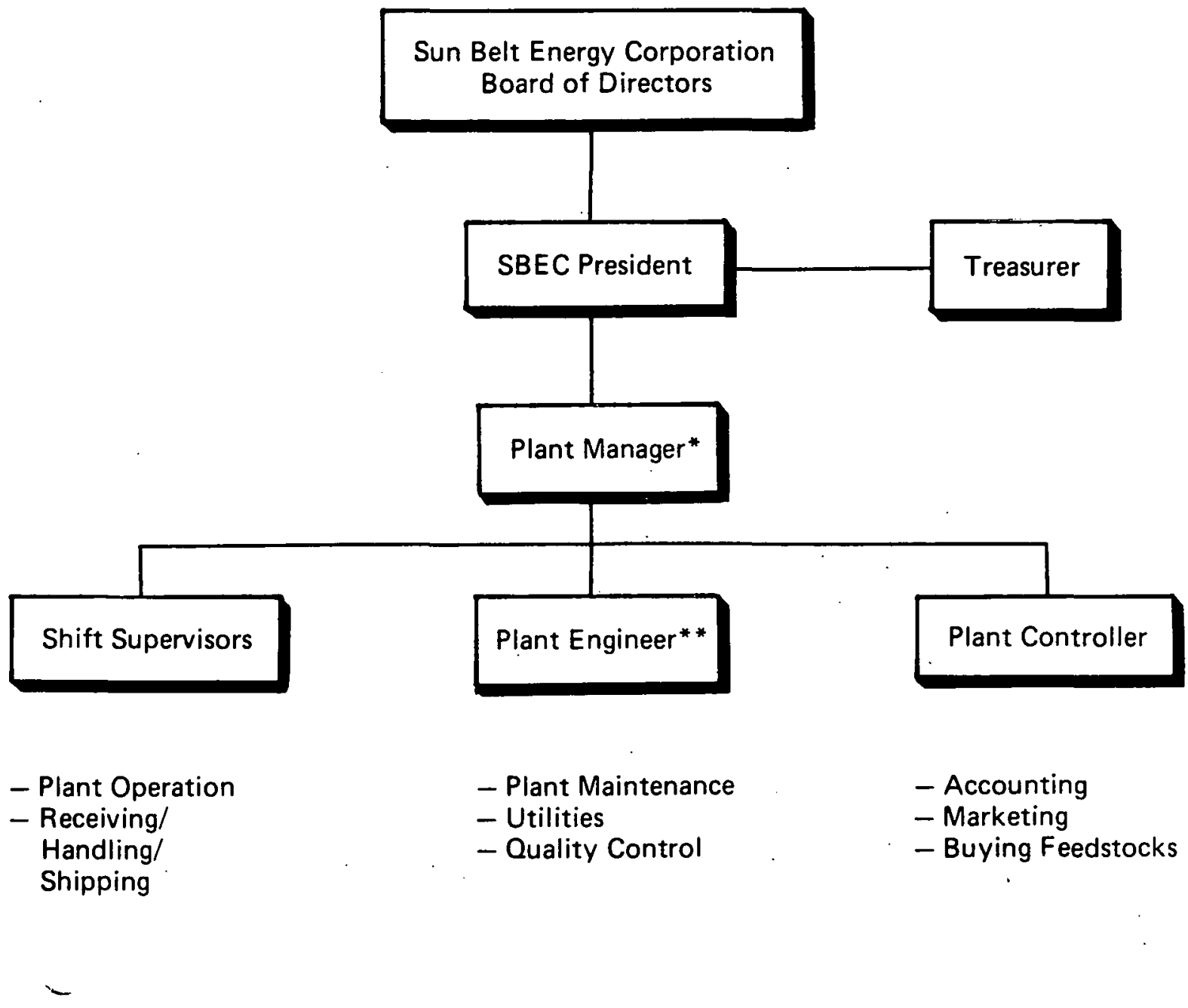

* Tlie plall manager will be qualified to fill in for the plant engineer or a shift supervisor when vacation or sickness occurs.

* The plant engineer will be qualified to fill in for a shift supervisor when vacation or sickness occurs. 
Exhibit 6.5

Sun Belt Ethanol Plant

Personnel Reguired

Persons Shift/Day Total

ADMINISTRATION

Plant Manager

General--Typist, Accounting,

Payroll, Buying, Receiving

Feedstock Procurement

DDGS/Alcohol Sales

SUBTOTAL

1

1

1

1

1

3

SUPERVISION

Plant Engineer

Plant Controller

Shift Supervisor

SUBTOTAL

OPERATIONS

Control Room

Operators

Grain Handling/Alcohol

Shipping

Laboratory

Maintenance

Utility

Security and Service

SUBTOTAL

TOTAL

$\begin{array}{lll}1 & 1 & 1 \\ 1 & 1 & 1 \\ 1 & 3 & \frac{4 \star}{6}\end{array}$

2

3

8*

2

1

3

8*

1

1

4 *

4 *

1

$\frac{1}{27}$

36

* Includes additional persons for the swing shift. 
Appendix A

PERMITS REQUIRING MANAGEMENT ATPENTION 
Permit/Regulation:

Obtain permit to construct a facility that will discharge waste into the atmosphere or any stream.

Administering Agency:

MS Department of Natural Resources

Bureau of Pollution Control

Agency Contact:

Conald Scharr

Agency Telephone:

$961-5159$

Triggering Action:

Fill out application - usually no problem, if you comply with EPA regulation.

Permit Timing:

Information Requirements:

Comments : 
Permit/Regulation:

Approval of Potable water supply

Administering Agency:

MS State Board of Health

Jackson, MS

Agency Contact:

Mr. Redding

Agency Telephone:

$\underline{354-6616}$

Triggering Action:

No strict laws - on an industry with 25 or more employees the agency checks once a year on water. Best bet would be to hook up with Como, MS water supply. If other source is required, a registered engineer would have to do preliminary work. The Board would then review and approve.

Permit Timing:

$\underline{2-3 \text { weeks }}$

Information Requirements:

Comments : 
Permit/Regulation:

Obtain state permit to operate a waste water treatment facility from which no discharge occurs.

Administering Agency:

MS Department of Natural Resources

Bureau of Pollution Control

Agency Contact:

Donald Scharr

Agency Telephone:

961-5159

Triggering Action:

Fill out application

Permit Timing:

$30-60$ days

Information Requirements:

Comments: 
Permit/Regulation:

Qualify as an interstate fuel user.

Administering Agency:

Motor Vehicle Comptroller

Motor Fuel Tax Section

Jackson, MS 39205

Agency Contact:

Jay Duckworth - Supervisor

Agency Telephone:

$601-354-7447$

Triggering Action:

$\$ 1.000$ motor fuel permit tax surety bond is required.

Permit Timing:

Two weeks after submitting surety bond.

Information Requirements:

Comments: 
A. 6

ADDITIONAL PERMITS/REGULATIONS 
Permit/Regulation:

Provide workmen's compensation insurance or qualify as a selfinsured compnay.

Administering Agency:

Workmen's Compensation Commission

POINTER INSURANCE AGENCY

Main Street - Como, MS 38619

Agency Contact:

Monroe Pointer

Agency Telephone:

- $601-526-5101$

Triggering Action:

Policy can be written when estimated payroll given by iob description is given. He sees no problems with getting an okay.

Permit Timing:

Can call Jackson and ter an okay when information reaches him.

Information Requirements: 
Permit/Regulation:

Obtain copies of the solid waste law and regulations

Administering Agency:

MS State Board of Health

Jackson, MS

Agency Contact:

C. Burks

Agency Telephone:

$\underline{961-5171}$

Triggering Action:

Copies are at printers and will not be back until first of April. They have adopted the EPA regulations.

Permit Timing:

A few days.

Information Requirements:

Comments: 
TAX

\section{PERMIT/REGULATION DATA FORM}

A. 9

Permit/Regulation:

Obtain County License and Pay Tax

Administering Agency:

Panola County Tax Collector

215 Pacahontas

Sardis, MS 38666

Agency Contact:

Pat Holley

Agency Telephone:

$601-487-2641$

Triggering Action:

Go to office and license will be issued - it is paid yearly.

Permit Timing:

30 minutes

Information Requirements:

Comments: 
Permit/Regulation:

License Trucks

Administering Agency:

Panola County Tax Collector

215 Pocahontas

Sardis, MS 38666

Agency Contact:

Pat Holley

Agency Telephone:

601-487-2641

Triggering Action:

Present titles, etc., paid yearly

Permit Timing:

$30-45$ minutes

Information Requirements:

Comments : 
Permit/Regulation:

Tax Payments

Administering Agency:

Mississippi State Tax Commission

Revenue Department

Agency Contact:

Jerry Miles

Agency Telephone:

$601-354-6274$

Triggering Action:

Annual Corporate Tax Return

Permit Timing:

Information Requirements:

Comments: 


\section{Appendix B}

COMPUTER MODEL SENSITIVITY RESULTS 
FINANCIAL ANALYSIS OF SUN BELT ENERGY CORFORATION PROJECT

CORN -TO-ETHANOL FLANT

4.8 MIL GFY RATEI CAPACITY

ASSUMF:TIONS (1982\$)

14235 GPD RATED CAP

$100 \%$ ACT CAP

333 DAYS PER YEAR
DATE OF ANALYSIS:

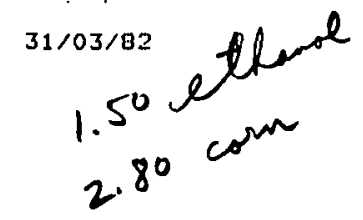

FUEL: WOOD

$$
2.8^{0}
$$

2.5 GAL ETH/BU

18 LBS DDG/BU

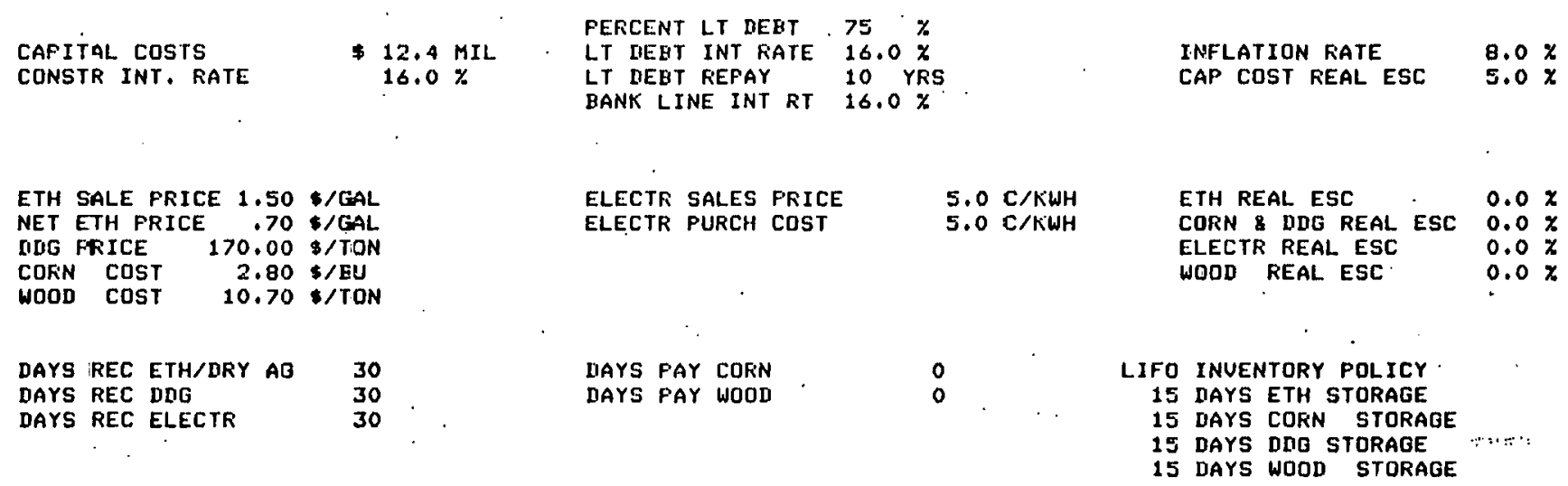

$\begin{array}{lllr}\text { FED INC TAX } & 46.0 \% & \text { EQUIP DEPR LIFE } & 5 \% \\ \text { STATE INC TAX } & 4.0 \% & \text { EQUIP ITC } & 20 \% \\ \text { STATE SALES TAX EXEMPT } & 0.0 \% \text { C/GAL } & \text { BLDG ITC } & 0 \% \\ \text { STATE GASOLINE EXCISE TAX EXEMPT } & 4.0 \text { CIGAL } & \end{array}$

STATE GASOLINE EXCISE TAX EXEMPT. 4.0 C/GAL

FED GASOLINE EXCISE TAX EXEMFT 1.0 C/GAL

RESULTS

ROE $13.1 \%$

NFU ( $25 \%$ DISC RATE)

$-3.3 \mathrm{MIL}$

FAYBACK

1793

DEET COU:

1.0 IN OFER YR 1

1.5 IN OPER YR 6

ENG cosTs $.6 \mathrm{MIL}$

INITIAL IIEBT \$12.4 MIL

MAXIMUM CASH EQUITY

$0.0 \mathrm{MW} \mathrm{ELECT}$

ANNUAL SALES:

4.3 MIL CALS ETH

16649 TONS DDG

29273 ACRES CORN 166 MIL LE STEAM
ANNUAL PLANT REEQS AT F!JLL CAFACITY

$$
\begin{aligned}
& 1.9 \text { MIL BU COFN } \\
& 6 \text { MIL KWH }
\end{aligned}
$$

16049 HEAL CATTLE 


\begin{tabular}{|c|c|c|c|c|c|c|c|c|c|}
\hline REVENUES & 1982 & 1983 & 1984 & 1985 & 1986 & 1987 & 1988 & 1989 & 1990 \\
\hline $\begin{array}{l}\text { ETHANOL } \\
\text { DIG }\end{array}$ & $\begin{array}{l}0.0 \\
0.0\end{array}$ & $\begin{array}{l}0.0 \\
0.0\end{array}$ & $\begin{array}{l}0.0 \\
0.0\end{array}$ & $\begin{array}{l}7.0 \\
3.8\end{array}$ & $\begin{array}{l}7.7 \\
3.9\end{array}$ & $\begin{array}{r}10.5 \\
4.2\end{array}$ & $\begin{array}{r}11.3 \\
4.5\end{array}$ & $\begin{array}{r}12.2 \\
4.9\end{array}$ & $\begin{array}{r}13.2 \\
5.2\end{array}$ \\
\hline DRY AGENT & 0.0 & $c .0$ & 0.0 & .3 & .4 & .4 & .4 & .4 & .5 \\
\hline ELECT PWR & 0.0 & $c .0$ & 0.0 & 0.0 & 0.0 & 0.0 & 0.0 & 0.0 & 0.0 \\
\hline TOTAL FEEUS & 0.0 & $c .0$ & 0.0 & 12.9 & 13.9 & 15.0 & 16.2 & 17.5 & 18.9 \\
\hline VAR IARLE COST & & & & & & & & & \\
\hline FEEDSTOCK & 0.0 & c.o & 0.0 & 6.7 & 7.2 & 7.8 & 8.5 & 9.1 & 9.9 \\
\hline $\begin{array}{l}\text { WOOD } \\
\text { DRY AGENT }\end{array}$ & 0.0 & C.o & 0.0 & .5 & .5 & .0 & .6 & .7 & .7 \\
\hline $\begin{array}{l}\text { DRY AGENT } \\
\text { ELECT }\end{array}$ & $\begin{array}{l}0.0 \\
0.0\end{array}$ & $\begin{array}{l}c .0 \\
c .0\end{array}$ & 0.0 & .3 & .4 & .4 & .4 & .4 & .5 \\
\hline OTHER & 0.0 & $c .0$ & 0.0 & .0 & $: 7$ & $: 4$ & .3 & .9 & .9 \\
\hline TOTAL VC & 0.0 & c.o & 0.0 & 8.5 & 9.2 & 10.0 & 10.8 & 11.6 & .12 .5 \\
\hline FIXED COSTS & & & & & & & & & \\
\hline $\begin{array}{l}\text { LABDR } \\
\text { DEPREC }\end{array}$ & & & 0.0 & .9 & 1.0 & .1 .1 & 1.2 & 1.3 & 1.4 \\
\hline$G \& A$ & $\begin{array}{l}0.0 \\
0.0\end{array}$ & $\begin{array}{l}0.0 \\
4.0\end{array}$ & $\begin{array}{l}0.0 \\
0.0\end{array}$ & 2.5 & 3.6 & 3.4 & 3.4 & $\begin{array}{r}3.4 \\
5\end{array}$ & $\begin{array}{l}.1 \\
.6\end{array}$ \\
\hline OTHER & 0.0 & 0.0 & 0.0 & .3 & .3 & .3 & .3 & .3 & .4 \\
\hline TOTAL FC & 0.0 & 0.0 & 0.0 & 4.1 & 5.3 & 5.3 & 5.4 & 5.5 & 2.4 \\
\hline STARTUP COSTS & 0.0 & 0.0 & .7 & 0.0 & 0.0 & 0.0 & 0.0 & 0.0 & 0.0 \\
\hline TOTAL OPER CDSTS & 0.0 & 0.0 & .7 & 12.6 & 14.5 & 15.2 & 16.2 & 17.2 & 14.9 \\
\hline OPERATING INCOME & 0.0 & 0.0 & -.7 & .3 & -.6 & -.2 & .1 & .4 & 4.0 \\
\hline INTEREST & 0.0 & $\bullet .0$ & 0.0 & 2.1 & 2.0 & 1.9 & 1.8 & 1.7 & 1.5 \\
\hline INC. BEF. LCF/TAX & 0.0 & 0.0 & -.7 & -1.9 & -2.7 & -2.1 & -1.8 & -1.3 & 2.5 \\
\hline LCF USED & 0.0 & 0.0 & 0.0 & 0.0 & 0.0 & 0.0 & 0.0 & 0.0 & 2.5 \\
\hline TAXABLE INCOME & 0.0 & 0.0 & -.7 & -1.9 & -2.7 & $-2,1$ & -1.8 & -1.3 & 0.0 \\
\hline $\begin{array}{l}\text { ITC USED } \\
\text { TAXES PAID }\end{array}$ & $\begin{array}{l}0.0 \\
0.0\end{array}$ & $\begin{array}{l}0.0 \\
0.0\end{array}$ & $\begin{array}{l}0.0 \\
0.0\end{array}$ & $\begin{array}{l}0.0 \\
0.0\end{array}$ & $\begin{array}{l}0.0 \\
0.0\end{array}$ & $\begin{array}{l}0.0 \\
0.0\end{array}$ & $\begin{array}{l}0.0 \\
0.0\end{array}$ & $\begin{array}{l}0.0 \\
0.0\end{array}$ & $\begin{array}{l}0.0 \\
0.0\end{array}$ \\
\hline INCOME AFTER TAX & 0.0 & 3.0 & -.7 & -1.9 & -2.7 & -2.1 & -1.8 & -1.3 & 2.5 \\
\hline DEFREC & 0.0 & 0.0 & 0.0 & 2.5 & 3.6 & 3.4 & 3.4 & 3.4 & .1 \\
\hline CHG IN OTH ASSETS & 0.0 & 3.0 & -1.2 & 0.0 & 0.0 & 0.0 & 0.0 & 0.0 & 0.0 \\
\hline PRINC REPAY & 0.0 & 0.0 & 0.0 & -.6 & -.7 & -.8 & -.9 & $-1,1$ & -1.2 \\
\hline CHG IN WC & 0.0 & 0.0 & -1.9 & -.1 &,- 1 & -.1 & -.1 &.- .1 & -.1 \\
\hline CHG IN BANK LINE & 0.0 & 2.0 & .9 & .0 & .0 & $\therefore 1$ & $\because 1$ & $\therefore 1$ & .1 \\
\hline ENG $\cos T$ & -.6 & 2.0 & 0.0 & 0.0 & 0.0 & 0.0 & 0.0 & 0.0 & 0.0 \\
\hline CONSTR COST & & -7.4 & -4.6 & 0.0 & 0.0 & 0.0 & 0.0 & 0.0 & 0.0 \\
\hline CONSTR INT & 0.0 & -.7 & -1.9 & 0.0 & 0.0 & 0.0 & 0.0 & 0.0 & 0.0 \\
\hline DEBT FIN & 0.0 & 7.6 & 4.8 & 0.0 & 0.0 & 0.0 & 0.0 & 0.0 & 0.0 \\
\hline $\begin{array}{l}\text { ANNUAL CASH FLOW } \\
\text { CUMUL CASH FLOW }\end{array}$ & $\begin{array}{l}-.6 \\
-.6\end{array}$ & $\begin{array}{l}-2.5 \\
-3.1\end{array}$ & $\begin{array}{l}-4.4 \\
-7.6\end{array}$ & $\begin{array}{r}.0 \\
-7.5\end{array}$ & -.7 .3 & $\begin{array}{r}.5 \\
-6.9\end{array}$ & $\begin{array}{r}.7 \\
-6.2\end{array}$ & $\begin{array}{r}1.0 \\
-5.2\end{array}$ & $\begin{array}{l}1.3 \\
3.9\end{array}$ \\
\hline
\end{tabular}


INCOME AND CAGH FLON (\$ MIL)

\begin{tabular}{|c|c|c|c|c|c|c|c|c|c|}
\hline \multicolumn{10}{|l|}{ REVENUES } \\
\hline $\begin{array}{l}\text { ETHANOL } \\
\text { DDG }\end{array}$ & $\begin{array}{r}14.3 \\
5.7\end{array}$ & $\begin{array}{r}15.4 \\
6.1\end{array}$ & $\begin{array}{r}16.6 \\
6.6\end{array}$ & $\begin{array}{r}18.0 \\
7.1\end{array}$ & $\begin{array}{r}19.4 \\
7.7\end{array}$ & $\begin{array}{r}21.0 \\
8.3\end{array}$ & $\begin{array}{r}22.6 \\
9.0\end{array}$ & $\begin{array}{r}24.4 \\
9.7\end{array}$ & $\begin{array}{l}26.4 \\
10.5\end{array}$ \\
\hline DFY AGENT & .5 & .6 & .6 & .7 & .7 & .8 & .8 & .8 & 1.0 \\
\hline ELECT PWR & 0.0 & 0.0 & 0.0 & 0.0 & 0.0 & 0.0 & 0.0 & 0.0 & 0.0 \\
\hline TOTAL REUS & 20.4 & 22.1 & 23.8 & 25.0 & 27.8 & 30.0 & 32.4 & 35.0 & 37.8 \\
\hline \multicolumn{10}{|l|}{ VARIABLE COST } \\
\hline FEEDSTOCK & 10.7 & 11.5 & 12.4 & 13.4 & 14.5 & 15.6 & 16.9 & 18.3 & 19 \\
\hline WOOD & .8 & .9 & .9 & 1.0 & 1.1 & 1.2 & 1.3 & 1.4 & 1 \\
\hline DRY AGENT & .5 & .6 & .6 & .7 & .7 & .8 & .0 & .9 & \\
\hline ELECT & .6 & .6 & .7 & .7 & .8 & .8 & .9 & 1.0 & 1.1 \\
\hline OTHEF & 1.0 & 1.1 & 1.2 & 1.3 & 1.4 & 1.5 & 1.6 & 1.7 & 1.9 \\
\hline TOTAL UC & 13.5 & 14.6 & 15.8 & 17.1 & 18.4 & 19.9 & 21.5 & 23.2 & 25.1 \\
\hline \multicolumn{10}{|l|}{ FIXED COSTS } \\
\hline LAFOR & 1.5 & 1.6 & 1.7 & 1.9 & 2.0 & 2.2 & 2.4 & 2.6 & \\
\hline DEFREC & $\cdot 1$ & $\cdot 1$ & .1 & .1 & .1 & .1 & .1 & .1 & \\
\hline G $8 A$ & .6 & .6 & .7 & .8 & .8 & .9 & 1.0 & 1.0 & 1.12 \\
\hline OTHER & .4 & .4 & .5 & $\therefore 5$ & .5 & .6 & .6 & .7 & \\
\hline TOTAL FC & 2.6 & 2.8 & 3.0 & 3.2 & 3.5 & 3.7 & 4.0 & 4.4 & 4.7 \\
\hline STARTUP COSTS & 0.0 & 0.0 & 0.0 & 0.0 & 0.0 & 0.0 & 0.0 & 0.0 & 0.0 \\
\hline TOTAL OPER COSTS & 16.1 & 17.4 & 18.8 & 20.3 & 21.9 & 23.6 & 25.5 & 27.6 & 29.8 \\
\hline OPERATING INCOME & 4.3 & 4.7 & 5.1 & 5.5 & 5.9 & 6.4 & 6.9 & 7.5 & 8.1 \\
\hline INTEREST & 1.3 & 1.1 & .9 & .6 & .3 & .3 & .3 & .3 & .3 \\
\hline INC, BEF, LCF/TAX & 3.0 & 3.5 & 4.2 & 4.9 & 5.7 & 6.1 & 6.6 & 7.2 & 7.8 \\
\hline LCF USED & 3.0 & 3.5 & 1.5 & 0.0 & 0.0 & 0.0 & 0.0 & 0.0 & 0.0 \\
\hline TAXABLE INCOME & 0.0 & 0.0 & 2.7 & 4.9 & 5.7 & 6.1 & 6.6 & 7.2 & 7.8 \\
\hline $\begin{array}{l}\text { ITC. USED } \\
\text { TAXES PAID }\end{array}$ & $\begin{array}{l}0.0 \\
0.0\end{array}$ & $\begin{array}{l}0.0 \\
0.0\end{array}$ & $\begin{array}{l}1.4 \\
0.0\end{array}$ & $\begin{array}{r}1.8 \\
.6\end{array}$ & $\begin{array}{l}0.0 \\
2.8\end{array}$ & $\begin{array}{l}0.0 \\
3.1\end{array}$ & $\begin{array}{l}0.0 \\
3.3\end{array}$ & $\begin{array}{l}0.0 \\
3.6\end{array}$ & $\begin{array}{l}0.0 \\
3.9\end{array}$ \\
\hline INCOME AFIER TAX & 3.0 & 3.5 & 4.2 & 4.2 & 2.8 & 3.1 & 3.3 & 3.6 & 3.9 \\
\hline DEFREC & .1 & .1 & .1 & .1 & .1 & .1 & .1 & .1 & \\
\hline CHG IN OTH ASSETS & 0.0 & 0.0 & 0.0 & 0.0 & 0.0 & 0.0 & 0.0 & 0.0 & 0.0 \\
\hline $\begin{array}{l}\text { PRINC REFAY } \\
\text { CHG IN WC }\end{array}$ & -1.4 & -1.6 & -1.9 & -2.2 & 0.0 & 0.0 & 0.0 & 0.0 & $\begin{array}{l}0.0 \\
-.3\end{array}$ \\
\hline $\begin{array}{l}\text { CHG IN WC } \\
\text { CHG IN BANK LINE }\end{array}$ & -.1 & -.2 & -.2 & -.2 & -.2 & -.2 & -.2 & -.2 & $\begin{array}{r}-.3 \\
.1\end{array}$ \\
\hline ENG COST & 0.0 & 0.0 & 0.0 & 0.1 & $\therefore 1$ & $\therefore 1$ & $\therefore 1$ & $\therefore 1$ & 0.0 \\
\hline CONSTR COST & 0.0 & 0.0 & 0.0 & 0.0 & $\begin{array}{l}0.0 \\
0.0\end{array}$ & $\begin{array}{l}0.0 \\
0.0\end{array}$ & $\begin{array}{l}0.0 \\
0.0\end{array}$ & $\begin{array}{l}0.0 \\
0.0\end{array}$ & 0.0 \\
\hline CONSTR INT & 0.0 & 0.0 & 0.0 & 0.0 & 0.0 & 0.0 & 0.0 & 0.0 & 0.0 \\
\hline DEBT FIN & 0.0 & 0.0 & 0.0 & 0.0 & 0.0 & 0.0 & 0.0 & 0.0 & 0.0 \\
\hline ANNUAL CASH FLOW & 1.6 & 1.9 & 2.3 & 2.0 & -2.8 & 3.0 & 3.3 & 3.5 & 3.8 \\
\hline & -2.4 & $\cdots .5$ & 1.8 & 3.8 & 6.6 & 9.7 & 12.9 & 16.5 & 20.3 \\
\hline
\end{tabular}


ASSUMFTIONS (1982 \$)

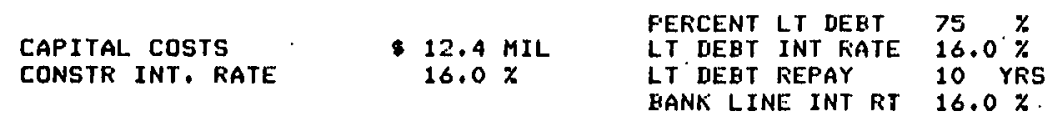

ETH SALE PRICE 1.50 $5 / \mathrm{GAL}$

AET ETH PRICE .70 \$ / GAL

IDG PRICE 170.00 \%TOW

CORN COST : $2.30 \$ / \mathrm{BU}$

$\begin{array}{ll}\text { ELECTR SALES FRICE } & 5.0 \mathrm{C} / \mathrm{KWH} \\ \text { ELECTR FURCH COST } & 5.0 \mathrm{C} / \mathrm{KWH}\end{array}$

INFLATION RATE

INFLATION RATE
CAP COST REAL ESC

$0.0 x$
$5.0 \%$

IAYS REC ETH/DRY AG

IAYS REC DDG

30
30
30

ETH REAL ESC

CORN 8 DDG REAL ESC $0.0 \%$

ELECTR REAL ESC $0.0 \%$

WOOD REAL ESC $0.0 \%$

TAYS REC ELECTR

DAYS FAY CORN

DAYS F'AY WOOD

LIFO INUENTORY POLICY

15 DAYS ETH STORAGE

15 DAYS CORN STORAGE

15 DAYS DDG STORAGE
15 DAYS WOOD STORAG

$\begin{array}{llll}\text { FED INC TAX } & 46.0 \% & \text { EQUIP IEFR LIFE } & 5 \text { YRS } \\ \text { STATE INC TAX } & 4.0 \% & \text { EQLIP ITC } & 20 \% \\ \text { STATE SALES TAX EXEAPT } & 0.0 \% \text { C/GAL } & \text { BLDG ITC } & 0 \\ \text { STATE GASOLINE EXCISE TAX EXEMPT } & 4.0 \text { C/GAL } & & \\ \text { FED GASOLINE EXCISE TAX EXEMPT } & 4.0 \text { C/GAL } & & \end{array}$

PESULTS

P.OE $24.0 \times$ NPV (25 $\times$ DISC RATE) $-.3 \mathrm{MIL}$ PAYEACK 1989

MEBT COU: $\quad 1.5$ IN OPER YR $1 \quad 2.1$ IN OPER YR 6

ENG COSTS \$.6 MIL INITIAL DEBT $\$ 12.4 \mathrm{MIL}$

MAXIMUM CASH EQUITY $\$ 7.5 \mathrm{MIL}$

ANNUAL SALES: $\quad 4.8 \mathrm{MIL}$ GALS ETH 16649 TONS DNG

$0.0 \mathrm{MW} \mathrm{ELECT}$ 
INCOME AND CASH FLOW (\$ MIL)

\begin{tabular}{|c|c|c|c|c|c|c|c|c|c|}
\hline REUENUES & 1982 & 1903 & 1984 & 1985 & 1986 & 1987 & 1988 & 1989 & 1990 \\
\hline $\begin{array}{l}\text { ETHANOL } \\
\text { IIRG }\end{array}$ & 0.0 & 0.0 & 0.0 & 9.0 & 9.7 & 10.5 & 11.3 & 12.2 & 13.2 \\
\hline & 0.0 & 0.0 & 0.0 & 3.6 & 3.9 & 4.2 & 4.5 & 4.9 & 5.2 \\
\hline DRY AGENT & 0.0 & 0.0 & 0.0 & .3 & .4 & .4 & .4 & .4 & .5 \\
\hline ELECT FWR & 0.0 & 0.0 & 0.0 & 0.0 & 0.0 & 0.0 & 0.0 & 0.0 & 0.0 \\
\hline TOTAL REUS & 0.0 & 0.0 & 0.0 & 12.9 & 13.9 & 15.0 & 16.2 & 17.5 & 18.9 \\
\hline VARIABLE COST & & & & & & & & & \\
\hline FEEIISTOCK & 0.0 & 0.0 & 0.0 & 5.5 & 6.0 & 6.4 & 6.9 & 7.5 & 8.1 \\
\hline wood & 0.0 & 0.0 & .0 .0 & .5 & .5 & .6 & .6 & .7 & .7 \\
\hline DRY AGENT & 0.0 & $0 . c$ & 0.0 & .3 & .4 & .4 & $\therefore 4$ & .4 & .5 \\
\hline ELECT & 0.0 & 0.0 & 0.0 & .4 & .4 & .4 & .5 & .5 & .5 \\
\hline OTHEK & 0.0 & 0.0 & 0.0 & .6 & .7 & .7 & .8 & .9 & .9 \\
\hline TOTAL VC & 0.0 & 0.0. & 0.0 & 7.3 & 7.9 & 8.6 & 9.2 & 10.0 & 10.8 \\
\hline FIXED COSTS & & & & & & & & & \\
\hline LABOR & 0.0 & 0.0 & 0.0 & .9 & 1.0 & 1.1 & 1.2 & 1.3 & .1 .4 \\
\hline $\begin{array}{l}\text { DEPREC } \\
\text { G \& A }\end{array}$ & 0.0 & 0.0 & 0.0 & 2.5 & 3.6 & 3.4 & 3.4 & 3,4 & .1 \\
\hline $\begin{array}{l}\text { G \& A A } \\
\text { OTHER }\end{array}$ & $\begin{array}{l}0.0 \\
0.0\end{array}$ & $\begin{array}{l}0.0 \\
0.0\end{array}$ & 0.0 & .3 & .4 & & .5 & .5 & $\begin{array}{r}.6 \\
.4\end{array}$ \\
\hline TOTAL FC & 0.0 & 0.0 & 0.0 & 4.1 & 5.3 & 5.3 & 5.4 & 5.5 & 2.4 \\
\hline STARTUP COSTS & 0.0 & 0.0 & .7 & 0.0 & 0.0 & 0.0 & 0.0 & 0.0 & 0.0 \\
\hline TOTAL OPER COSTS & 0.0 & 0.0 & .7 & 11.4 & 13.2 & 13.8 & 14.6 & 15.5 & 13.2 \\
\hline OPERATING INCOME & 0.0 & 0.0 & -.7 & 1.5 & .7 & 1.2 & 1.6 & 2.0 & 5.7 \\
\hline INTEREST & 0.0 & 0.3 & 0.0 & 2.1 & 2.0 & 1.9 & 1.8 & 1.7 & 1.5 \\
\hline INC, BEF, LCF/TAX & 0.0 & 0.2 & -.7 & -.7 & -1.4 & -.7 & -.2 & .3 & 4.2 \\
\hline LCF USED & 0.0 & 0.0 & 0.0 & 0.0 & 0.0 & 0.0 & 0.0 & .3 & 3.4 \\
\hline TAXABLE INCOME & 0.0 & 0.0 & -.7 & -.7 & -1.4 & -.7 & -.2 & 0.0 & .8 \\
\hline $\begin{array}{l}\text { ITC USED } \\
\text { TAXES PAID }\end{array}$ & $\begin{array}{l}0.0 \\
0.0\end{array}$ & $\begin{array}{l}0.0 \\
0.0\end{array}$ & $\begin{array}{l}0.0 \\
0.0\end{array}$ & $\begin{array}{l}0.0 \\
0.0\end{array}$ & $\begin{array}{l}0.0^{\circ} \\
0.0\end{array}$ & $\begin{array}{l}0.0 \\
0.0\end{array}$ & $\begin{array}{l}0.0 \\
0.0\end{array}$ & $\begin{array}{l}0.0 \\
0.0\end{array}$ & $\begin{array}{r}.4 \\
0.0\end{array}$ \\
\hline INCOME AFTER TAX & 0.0 & 0.0 & -.7 & -.7 & -1.4 & -.7 & -.2 & .3 & 4.2 \\
\hline DEPREC & 0.0 & 0.0 & 0.0 & 2.5 & 3.6 & 3.4 & 3.4 & 3.4 & .1 \\
\hline CHG IN OTH ASSETS & 0.0 & 0.0 & -1.2 & 0.0 & 0.0 & 0.0 & 0.0 & 0.0 & 0.0 \\
\hline PRINC REPAT & 0.0 & 0.0 & 0.0 & -.6 & -.7 & -.8 & -.9 & -1.1 & -1.2 \\
\hline CHG IN WC & 0.0 & 0.0 & -1.8 & -.1 & -.1 & -.1 & -.1 & -.1 & -.1 \\
\hline CHG IN BANK LINE & 0.0 & 0.0 & .9 & .0 & .0 & .1 & .1 & .1 & .1 \\
\hline ENG COST & -.6 & 0.0 & 0.0 & 0.0 & 0.0 & 0.0 & 0.0 & 0.0 & 0.0 \\
\hline CONSTR COST & 0.0 & -9.4 & -4.6 & 0.0 & 0.0 & 0.0 & 0.0 & 0.0 & 0.0 \\
\hline CONSTR INT & 0.0 & -.7 & -1.9 & 0.0 & 0.0 & 0.0 & 0.0 & 0.0 & 0.0 \\
\hline DEBT FIN & 0.0 & 7.6 & 4.8 & 0.0 & 0.0 & 0.0 & 0.0 & 0.0 & 0.0 \\
\hline ANNUAL CASH FLOW & -.6 & $-2,5$ & -4.4 & 1.2 & 1.5 & $\begin{array}{r}1.9 \\
-2.9\end{array}$ & 2.2 & 2.6 & 3.0 \\
\hline & & -3.1 & $-7 \cdot 5$ & -6.3 & -4.8 & -2.9 & -.7 & 1.9 & 4.9 \\
\hline
\end{tabular}




\begin{tabular}{|c|c|c|c|c|c|c|c|c|c|}
\hline REUENUES & 1991 & 1902 & 1993 & 1994 & 1995 & 1996 & 1997 & 1998 & 1999 \\
\hline $\begin{array}{l}\text { ETHANOL } \\
\text { DDG } \\
\text { DRY AGENT } \\
\text { ELECT FWR }\end{array}$ & $\begin{array}{r}14.3 \\
5.7 \\
.5 \\
0.0\end{array}$ & $\begin{array}{r}15.4 \\
6.1 \\
.6 \\
0.0\end{array}$ & $\begin{array}{r}16.6 \\
6.6 \\
0.6 \\
0.0\end{array}$ & $\begin{array}{r}18.0 \\
7.1 \\
.7 \\
0.0\end{array}$ & $\begin{array}{r}19.4 \\
7.7 \\
0.7\end{array}$ & $\begin{array}{r}21.0 \\
8.3 \\
.8 \\
0.0\end{array}$ & $\begin{array}{r}22.6 \\
9.0 \\
.8 \\
0.0\end{array}$ & $\begin{array}{r}24.4 \\
9.7 \\
0.9 \\
0.0\end{array}$ & $\begin{array}{r}26.4 \\
10.5 \\
1.0 \\
0.0\end{array}$ \\
\hline TOTAL REVS & 20.4 & 22.1 & 23.8 & 25.8 & 27.8 & 30.0 & 32.4 & 35.0 & 37.8 \\
\hline $\begin{array}{l}\text { VARIABLE COST } \\
\text { FEEDSTOCK } \\
\text { WOOD } \\
\text { DRY AGENT } \\
\text { ELECT } \\
\text { OTHER }\end{array}$ & $\begin{array}{r}8.7 \\
.8 \\
.5 \\
.6 \\
1.0\end{array}$ & $\begin{array}{r}9.4 \\
.9 \\
.6 \\
.6 \\
1.1\end{array}$ & $\begin{array}{r}10.2 \\
.9 \\
.6 \\
.7 \\
1.2\end{array}$ & $\begin{array}{r}: \\
11.0 \\
1.0 \\
: 7 \\
: 7 \\
1.3\end{array}$ & $\begin{array}{r}11.9 \\
1.1 \\
.7 \\
.8 \\
1.4\end{array}$ & $\begin{array}{r}12.9 \\
1.2 \\
.8 \\
.8 \\
1.5\end{array}$ & $\begin{array}{r}13.9 \\
1.3 \\
.8 \\
.9 \\
1.6\end{array}$ & $\begin{array}{r}15.0 \\
1.4 \\
.9 \\
1.0 \\
1.7\end{array}$ & $\begin{array}{r}16.2 \\
1.5 \\
1.0 \\
1.1 \\
1.9\end{array}$ \\
\hline TOTAL VC & 11.6 & 12.6 & 13.6 & 14.7 & 15.8 & 17.1 & 18.5 & 20.0 & 21.5 \\
\hline $\begin{array}{c}\text { FIXED COSTS } \\
\text { LABOR } \\
\text { DEFREC } \\
\text { G \& A A } \\
\text { OTHER }\end{array}$ & $\begin{array}{r}1.5 \\
.1 \\
.6 \\
.4\end{array}$ & $\begin{array}{l}1.6 \\
.1 \\
.6 \\
.4\end{array}$ & $\begin{array}{r}1.7 \\
.1 \\
.7 \\
.5\end{array}$ & $\begin{array}{r}1.9 \\
.1 \\
.8 \\
.5\end{array}$ & $\begin{array}{r}2.0 \\
.1 \\
.8 \\
.5\end{array}$ & $\begin{array}{r}2.2 \\
.1 \\
.9 \\
.6\end{array}$ & $\begin{array}{l}2.4 \\
.1 \\
1.0 \\
.6\end{array}$ & $\begin{array}{l}2.6 \\
.1 \\
1.0 \\
.7\end{array}$ & $\begin{array}{l}2.8 \\
.1 \\
1.1 \\
.7\end{array}$ \\
\hline TOTAL FC & 2.6 & 2.8 & 3.0 & 3.2 & 3.5 & 3.7 & 4.0 & 4.4 & 4.7 \\
\hline STARTUP COSTS & 0.0 & 0.0 & 0.0 & 0.0 & 0.0 & 0.0 & 0.0 & 0.0 & 0.0 \\
\hline TOTAL OPER COSTS & 14.2 & $1 E .3$ & 16.6 & 17.9 & 19.3 & 20.8 & 22.5 & 24.3 & 26.2 \\
\hline OPERATING INCOME & 6.2 & 6.7 & 7.3 & 7.9 & 8.5 & 9.2 & 9.9 & 10.7 & 11.6 \\
\hline INTEREST & 1.3 & 1.1 & .9 & .66 & .2 & .3 & .3 & .3 & .3 \\
\hline INC. BEF. LCF/TAX & 4.9 & 5.6 & 6.4 & 7.3 & £.3 & 8.9 & 9.7 & 10.4 & 11.3 \\
\hline LCF USED & 0.0 & 0.0 & 0.0 & 0.0 & 0.0 & 0.0 & 0.0 & 0.0 & 0.0 \\
\hline TAXABLE INCOME & 4.9 & 5.6 & 6.4 & 7.3 & 61.3 & 8.9 & 9.7 & 10.4 & 11.3 \\
\hline $\begin{array}{l}\text { ITC USED } \\
\text { TAXES PAII }\end{array}$ & $\begin{array}{l}2.4 \\
0.0\end{array}$ & $\begin{array}{l}.3 \\
2.5\end{array}$ & $\begin{array}{l}0.0 \\
3.2\end{array}$ & $\begin{array}{l}0.0 \\
3.6\end{array}$ & $\begin{array}{l}0.0 \\
4.1\end{array}$ & $\begin{array}{l}0.0 \\
4.5\end{array}$ & $\begin{array}{l}0.0 \\
4.8\end{array}$ & $\begin{array}{l}0.0 \\
5.2\end{array}$ & $\begin{array}{l}0.0 \\
5.6\end{array}$ \\
\hline INCOME AFTER TAX & 4.9 & 3.1 & 3.2 & 3.6 & 4.1 & 4.5 & 4.8 & 5.2 & 5.6 \\
\hline $\begin{array}{l}\text { DEPREC } \\
\text { CHE IN OTH ASSETS } \\
\text { PRINC REPAY } \\
\text { CHG IN WC } \\
\text { CHE IN BANK LINE } \\
\text { ENG COST } \\
\text { CONSTR COST } \\
\text { CONSTR INT } \\
\text { IIEBT FIN }\end{array}$ & $\begin{array}{r}.1 \\
0.0 \\
-1.4 \\
-.1 \\
.1 \\
0.0 \\
0.0 \\
0.0 \\
0.0\end{array}$ & $\begin{aligned} .1 \\
0.0 \\
-1.0 \\
-.02 \\
.1 \\
2.0 \\
0.0 \\
0.0 \\
0.0\end{aligned}$ & $\begin{array}{r}.1 \\
0.0 \\
-1.9 \\
-.2 \\
.1 \\
0.0 \\
0.0 \\
0.0 \\
0.0\end{array}$ & $\begin{array}{r}.1 \\
0.0 \\
-2.2 \\
-.2 \\
.1 \\
0.0 \\
0.0 \\
0.0 \\
0.0\end{array}$ & $\begin{array}{l}.1 \\
0.0 \\
0.0 \\
-.2 \\
.1 \\
0.0 \\
0.0 \\
0.0 \\
0.0\end{array}$ & $\begin{array}{l}.1 \\
0.0 \\
0.0 \\
-.2 \\
.1 \\
0.0 \\
0.0 \\
0.0 \\
0.0\end{array}$ & $\begin{array}{l}.1 \\
0.0 \\
0.0 \\
-.2 \\
.01 \\
0.0 \\
0.0 \\
0.0 \\
0.0\end{array}$ & $\begin{array}{l}.1 \\
0.0 \\
0.0 \\
-.2 \\
0.1 \\
0.0 \\
0.0 \\
0.0 \\
0.0\end{array}$ & $\begin{array}{l}.1 \\
0.0 \\
0.0 \\
-.3 \\
.1 \\
0.0 \\
0.0 \\
0.0 \\
0.0\end{array}$ \\
\hline $\begin{array}{l}\text { ANNUAL CASH FLOW } \\
\text { CUMUL CASH FLOW }\end{array}$ & $\begin{array}{l}3.5 \\
8.4\end{array}$ & $\begin{array}{l}1.5 \\
9.8\end{array}$ & $\begin{array}{r}1.3 \\
11.1\end{array}$ & $\begin{array}{r}1.4 \\
12.5\end{array}$ & $\begin{array}{r}4.1 \\
16.6\end{array}$ & $\begin{array}{r}4.4 \\
21.1\end{array}$ & $\begin{array}{r}4.8 \\
25.9\end{array}$ & $\begin{array}{r}5.2 \\
31.0\end{array}$ & $\begin{array}{r}5.6 \\
36.6\end{array}$ \\
\hline
\end{tabular}


ASSUMFTIONS (1982 \$)

14285 GFD RATEU CAP
$2.5 \mathrm{GAL} \mathrm{ETH} / \mathrm{BL}$

18 LBS DDG/BU
CAFITAL COSTS

CONSTR INT. RATE

$\begin{array}{lll}\$ 12.4 \mathrm{MIL} & \text { PERCENT LT UEET } & 75 \% \\ 16.0 \% & \text { LT DEBT INT RATE } & 16.0 \% \\ & \text { LT IEET REFAY } & 10 \% \text { YRS } \\ & \text { BANK LINE INT RT } & 16.0 \%\end{array}$

ETH SALE PRICE $1.30 \$ / \mathrm{GAL}$

NET ETH FRICE .50 \%AL

DDG PRICE $170.00 \quad 1 / T O N$

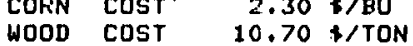

DAYS REC ETH/DRY AG

DAYS REC DDG

30

DAYS REC ELECTR

\section{ELECTR SALES PRICE} ELECTR PUFCH COST

\section{$5.0 \mathrm{C} / \mathrm{KWH}$ $5.0 \mathrm{C} / \mathrm{KWH}$}

DAYS FAY COFN DAYS FAY WOOD
INFLATION RATE CAP COST REAL ESC

ETH REAL ESC

\& $0.0 x$

ELECTR REAL ESC

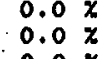

$\begin{array}{lclr}\text { FED INC TAX } & 46.0 \% & \text { EQUIP IEFR LIFE } & 5 \% \text { YRS } \\ \text { STATE INC TAX } & 4.0 \% & \text { EDUIP ITC } & 20 \% \\ \text { STATE SALES TAX EXEMFT } & 0.0 \text { C/GAL } & \text { BLDG ITC } & 0 \% \\ \text { STATE GASOLINE EXCISE TAX EXEMFT } & 4.0 \text { C/GAL } & & \\ \text { FED GASOLINE EXCISE TAX EXEMFT } & 4.0 \text { C/GAL } & & \end{array}$

RESULT

ROE $13.4 \times$ NFU (25 \% DISC RATE) -3.2 MIL PAYBACK 1993

DEBT COV:

1.0 IN OPER YR

1.5 IN OPER YR 6

ENG COSTS \$ .6 MIL

INITIAL DEBT $\$ 12.4 \mathrm{MIL}$

MAXIMUM CASH EQUiTY \$7.5 MIL

ANNUAL SALES:

4.8 MIL GALS ETH

16649 TONS ING

$0.0 \mathrm{MW}$ ELECT

ANNUAL FLANT FEQS AT FULL CAFACITY

$$
\begin{aligned}
& 1.9 \text { MIL EU CORN } \\
& 6 \text { MIL KWH }
\end{aligned}
$$


INCOME AND CAGH FLOW (\$ MIL)

\begin{tabular}{|c|c|c|c|c|c|c|c|c|c|}
\hline REUENUES & 1982 & 1983 & 1994 & 1905 & 1986 & 1987 & 1988 & 1989 & 1990 \\
\hline $\begin{array}{l}\text { ETHANOL } \\
\text { DIG } \\
\text { DRY AGENT } \\
\text { ELECT FWR }\end{array}$ & $\begin{array}{l}0.0 \\
0.0 \\
0.0 \\
0.0\end{array}$ & $\begin{array}{l}0.0 \\
0.0 \\
0.0 \\
0.0\end{array}$ & $\begin{array}{l}0.0 \\
0.0 \\
0.0 \\
0.0\end{array}$ & $\begin{array}{l}7.8 \\
3.6 \\
.3 \\
0.0\end{array}$ & $\begin{array}{l}8.4 \\
3.9 \\
.4 \\
0.0\end{array}$ & $\begin{array}{l}9.1 \\
4.2 \\
.4 \\
0.0\end{array}$ & $\begin{array}{r}9.8 \\
4.5 \\
.4 \\
0.0\end{array}$ & $\begin{array}{r}10.6 \\
4.9 \\
.4 \\
0.0\end{array}$ & $\begin{array}{r}11.4 \\
5.2 \\
.5 \\
0.0\end{array}$ \\
\hline TOTAL REUSS & 0.0 & 0.0 & 0.0 & 11.7 & 12.6 & 13.6 & 14.7 & 15.9 & 17.2 \\
\hline $\begin{array}{l}\text { VARIABLE COST } \\
\text { FEEDSTOCK } \\
\text { WOOD } \\
\text { DRY AGENT } \\
\text { ELECT } \\
\text { OTHER }\end{array}$ & $\begin{array}{l}0.0 \\
0.0 \\
0.0 \\
0.0 \\
0.0\end{array}$ & $\begin{array}{l}0.0 \\
0.0 \\
0.0 \\
0.0 \\
0.0\end{array}$ & $\begin{array}{l}0.0 \\
0.0 \\
0.0 \\
0.0 \\
0.0\end{array}$ & $\begin{array}{r}5.5 \\
.5 \\
.3 \\
.4 \\
.6\end{array}$ & $\begin{array}{r}6.0 \\
.5 \\
.4 \\
.4 \\
.7\end{array}$ & $\begin{array}{r}6.4 \\
.6 \\
.4 \\
.4 \\
.7\end{array}$ & $\begin{array}{r}6.9 \\
.6 \\
.4 \\
.5 \\
. \quad .8\end{array}$ & $\begin{array}{l}7.5 \\
.7 \\
.4 \\
.5\end{array}$ & $\begin{array}{r}8.1 \\
.7 \\
.5 \\
.5 \\
.9\end{array}$ \\
\hline TOTAL VC & 0.0 & 0.0 & 0.0 & 7.3 & 7.9 & 8.6 & 9.2 & 10.0 & 10.8 \\
\hline $\begin{array}{l}\text { FIXED COSTS } \\
\text { LABOR } \\
\text { DEPREC } \\
\text { G\& \& } \\
\text { OTHER }\end{array}$ & $\begin{array}{l}0.0 \\
0.0 \\
0.0 \\
0.0\end{array}$ & $\begin{array}{l}0.0 \\
0.0 \\
0.0 \\
0.0\end{array}$ & $\begin{array}{l}0.0 \\
0.0 \\
0.0 \\
0.0\end{array}$ & $\begin{array}{r}.9 \\
2.5 \\
.4 \\
.3\end{array}$ & $\begin{array}{r}1.0 \\
3.6 \\
.4 \\
.3\end{array}$ & $\begin{array}{r}1.1 \\
3.4 \\
.4 \\
.3\end{array}$ & $\begin{array}{r}1.2 \\
3.4 \\
.5 \\
.3\end{array}$ & $\begin{array}{r}1.3 \\
3.4 \\
.5 \\
.3\end{array}$ & $\begin{array}{r}1.4 \\
.1 \\
.6 \\
.4\end{array}$ \\
\hline TOTAL FC & 0.0 & 0.0 & 0.0 & 4.1 & 5.3 & 5.3 & 5.4 & 5.5 & 2,4 \\
\hline STARTUP COSTS & 0.0 & 0.0 & .7 & 0.0 & 0.0 & 0.0 & 0.0 & 0.0 & 0.0 \\
\hline TOTAL OPER COSTS: & 0.0 & 0.0 & .7 & 11.4 & 13.2 & 13.8 & 14.6 & 15.5 & 13.2 \\
\hline OPERATING INCOME & 0.0 & 0.0 & -.7 & .3 & -.6 & -.2 & .1 & .4 & 4.0 \\
\hline INTEREST & 0.0 & 0.0 & 0.0 & 2.1 & 2.0 & 1.9 & 1.8 & 1.7 & 1.5 \\
\hline INC. BEF. LCF/TAX & 0.0 & 0.0 & -.7 & -1.9 & -2.6 & -2.1 & -1.7 & -1.3 & 2.5 \\
\hline LCF USED & 0.0 & 0.0 & 0.0 & 0.0 & 0.0 & 0.0 & 0.0 & 0.0 & 2.5 \\
\hline TAXABLE INCOME & 0.0 & 0.0 & -.7 & -1.9 & -2.6 & -2.1 & -1.7 & -1.3 & 0.0 \\
\hline $\begin{array}{l}\text { ITC USED } \\
\text { TAXES PAID }\end{array}$ & $\begin{array}{l}0.0 \\
0.0\end{array}$ & $\begin{array}{l}0.0 \\
0.0\end{array}$ & $\begin{array}{l}0.0 \\
0.0\end{array}$ & $\begin{array}{l}0.0 \\
0.0\end{array}$ & $\begin{array}{l}0.0 \\
0.0\end{array}$ & $\begin{array}{l}0.0 \\
0.0\end{array}$ & $\begin{array}{l}0.0 \\
0.0\end{array}$ & $\begin{array}{l}0.0 \\
0.0\end{array}$ & $\begin{array}{l}0.0 \\
0.0\end{array}$ \\
\hline INCOME AFTER TAX & 0.0 & 0.0 & -.7 & -1.9 & -2.6 & -2.1 & -1.7 & -1.3 & 2.5 \\
\hline $\begin{array}{l}\text { DEFREC } \\
\text { CHG IN OTH ASSETS } \\
\text { PRINC REPAY } \\
\text { CHG IN WC } \\
\text { CHG IN BANK LINE } \\
\text { ENG COST } \\
\text { CONSTR COST } \\
\text { CONSTR INT } \\
\text { DEBT FIN }\end{array}$ & $\begin{array}{l}0.0 \\
0.0 \\
0.0 \\
0.0 \\
0.0 \\
-.0 \\
0.0 \\
0.0 \\
0.0\end{array}$ & $\begin{array}{r}0.0 \\
0.0 \\
0.0 \\
0.0 \\
0.0 \\
0.0 \\
-9.4 \\
-.7 \\
7.6\end{array}$ & $\begin{array}{r}0.0 \\
-1.2 \\
0.0 \\
-1.7 \\
.8 \\
0.0 \\
-4.6 \\
-1.9 \\
4.8\end{array}$ & $\begin{array}{l}2.5 \\
0.0 \\
-.6 \\
-.1 \\
.0 \\
0.0 \\
0.0 \\
0.0 \\
0.0\end{array}$ & $\begin{array}{l}3.6 \\
0.0 \\
-.7 \\
-.1 \\
.0 \\
0.0 \\
0.0 \\
0.0 \\
0.0\end{array}$ & $\begin{array}{l}3.4 \\
0.0 \\
-.8 \\
-.1 \\
.0 \\
0.0 \\
0.0 \\
0.0 \\
0.0\end{array}$ & $\begin{array}{l}3.4 \\
0.0 \\
-.9 \\
-.1 \\
.1 \\
0.0 \\
0.0 \\
0.0 \\
0.0\end{array}$ & $\begin{array}{r}3.4 \\
0.0 \\
-1.1 \\
-.1 \\
.1 \\
0.0 \\
0.0 \\
0.0 \\
0.0\end{array}$ & $\begin{array}{r}. .1 \\
0.0 \\
-1.2 \\
-.1 \\
.1 \\
0.0 \\
0.0 \\
0.0 \\
0.0\end{array}$ \\
\hline $\begin{array}{l}\text { ANNUAL CASH FLOW } \\
\text { CUMUL CASH FLOU }\end{array}$ & $\begin{array}{l}-.6 \\
-.6\end{array}$ & $\begin{array}{l}-2.5 \\
-3.1\end{array}$ & $\begin{array}{l}-4.3 \\
-7.5\end{array}$ & $\begin{array}{r}.0 \\
-7.4\end{array}$ & -7.2 & -6.5 & $\begin{array}{r}.7 \\
-.6 .0\end{array}$ & $\begin{array}{r}1.0 \\
-5.0\end{array}$ & $\begin{array}{r}1.3 \\
-3.7\end{array}$ \\
\hline
\end{tabular}


INCOME AND CASH FLOW (\$ MIL)

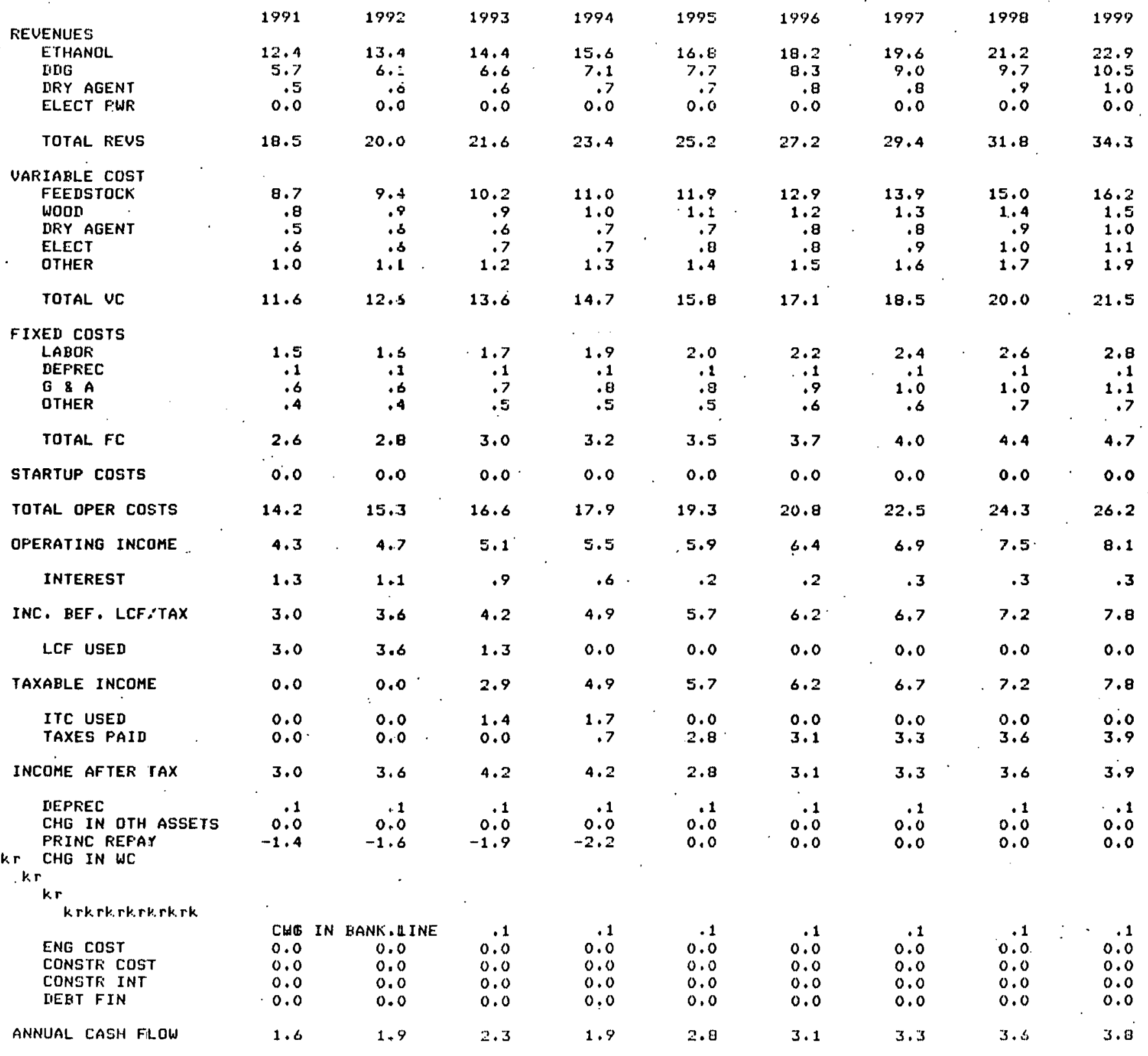


CORN - TO-ETHANOL FLANT

$4.8 \mathrm{MIL}$ GFY RATEN CAFACITY

ASSUMFTIONS (1902\$)

14285 GFD RATED CAF

CAPITAL COSTS
CONSTR INT. FATE

$\begin{array}{lll}12.4 \mathrm{MIL} & \text { PERCENT LT DEBT } & 75 \% \\ 16.0 \% & \text { LT DEBT INT.FATE } & 16.0 \% \\ & \text { LT DEBT REFAY } & 10 \% \text { YRS }\end{array}$

INFLATION RATE

CAF COST REAL

$8.0 \%$ $\begin{array}{ll}\text { LT DEBT REPAY } & 10 \\ \text { BANK LINE INT. RT } & 16.0 \mathrm{X}\end{array}$

ETH SALE PRICE $1.70 \$ / \mathrm{GAL}$ NET ETH FRICE .90 \$/GAL DDG PFICE $170.00 \$ / T O N$ CORN COST $2.80 \$ 1 \mathrm{DU}$

\begin{tabular}{|c|c|c|c|}
\hline $\begin{array}{l}\text { ELECTR SALES PRICE } \\
\text { ELECTR PURCH COST }\end{array}$ & $\begin{array}{l}5.0 \mathrm{C} / \mathrm{KWH} \\
5.0 \mathrm{C} / \mathrm{KWH}\end{array}$ & $\begin{array}{l}\text { ETH REAL ESC } \\
\text { CORN \& DDG REAL. ESC. } \\
\text { ELECTR REAL ESC } \\
\text { WOODD REAL ESC }\end{array}$ & $\begin{array}{l}0.0 \% \\
0.0 \% \\
.0 .0 \% \\
0.0 \%\end{array}$ \\
\hline
\end{tabular}

DAYS REC ETH/DRY AG 30 $\begin{array}{ll}\text { DAYS REC DDG } & 30 \\ \text { DAYS REC ELECTR } & \mathbf{3 0}\end{array}$ DAYS PAY CORN
DAYS FAY WOOD
LIFO INUENTORY FOLICY

15 DAYS ETH STORAGE

15 DAYS CORN STORAGE

15 DAYS DUG STORAGE

\section{FED INC TAX}

STATE INC TAX

STATE SALES TAX EXEMPT

STATE GASOLINE EXCISE TAX EXEMFT

FED GASOLINE EXCISE TAX EXEMPT

$\begin{array}{rl}46.0 & x \\ 4.0 & x \\ 0.0 & C / G A L \\ 4.0 & C / G A L\end{array}$

EQUIP DEFR LIFE

EQUIP ITC

BLIG ITC

5 YRS

$20 x$
$0 x$

RESULTS

ROE $23.6 \%$ NFU (2S $\%$ DISC RATE) - -.4 MIL FAYBACK 1989

DEBT COV: 1.5 IN OFER YR 1 . 2.1 IN OFER YR 6

ENG COSTS .6 MIL INITIAL DEBT \$12.4 MIL

MAXIMUM CASH EQUITY $\$ 7.6 \mathrm{MIL}$

ANNUAL SALES:

4.8 MIL GALS ETH

16649 TONS DIIG

$0.0 \mathrm{MW} \mathrm{ELCCT}$

ANNUAL FLANT REQS

AT FULL CAFACITY

1.9 MIL EU CORN

29273 ACRES CORNI

13649 HEAL CATTLE 


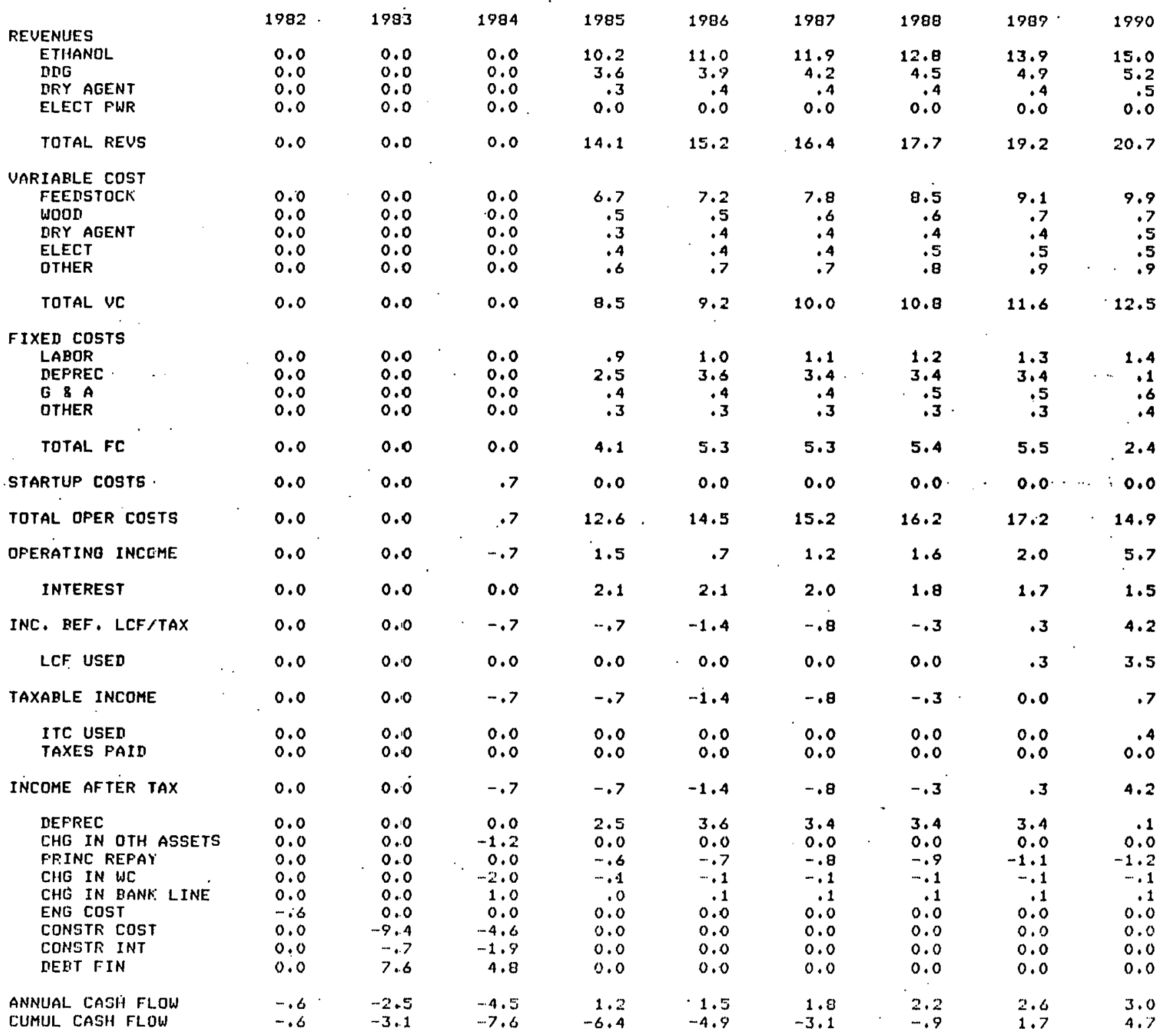


INCOME AND CASH FLOW (\$ MIL)

\begin{tabular}{|c|c|c|c|c|c|c|c|c|c|}
\hline REUENUES & 1991 & 1992 & 1993 & 1994 & 1995 & 1996 & 1997 & 1998 & 1999 \\
\hline ETHANOL & 16.2 & 17.5 & 18.9 & 20.4 & 22.0 & 23.8 & 25.7 & 27.7 & 29.9 \\
\hline DDG & 5.7 & 6.1 & 6.6 & 7.1 & 7.7 & 8.3 & 9.0 & 9.7 & 10.5 \\
\hline DRY AGENT & .5 & .6 & .6 & .7 & .7 & .8 & .8 & .9 & 1.0 \\
\hline ELECT FWR & 0.0 & 0.0 & 0.0 & 0.0 & 0.0 & 0.0 & 0.0 & 0.0 & 0.0 \\
\hline TOTAL REUS & 22.3 & 24.1 & 26.1 & 28.1 & 30.4 & 32.8 & 35.5 & 38.3 & 41.4 \\
\hline VARIARLE COST & & & & & & & & & \\
\hline FEEDSTOCK & 10.7 & 11.5 & 12.4 & 13.4 & 14.5 & 15.6 & 16.9 & 18.3 & 19.7 \\
\hline WOOD & $\cdot 8$ & .9 & .9 & 1.0 & 1.1 & 1.2 & 1.3 & 1.4 & 1.5 \\
\hline DRY AGENT & .5 & .66 & .6 &. .7 & .7 &.$\theta$ & .8 & .9 & 1.0 \\
\hline ELECT & .6 & .6 & .7 & .7 & .8 & .8 & .9 & 1.0 & 1.1 \\
\hline DTHER & 1.0 & 1.1 & 1.2 & 1.3 & 1.4 & 1.5 & 1.6 & 1.7 & 1.9 \\
\hline TOTAL VC & 13.5 & 14.6 & $15: \theta$ & 17.1 & 18.4 & 19.9 & 21.5 & 23.2 & 25.1 \\
\hline FIXED COSTS & & & & & & & & & \\
\hline LABOR & 1.5 & 1.6 & 1.7 & 1.9 & 2.0 & 2.2 & 2.4 & 2.6 & 2.8 \\
\hline DEFREC & .1 & .1 & .1 & .1 & .1 & .1 & .1 & .1 & .1 \\
\hline G \& A & .6 & .6 & .7 & .8 & .8 & .9 & 1.0 & 1.0 & 1.1 \\
\hline OTHER & .4 & .4 & .5 & .5 & .5 & .6 & .6 & .7 & .7 \\
\hline TOTAL FC & 2.6 & $2 \cdot 8$ & 3.0 & 3.2 & 3.5 & 3.7 & 4.0 & 4.4 & 4.7 \\
\hline STARTUP COSTS & 0.0 & 0.0 & 0.0 & 0.0 & 0.0 & 0.0 & 0.0 . & 0.0 & 0.0 \\
\hline TOTAL OPER COSTS & 16.1 & 17.4 & 18.9 & 20.3 & 21.9 & 23.6 & 25.5 & 27.6 & 29.8 \\
\hline OPERATING INCOME & 6.2 & 6.7 & 7.3 & 7.9 & 8.5 & 9.2 & 9.9 & 10.7 & 11.6 \\
\hline INTEREST & 1.4 & 1.1 & .9 & .6 & .3 & .3 & .3 & .3 & .3 \\
\hline INC. BEF. LCF/TAX & 4.9 & 5.6 & 6.4 & 7.3 & 8.2 & 8.9 & 9.6 & 10.4 & 11.3 \\
\hline LCF USED & 0.0 & 0.0 & 0.0 & 0.0 & 0.0 & 0.0 & 0.0 & 0.0 & 0.0 \\
\hline TAXABLE INCOME & 4.9 & 5.6 & 6.4 & 7.3 & 8.2 & 8.9 & 9.6 & 10.4 & 11.3 \\
\hline $\begin{array}{l}\text { ITC USED } \\
\text { TAXES PAID }\end{array}$ & $\begin{array}{l}2.4 \\
0.0\end{array}$ & $\begin{array}{r}.4 \\
2.4\end{array}$ & $\begin{array}{l}0.0 \\
3.2\end{array}$ & $\begin{array}{l}0.0 \\
3.6\end{array}$ & $\begin{array}{l}0.0 \\
4.1\end{array}$ & $\begin{array}{l}0.0 \\
4.5\end{array}$ & $\begin{array}{l}0.0 \\
4.8\end{array}$ & $\begin{array}{l}0.0 \\
5.2\end{array}$ & $\begin{array}{l}0.0 \\
5.6\end{array}$ \\
\hline INCOME AFTER TAX & 4.9 & 3.1 & 3.2 & 3.6 & 4.1 & 4.5 & 4.8 & 5.2 & 5.6 \\
\hline DEPREC & .1 & .1 & 1 & .1 & 1 & .1 & .1 & .1 & .1 \\
\hline CHG IN OTH ASSETS. & 0.0 & 0.0 & 0.0 & 0.0 & 0.0 & 0.0 & 0.0 & 0.0 & 0.0 \\
\hline FRINC REPAY & -1.4 & -1.6 & -1.9 & -2.2 & 0.0 & 0.0 & 0.0 & 0.0 & 0.0 \\
\hline CHG IN WC & -.2 & -.2 & -.2 & -.2 & -.2 & -.2 & -.2 & -.3 & -.3 \\
\hline CHG IN BANK LINE & .1 & .1 & .1 & .1 & .1 & .1 & .1 & $\cdot .1$ & .1 \\
\hline ENG $\cos T$ & 0.0 & 0.0 & 0.0 & 0.0 & 0.0 & 0.0 & 0.0 & 0.0 & 0.0 \\
\hline CONSTR COST & 0.0 & 0.0 & 0.0 & 0.0 & 0.0 & 0.0 & 0.0 & 0.0 & 0.0 \\
\hline CONSTR INT & 0.0 & 0.0 & 0.0 & 0.0 & 0.0 & 0.0 & 0.0 & 0.0 & 0.0 \\
\hline DEET FIN & 0.0 & 0.0 & 0.0 & 0.0 & 0.0 & 0.0 & 0.0 & 0.0 & 0.0 \\
\hline $\begin{array}{l}\text { ANNUAL CASH FLCW } \\
\text { CUMUL CASH FLOL. }\end{array}$ & $\begin{array}{l}3.4 \\
8.1\end{array}$ & $\begin{array}{l}1.5 \\
9.6\end{array}$ & $\begin{array}{r}1.3 \\
10.9\end{array}$ & $\begin{array}{r}1.4 \\
12.3\end{array}$ & $\begin{array}{r}4.1 \\
16.4\end{array}$ & $\begin{array}{r}4.4 \\
20.8\end{array}$ & $\begin{array}{r}4.8 \\
25.6\end{array}$ & $\begin{array}{r}5.1 \\
30.7\end{array}$ & $\begin{array}{r}5.6 \\
36.3\end{array}$ \\
\hline
\end{tabular}


ASSUMFTIONS (1982\$)

14285 GFD RATEN CA

CAFITAL COSTS
COMSTR INT. RATE

$\begin{array}{lll} & \text { FERCENT LT DEET } & 75 \% \\ 12.4 \mathrm{MIL} & \text { LT IEDT INT RATE } & 16.0 \% \\ 16.0 \% & \text { LT IIEET FEFAY } & 10 \% \mathrm{YRS} \\ & \text { BANK LINE INT RT } & 16.0 \%\end{array}$

INFLATION RATE

$8.0 \%$ ETH SALE PRICE $1.70 \quad 1 / \mathrm{GAL}$
NET ETH PRICE $.90 \$ / \mathrm{GAL}$ DEG PRICE $170.00 \$ / T O N$ $\begin{array}{lr}\text { DDG PRICE } & 170.00 \mathrm{~S} / \text { TON } \\ \text { COPN COST } & 3.20 \mathrm{~S} / \mathrm{BU}\end{array}$ WOOD COST $10.70 \mathrm{~s} / \mathrm{TON}$

$\begin{array}{llll}\text { ELECTR SALES FRICE } & 5.0 \text { C/KWH } & \text { ETH REAL ESC } & 0.0 \% \\ \text { ELECTK FURCH COST } & 5.0 \text { C } / \text { KWH } & \text { CORN } 8 \text { IDG REAL ESC } & 0.0 \% \\ & & \text { ELECTR KEAL ESC } & 0.0 \% \\ & & \text { WDOD REAL ESC } & 0.0 \%\end{array}$

DAYS REC ETH/DRY AG 30 $\begin{array}{ll}\text { DAYS REC DDG } & 30 \\ \text { DAYS REC ELECTR } & 30\end{array}$

IIAYS PAY CORN DAYS. F'AY WOOD

LFO INUENTORY POLICY

15 DAYS ETH STORAGE

15 DAYS UIIG STORAGE

DAYS. FAY WOOD.

FEI INC TAX

STA.TE INC TAX

STFTE SALES TAX EXEMPT

STATE GASOLINE EXCISE TAX EXEMPT

FEL GASOLINE EXCISE TAX EXEMFT

$46.0 \%$
$4.0 \%$
$0.0 \mathrm{C} / \mathrm{GAL}$
$4.0 \mathrm{C} / \mathrm{GAL}$
$4.0 \mathrm{C} / \mathrm{GAL}$

EQUIP DEFF LITE

EQUIP ITC

BLDG ITC

5 YRS

15 DAYS WOOD STORAGE

RESULTS

ROE $15.0 *$ NPV (25 $\%$ DISC RATE) -2.7 MIL PAYHACK 1992

DEBT COV:

1.1 IN OFER YR 1

1.6 IN OPER YF \&

ENG COSTS \$.6 MIL

INITIAL DEET \$12.4 MIL

MAXIMUM CASH EQUITY

$7.7 \mathrm{MIL}$

ANMUAL SALES:

9.8 MIL GALS ETH

16649 TONS DLI
$0.0 \mathrm{MW} \mathrm{ELECT}$
ANNUAL FLANT FEQS

AT FULL CAFACITY

$$
\begin{aligned}
& 1.9 \text { MIL BU CORN } \\
& 6 \text { MIL KWH }
\end{aligned}
$$

29273 ACRES CORN

$166^{\circ} \mathrm{MIL}$ LE STEAM
16649 HEAD CATTLE 


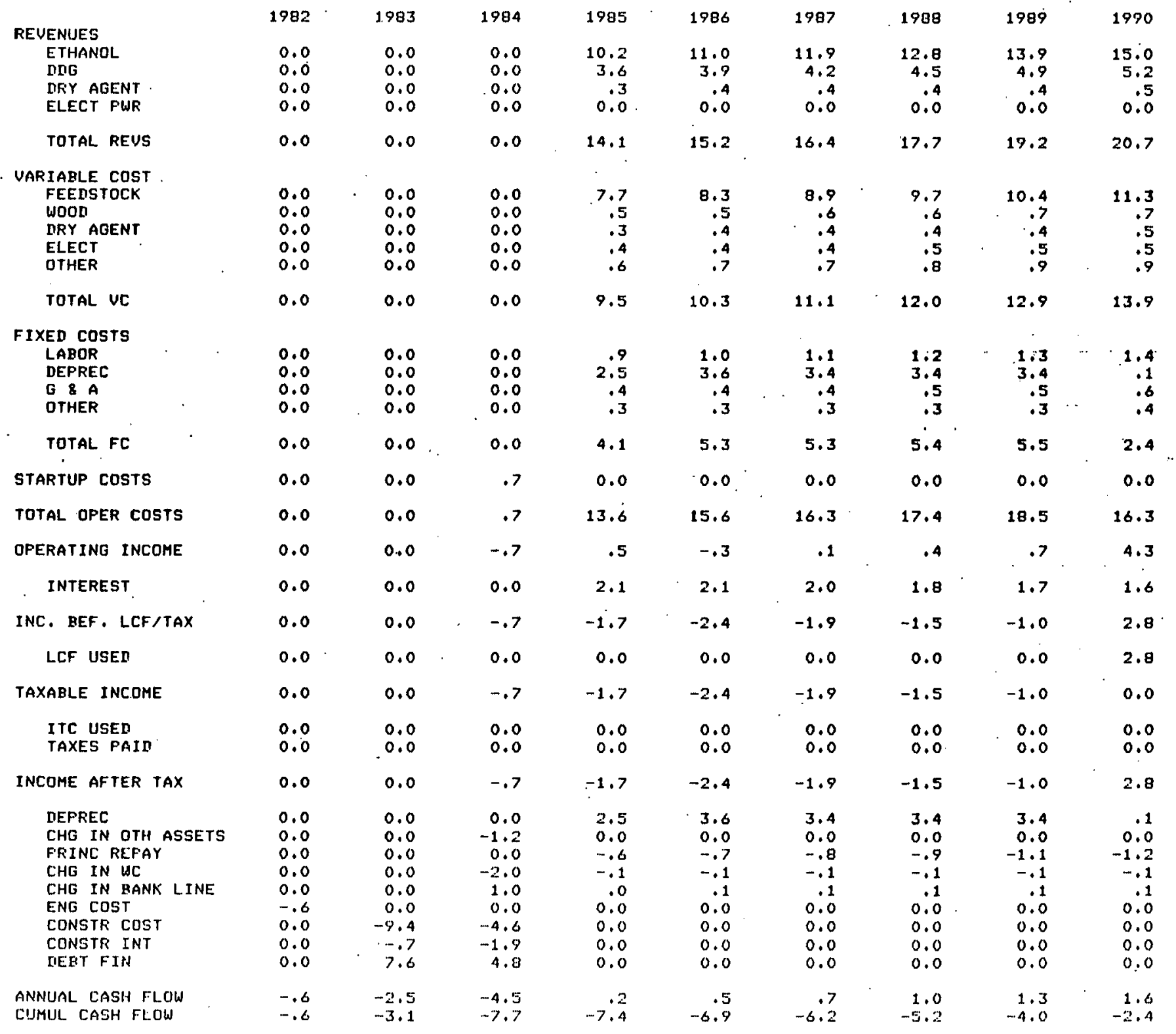


INCOME AND CASH FLOW ( $\$$ MIL)

\begin{tabular}{|c|c|c|c|c|c|c|c|c|c|}
\hline REUENUES & 1991 & $19: 2$ & 1993 & 1994 & 1955 & 1996 & 1997 & 1998 & 1999 \\
\hline ETHANOL & 16.2 & 17.5 & 18.9 & 20.4 & 22.0 & 23.8 & 25.7 & 27.7 & 29.9 \\
\hline nDg & 5.7 & 6.1 & 6.6 & 7.1 & 7.7 & 8.3 & 9.0 & 9.7 & 10.5 \\
\hline DFY AGENT & .5 & .6 & .6 & .7 & .7 & .8 & .8 & .9 & 1.0 \\
\hline ELECT PWR & 0.0 & 0.0 & 0.0 & 0.0 & 0.0 & 0.0 & 0.0 & 0.0 & 0.0 \\
\hline TOTAL REVS & 22.3 & 24.1 & 26.1 & 28.1 & 30.4 & 32.8 & 35.5 & 38,3 & 41.4 \\
\hline \multicolumn{10}{|l|}{ UARIARLE COST } \\
\hline FEEDSTOCK & 12.2 & 13.1 & 14.2 & 15.3 & 16.6 & 17.9 & 19.3 & 20.9 & 22.5 \\
\hline WOOD & .8 & .9 & .9 & 1.0 & 1.1 & 1.2 & 1.3 & 1.4 & 1.5 \\
\hline DRY AGENT & .5 & .6 & .6 & .7 & .7 & .8 & .8 & .9 & 1.0 \\
\hline ELECT & .6 & .6 & .7 & .7 & .8 & .8 & .9 & 1.0 & 1,1 \\
\hline OTHER & 1.0 & 1.1 & 1.2 & 1.3 & 1,4 & 1.5 & 1.6 & 1.7 & 1.9 \\
\hline TOTAL VC & 15.1. & 16.3 & 17.6 & 19.0 & 20.5 & 22.1 & 23.9 & 25.8 & 27.9 \\
\hline \multicolumn{10}{|l|}{ FIXED COSTS } \\
\hline LABOR & 1.5 & 1.6 & 1.7 & 1.9 & 2.0 & 2.2 & 2.4 & 2.6 & 2.8 \\
\hline DEPREC & .1 & 1 & .1 & .1 & .1 & .1 & .1 & .1 & .1 \\
\hline G $8 A$ & .6 & -6 & .7 & .8 & $\cdot .8$ & .9 & 1.0 & 1.0 & 1.1 \\
\hline DTHER & $\cdot .4$ & -4 & .5 & .5 & .5 & .6 & .6 & .7 & .7 \\
\hline TOTAL FC & 2.6 & 2.8 & 3.0 & 3.2 & 3.5 & 3.7 & 4.0 & 4.4 & 4.7 \\
\hline STARTUP COSTS & 0.0 & 0.0 & 0.0 & 0.0 & 0.0 & 0.0 & 0.0 & 0.0 & 0.0. \\
\hline TOTAL OPER COSTS & 17.6 & 19.0 & 20.6 & 22.2 & 24,0 & 25.9 & 27.9 & 30.2 & 32.6 \\
\hline OPERATING INCDME & 4.7 & 5.1 & 5.5 & 6.0 & 6.4 & 7.0 & 7.5 & 8.1 & 8.8 \\
\hline INTEREST & 1.4 & 1.2 & .9 & .6 & .3 & .3 & .3 & .3 & .3 \\
\hline INC. BEF, LCF/TAX & 3.3 & 3.9 & 4.6 & 5.3 & 6.2 & 6.7 & 7.2 & 7.8 & 8.4 \\
\hline LCF USED & 3.3 & 3.0 & 0.0 & 0.0 & 0.0 & 0.0 & 0.0 & 0.0 & 0.0 \\
\hline TAXABLE INCOME & 0.0 & .9 & 4.6 & 5.3 & 6.2 & 6.7 & 7.2 & 7.8 & 8.4 \\
\hline $\begin{array}{l}\text { ITC USED } \\
\text { TAXES FAID }\end{array}$ & $\begin{array}{l}0.0 \\
0.0\end{array}$ & $\begin{array}{l}.5 \\
0.0\end{array}$ & $\begin{array}{l}2.3 \\
0.0\end{array}$ & $\begin{array}{r}.4 \\
2.3\end{array}$ & $\begin{array}{l}0.0 \\
3.1\end{array}$ & $\begin{array}{l}0.0 \\
3.3\end{array}$ & $\begin{array}{l}0.0 \\
3.6\end{array}$ & $\begin{array}{l}0.0 \\
3.9\end{array}$ & $\begin{array}{l}0.0 \\
4.2\end{array}$ \\
\hline INCOME AFTER TAX & 3.3. & 3.9 & 4.6 & 3.0 & 3.1 & 3,3 & 3.6 & 3.9 & 4.2 \\
\hline DEFREC & .1 & .1 & .1 & .1 & .1 & .1 & .1 & .1 & .1 \\
\hline CHG IN OTH ASSETS & 0.0 & 0.0 & 0.0 & 0.0 & 0.0 & 0.0 & 0.0 & 0.0 & 0.0 \\
\hline PRINC REFAY & -1.4 & -1.6 & -1.9 & -2.2 & 0.0 & 0.0 & 0.0 & 0.0 & 0.0 \\
\hline CHG IN WC & -.2 &,- 2 & -.2 & -.2 & -.2 & -.2 &.- .2 & -.3 & -.3 \\
\hline CHG IN BANK LINE & .1 &, 1 & .1 & .1 & .1 & .1 & .1 & .1 & .1 \\
\hline ENG COST & 0.0 & 0,0 & 0.0 & 0.0 & 0.0 & 0.0 & 0.0 & 0.0 & 0.0 \\
\hline CONGTR COST & 0.0 & 0,0 & 0.0 & 0.0 & 0.0 & 0.0 & 0.0 & 0.0 & 0.0 \\
\hline CONSTR INT & 0.0 & 0,0 & 0.0 & 0.0 & 0.0 & 0.0 & 0.0 & 0.0 & 0.0 \\
\hline DEET FIN & 0.0 & 0,0 & 0.0 & 0.0 & 0.0 & 0.0 & 0.0 & 0.0 & 0.0 \\
\hline ANNUAL CASH FLOW & 1.9 & 2,3 & 2.7 & .8 & 3.1 & 3.3 & 3.6 & 3.8 & 1.1 \\
\hline CUMUL CASH FLOW & -.5 & 1,8 & 4.5 & 5.3 & 8,4 & 11.7 & 15.2 & 19.1 & 23.2 \\
\hline
\end{tabular}


ASSUMFTIONS (1982\$)

14205 GPI RATED CAP

\section{ETH SALE PRICE 1.70 \$GAL \\ NET ETH FRICE .90 /GAL \\ DIG PRICE $170.00 \% / T O N$}

CORN COST $2.30 \$ / B U$

$\begin{array}{ll}\text { ELECTR SALES FRICE } & 5.0 \mathrm{C} / \mathrm{KWH} \\ \text { ELECTR FURCH COST } & 5.0 \mathrm{C} / \mathrm{KWH}\end{array}$

$\begin{array}{ll}\text { ETH REAL ESC } & 0.0 \% \\ \text { COFN } 8 \text { DDG REAL ESC } & 0.0 \%\end{array}$

ELECTR REAL ESC $0.0 \%$

DAYS REC ETH/DEY AG 30

$\begin{array}{ll}\text { DAYS REC DDG } & 30 \\ \text { DAYS REC ELECTR } & 30\end{array}$

\section{IIAYS FAY CORN}

IIAYS FAY WOOD

IIAYS FAY WOOD

0
0
0

\section{$46.0 \%$
$4.0 \%$ \\ $0.0 \mathrm{C} / \mathrm{GAL}$ \\ $4.0 \mathrm{C} / \mathrm{GAL}$}

STATE INC TAX

STATE SALES TAX EXERPT

STATE GASOLINE EXCIEE TAX EXEMPT

FED GASOL INE EXCISE TAX EXEMPT

EQUIF DEPR LIFE
EQUIP ITC
BLDG ITC

$5 \cdot$ YRS

$\begin{array}{rl}20 & x \\ 0 & x\end{array}$

RESULTS

ROE $31.7 \%$

MPV (25 \% DISC RATE)

$1.8 \mathrm{MIL}$

PAYBACK

1987

DEBT COV:

1.8 IN OFEK YR 1

2.6 IN OPER YR 6.

ENG costs $8 \quad .6 \mathrm{MIL}$

INITIAL DEBT $\$ 2.4 \mathrm{MIL}$

MAXIMUM CASH EQUITY

IFO INUENTORY POLICY

15 IIAYS ETH STORAGE

15 DAYS CORN STORAG

IS DAYS WOOD STORAGE

ANNUAL SALES:

4.8 MIL GALS ETH

16649 TONS DNG

0.0 MW ELECT

ANNUAL FLANT REQS AT FULL CAFACITY

$$
\begin{aligned}
& 1.9 \mathrm{MIL} \text { BU CORN } \\
& 6 \mathrm{MIL} \text { KWH }
\end{aligned}
$$

29273 ACRES CORN 166 MIL LB STEAM
16649 HEAD CATTLE 
INCOME AND CASH FLOW (\$ MIL)

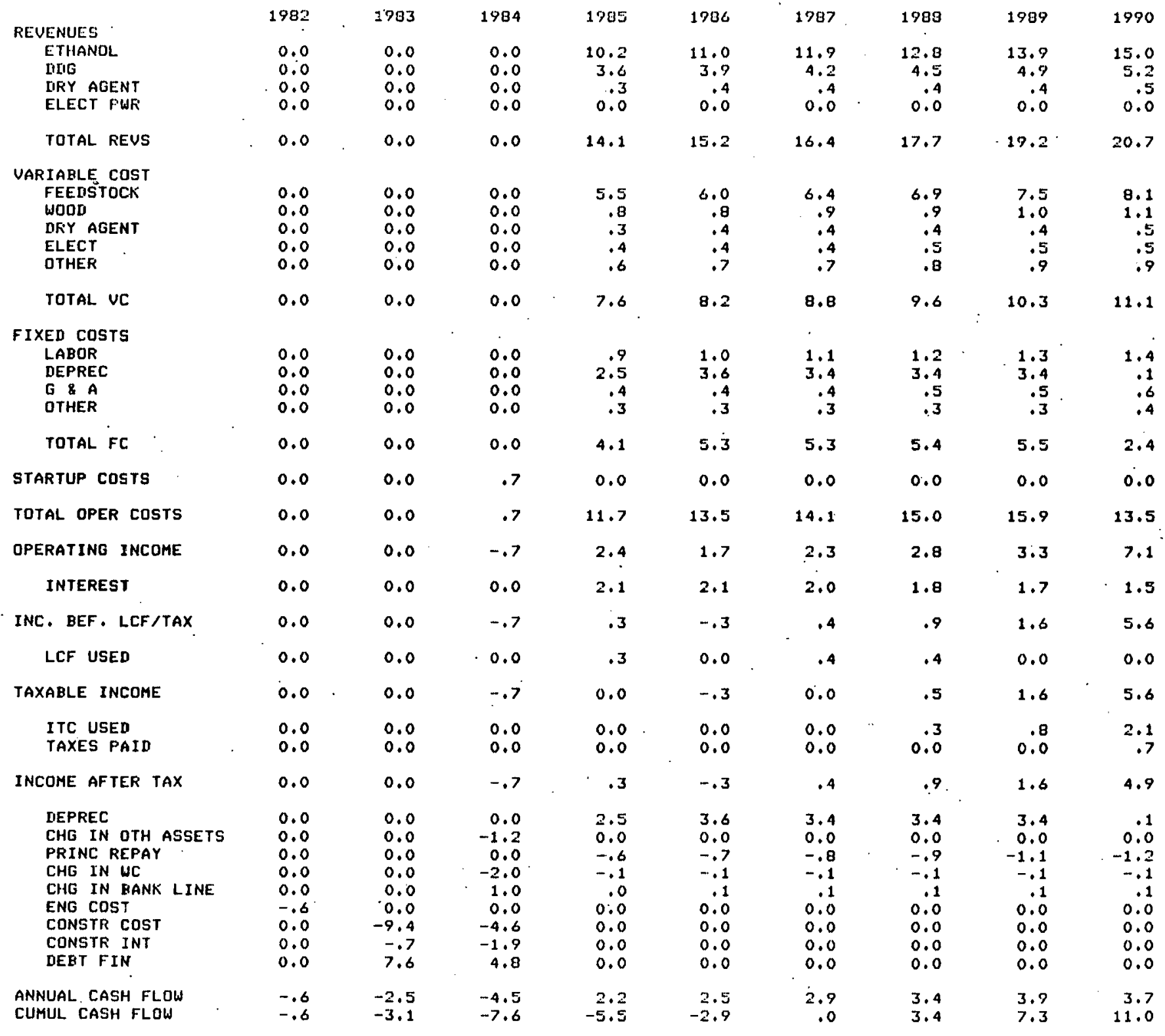


INCOME ANU CASH FLOW (\$ MIL)

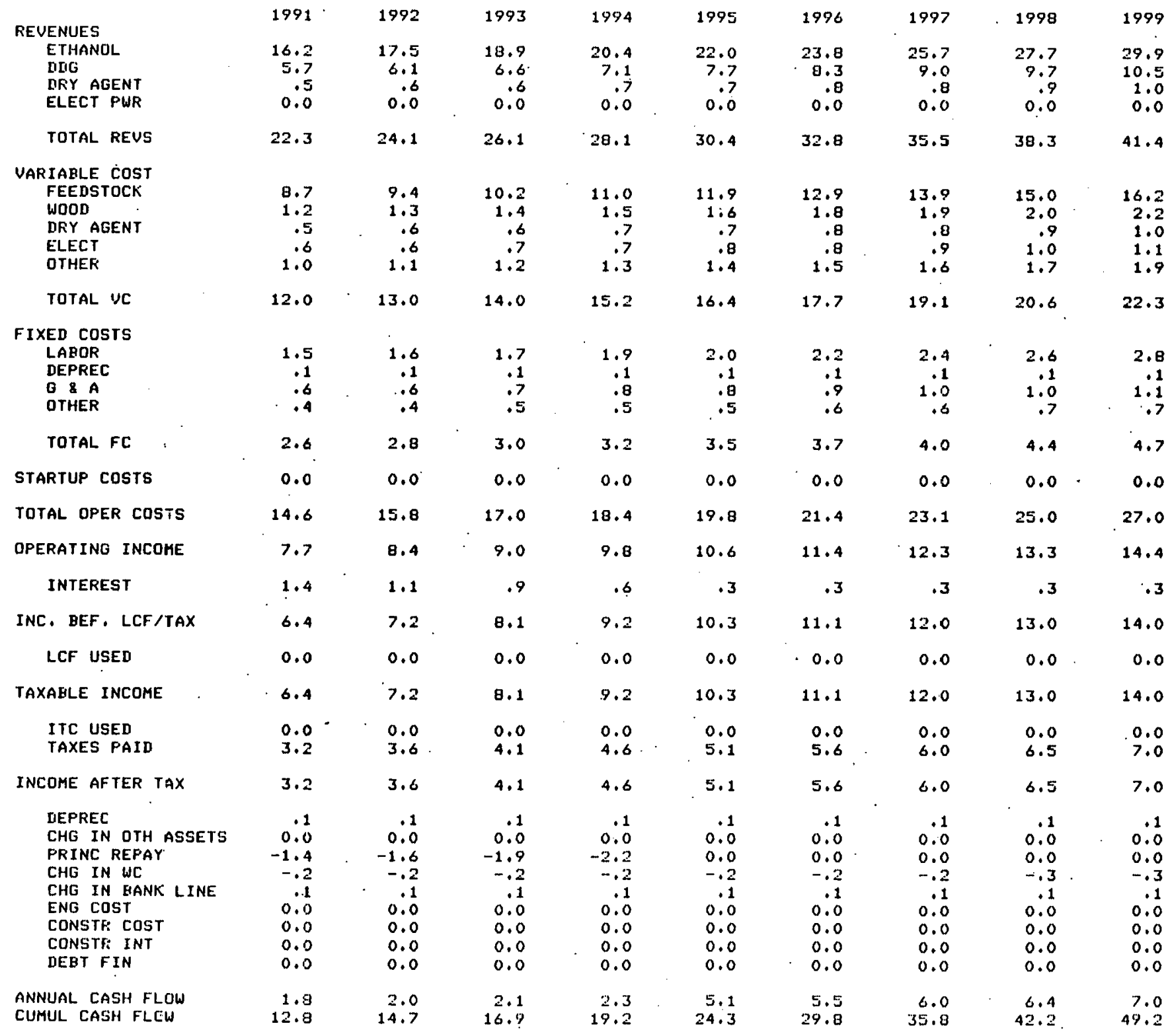


ASGUMFTIONS (1982 \$)

14285 GPD RATED CAF

$100 \%$ ACT CAP
333 LIAYS PER YEAR
$2.5 \mathrm{GAL} E \mathrm{ETH} / \mathrm{BU}$

18 LBS DDG/BU

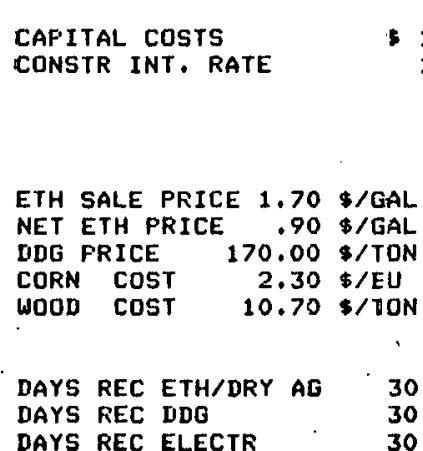

$12.4 \mathrm{MIL}$
$16.0 \%$
GAL
TOL
EU
TON
30
30
30

\section{INFLATION RATE}

CAP COST REAL ESC

$\begin{array}{lll}\text { FERCENT LT DEBT } & 75 & \% \\ \text { LT DEET INT RATE } & 16.0 \% \\ \text { LT IIEBT REPAY } & 10 \% \text { YRS }\end{array}$ BANK LINE INT RT $16.0 \%$
BAS

\section{ELECTR SALES PRICE
ELECTR FURCH CDST}

\section{$\begin{array}{ll}5.0 & \mathrm{C} / \mathrm{KWH} \\ 5.0 \mathrm{C} / \mathrm{KWH}\end{array}$}

ETH REAL ESC

CORN \& DDG REAL. ESC ELECTR REAL ESC WOOD REAL ESC

DAYS FAY CORN DAYS PAY WODD

0
0

\section{LIFO INUENTORY POLICY \\ 15 DAYS ETH STORAGE \\ 15 JAYS CORN STORAGE \\ 15 DAYS DDG STORAOE}

$\begin{array}{llll}\text { FED INC TAX } & 46.0 \% & \text { EQUIF IVEFR LIFE } & 5 \% \text { YRS } \\ \text { STATE INC TAX } & 4.0 \% & \text { ERUIP ITC } & 20 \% \\ \text { STATE SALES TAX EXEMPT } & 0.0 \text { C/GAL } & \text { BLDG ITC } \\ \text { STATE GASOLINE EXCISE TAX EXEMPT } & 4.0 \text { C/GAL } & & 0 \% \\ \text { FED GASOLINE EXCISE TAX EXEMPT } & 4.0 \text { C/GAL } & \end{array}$

\section{RESULTS}

\section{ROE}

DEBT COV:

NFV (25 $\%$ DISC RATE)

$.8 \mathrm{MIL}$

PAYBACK

1988

ENG COSTS \$. .6 MIL

INITIAL DEBT $\$ 12.0$ MIL

MAXIMUM CASH EQUITY

$7.4 \mathrm{MIL}$

ANNUAL SALES:

4.8 MIL GALS ETH

16649 TONS ING

$0.0 \mathrm{MW} \mathrm{ELECT}$

16649 HEAD CATTLE 
INCOME AND CASH FLOW (\$ MIL)

\begin{tabular}{|c|c|c|c|c|c|c|c|c|c|}
\hline REUENUES & 1982 & .1983 & 1984 & 1985 & 1986 & 1987 & 1988 & 1989 & 1990 \\
\hline ETHANOL & 0.0 & 0.0 & 0.0 & 9.4 & 9.8 & 10.3 & 10.8 & $11.4^{\circ}$ & 11.9 \\
\hline nog & 0.0 & 0.0 & 0.0 & 3.3 & 3.4 & 3.6 & 3.8 & 4.0 & 4.2 \\
\hline DRY AGENT & 0.0 & 0.0 & 0.0 & .3 & .3 & .3 & .4 & .4 & $\therefore 4$ \\
\hline ELECT PWR & 0.0 & 0.0 & 0.0 & 0.0 & 0.0 & 0.0 & 0.0 & 0.0 & 0.0 \\
\hline TOTAL REUS & 0.0 & 0.0 & 0.0 & 12.9 & 13.6 & 14.3 & 15.0 & 15.7 & 16.5 \\
\hline UARIARLE COST & & & & & & & & & \\
\hline FEEDSTOCK & 0.0 & 0.0 & 0.0 & 5.1 & 5.3 & 5.6 & 5.9 & 6.2 & 6.5 \\
\hline WOOD & 0.0 & 0.0 & 0.0 & .5 & .5 & .5 & .5 & .6 & .6 \\
\hline DRY AGENT & 0.0 & 0.0 & 0.0 & .3 & .3 & .3 & .4 & .4 & .4 \\
\hline ELECT & 0.0 & 0.0 & 0.0 & .3 & .3 & .4 & .4 & .4 & .4 \\
\hline OTHER & 0.0 & 0.0 & 0.0 & .6 & .6 & .6 & .7 & .7 & .7 \\
\hline TOTAL VC & 0.0 & 0.0 & 0.0 & 6.7 & 7.1 & 7.4 & 7.8 & 8.2 & 8.6 \\
\hline FIXEN COSTS & & & & & & & & & \\
\hline LAROR & 0.0 & 0.0 & 0.0 & .9 & .9 & 1.0 & 1.0 & 1.1 & 1.1 \\
\hline DEPREC & 0.0 & 0.0 & 0.0 & 2.4 & 3.5 & 3.3 & 3.3 & 3.3 & .1 \\
\hline B 8 A & 0.0 & 0.0 & 0.0 & .3 & .4 & .4 & .4 & .4 & $: 4$ \\
\hline OTHER & 0.0 & 0.0 & 0.0 & .2 & $\cdot 2$ & .3 & .3 & .3 & .3 \\
\hline TOTAL FC & 0.0 & 0.0 & 0.0 & 3.9 & 5.0 & 4.9 & $5: 0$ & $5.0 \cdots$ & 1.9 \\
\hline STARTUP COSTS & 0.0 & 0.0 & .7 & 0.0. & 0.0 & 0.0 & 0.0 & 0.0 & 0.0 \\
\hline TOTAL OPER COSTS' & 0.0 & 0.0 & .7 & 10.6 & 12.1 & 12.3 & 12.8 & 13.2 & 10.5 \\
\hline OPERATINO INCOME & 0.0 & 0.0 & -.7 & 2.3 & 1.5 & 1.9 & 2.2 & 2.5 & $\therefore 6: 0$ \\
\hline INTEREST & 0.0 & 0.0 & 0.0 & 2.1 & 2.0 & 1.9 & 1.8 & 1.6. & 1.5 \\
\hline INC, BEF, LCF/TAX & 0.0 & 0.0 & -.7 & .3 & -.5 & .1 & .4 & .9 & 4.5 \\
\hline LCF USEN & 0.0 & 0.0 & 0.0 & .3 & 0.0 & .1 & .4 & .4 & 0.0 \\
\hline TAXABLE INCOME & 0.0 & 0.0 & -.7 & 0.0 & -.5 & 0.0 & 0.0 & .5 & 4.5 \\
\hline $\begin{array}{l}\text { ITC USED } \\
\text { TAXES FAID }\end{array}$ & $\begin{array}{l}0.0 \\
0.0\end{array}$ & $\begin{array}{l}0.0 \\
0: 0\end{array}$ & $\begin{array}{l}0.0 \\
0.0\end{array}$ & $\begin{array}{l}0.0 \\
0.0\end{array}$ & $\begin{array}{l}0.0 \\
0.0\end{array}$ & $\begin{array}{l}0.0 \\
0.0\end{array}$ & $\begin{array}{l}0.0 \\
0.0\end{array}$ & $\begin{array}{r}.2 \\
0.0\end{array}$ & $\begin{array}{r}2.3 \\
0.0\end{array}$ \\
\hline INCOME AFTER TAX . & 0.0 & 0.0 & -.7 & .3 & -.5 & .1 & .4 & .9 & 4.5 \\
\hline DEFREC & 0.0 & 0.0 & 0.0 & 2.4 & 3.5 & 3.3 & 3.3 & 3.3 & .1 \\
\hline CHG IN OTH ASSETS & 0.0 & 0.0 & -1.2 & 0.0 & 0.0 & 0.0 & 0.0 & 0.0 & 0.0 \\
\hline PRINC REFAY & 0.0 & 0.0 & 0.0 & -.6 & -.7 & -.8 & -.9 & -1.0 & -1.2 \\
\hline CHG IN WC & 0.0 & 0.0 & -1.8 & -.1 & -.1 & -.1 & -.1 & -.1 & -.1 \\
\hline CHG IN WANK LINE & 0.0 & 0.0 & .9 & .0 & .0 & .0 & .0 & .0 & .0 \\
\hline ENG $\cos T$ & -.6 & 0.0 & 0.0 & 0.0 & 0.0 & 0.0 & 0.0 & 0.0 & 0.0 \\
\hline CONSTR COST & 0.0 & -9.1 & -4.3 & 0.0 & 0.0 & 0.0 & 0.0 & 0.0 & 0.0 \\
\hline CONSTE INT & 0.0 & -.7 & -1.8 & 0.0 & 0.0 & 0.0 & 0.0 & 0.0 & 0.0 \\
\hline DEET FIN & 0.0 & 7.4 & 4.6 & 0.0 & 0.0 & 0.0 & 0.0 & 0.0 & 0.0 \\
\hline $\begin{array}{l}\text { ANNUAL CASH FLOWW } \\
\text { CUMUL CASH FLOW }\end{array}$ & $\begin{array}{l}-.8 \\
-.8\end{array}$ & $\begin{array}{l}-2.5 \\
-3.1\end{array}$ & -4.3 & $2 \cdot 1$ & $2 \cdot 3$ & 2.6 & 2.8 & 3.1 & 3.4 \\
\hline & -6 & -3.1 & $-7 \cdot 4$ & 5.3 & -2.9 &.- .4 & 2.5 & 5.6 & 9.0 \\
\hline
\end{tabular}


INCOME AND CASH FLOW (\$ MIL)

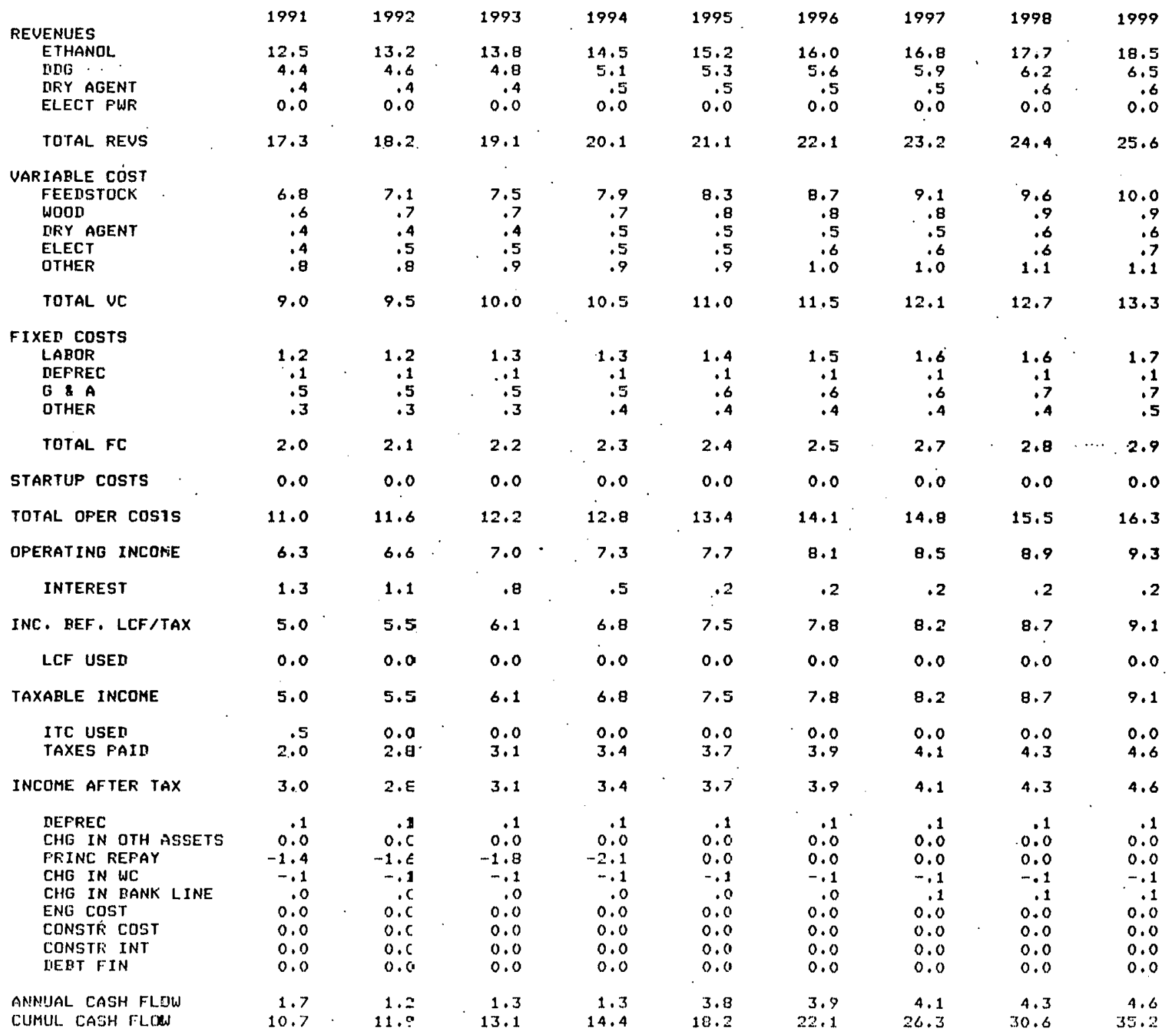




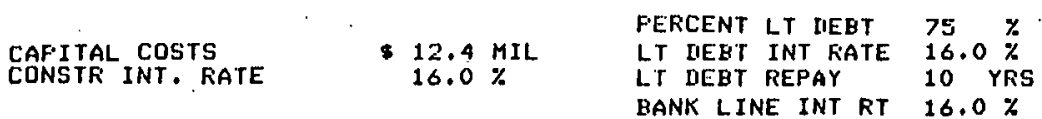

$$
\begin{aligned}
& \begin{array}{l}
\text { INFLATION RATE } \\
\text { CAP COST REAL ESC }
\end{array} \\
& 5.0 \% \frac{\text { INFL }}{5.0 \%}
\end{aligned}
$$$$
\text { LT DEBT REPAY } 10 \text { YRS }
$$

ETH SALE FRICE $1.70 \%$ /GAL NET ETH PRICE $.90 \$ / G A L$ DDG PRICE $170.001 /$ TUN CORN COST $2.30 . / / \mathrm{BU}$ $\begin{array}{ll}\text { ELECTR SALES PRICE } & 5.0 \mathrm{C} / \mathrm{KWH} \\ \text { ELECTR FUKCH COST } & 5.0 \mathrm{C} / \mathrm{KWH}\end{array}$

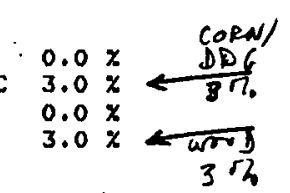

DAYS REC ETH/DRY. AG 30 DAYS REC ELECTR

DAYS PAY CORN DAYS PAY WOOD

15 DAYS WOOD STORAQE

$\begin{array}{llll}\text { FED INC TAX } & 46.0 \% & \text { EQUIP DEFR LIFE } & 5 \text { YRS } \\ \text { STATE INC TAX } & 4.0 x & \text { EQUIP ITC } & 20 \% \\ \text { STATE. SALES TAX EXEMPT } & 0.0 \text { C/GAL } & \text { BLDG ITC } \\ \text { STATE GASOLINE EXCISE TAX EXEMFT } & 4.0 \text { C/GAL } & & 0 \\ \text { FED GASOLINE EXCISE TAX EXEMFT } & 4.0 \text { C/GAL } & \end{array}$

\section{RESULTS}

ROE $25.0 \%$ NFV (25 \% DISC RATE) -.0 MIL PAYBACK 1988

DEBT CON: $\quad 1.7$ IN OPER YR $1 \quad 2.1$ IN OFER YR 6

ENG COSTS $\$ .6 \mathrm{MIL}$ INITIAL IIEBT $\$ 12.0 \mathrm{MIL}$

MAXIMUM CASH EQUITY $\$ 7.4 \mathrm{MIL}$

ANNUAL SALES:

4.8 MIL GALS ETH

16649 TONS DDG

$0.0 \mathrm{HW} \mathrm{ELECT}$ 
INCOME ANI CASH FLOW (\$ MIL)

\begin{tabular}{|c|c|c|c|c|c|c|c|c|c|}
\hline & \multicolumn{9}{|c|}{ REUENUES } \\
\hline ETHANOL & 0.0 & 0.0 & 0.0 & 9.4 & 9.8 & 10.3 & 10.8 & 11.4 & 11.9 \\
\hline $\operatorname{Lng}$ & 0.0 & 0.0 & 0.0 & 3.6 & 3.9 & 4.2 & 4.5 & 4.9 & 5.3 \\
\hline DRY AGENT & 0.0 & 0.0 & 0.0 & .3 & .3 & .3 & .4 & .4 & .4 \\
\hline ELECT FWR & 0.0 & 0.0 & 0.0 & 0.0 & 0.0 & 0.0 & 0.0 & 0.0 & 0.0 \\
\hline TOTAL REUS & 0.0 & 0.0 & 0.0 & 13.2 & 14.0 & 14.8 & 15.7 & 16.6 & 17.6 \\
\hline \multicolumn{10}{|l|}{ UARIABLE $\operatorname{COST}$} \\
\hline FEEDSTOCK & 0.0 & 0.0 & 0.0 & 5.5 & 6.0 & 6.5 & 7.0 & 7.6 & 8.2 \\
\hline wood & 0.0 & 0.0 & 0.0 & .5 & .5 & .5 & .5 & .6 & .6 \\
\hline DRY AGENT & 0.0 & 0.0 & 0.0 & .3 & .3 & .3 & .4 & .4 &. .4 \\
\hline ELECT & 0.0 & 0.0 & 0.0 & .3 & .3 & .4 & .4 & .4 & .4 \\
\hline OTHER & 0.0 & 0.0 & 0.0 & .6 & .6 & .6 & .7 & .7 &. .7 \\
\hline TOTAL UC & 0.0 & 0.0 & 0.0 & 7.2 & 7.7 & 8.3 & B.9 & 9.6 & 10.3 \\
\hline \multicolumn{10}{|l|}{ FIXED CosTS } \\
\hline LABOR & 0.0 & 0.0 & 0.0 & .9 & .9 & 1.0 & 1.0 & 1.1 & 1.1 \\
\hline DEPREC & 0.0 & 0.0 & 0.0 & 2.4 & 3.5 & 3.3 & 3.3 & 3.3 & .1 \\
\hline G 8 A & 0.0 & 0.0 & 0.0 & .3 & .4 & .4 & .4 & .4 & .4 \\
\hline OTHER & 0.0 & 0.0 & 0.0 & .2 & .2 & .3 & .3 & .3 & .3 \\
\hline TOTAL FC & 0.0 & 0.0 & 0.0 & 3.9 & 5.0 & 4.9 & 5.0 & 5.0 & 1.9 \\
\hline STARTUP COSTS & 0.0 & 0.0 & .7 & 0.0 & 0.0 & 0.0 & 0.0 & 0.0 & 0.0 \\
\hline TOTAL OPER CCSTS & 0.0 & 0.0 & .7 & 11.1 & 12.7 & 13.2 & 13.9 & 14.7 & 12.3 \\
\hline OPERATING INCOME & 0.0 & 0.0 & -.7 & .2 .2 & 1.3 & 1.6 & 1.8 & 2.0 & 5.4 \\
\hline INTEREST & 0.0 & 0.0 & 0.0 & 2.1 & 2.0 & 1.9 & 1.8 & 1.6 & 1.5 \\
\hline INC. BEF, LCF/TAX & 0.0 & 0.0 & -.7 & .1 & -.7 & -.3 & .0 & .4 & 3.9 \\
\hline LCF USED & 0.0 & 0.0 & 0.0 & .1 & 0.0 & 0.0 & .0 & .4 & 1.2 \\
\hline TAXABLE INCOHE & 0.0 & 0.0 . & -.7 & 0.0 & -.7 & -.3 & 0.0 & 0.0 & 2.7 \\
\hline $\begin{array}{l}\text { ITC USED } \\
\text { TAXES PAID }\end{array}$ & $\begin{array}{l}0.0 \\
0.0\end{array}$ & $\begin{array}{l}0.0 \\
0.0\end{array}$ & $\begin{array}{l}0.0 \\
0.0\end{array}$ & $\begin{array}{l}0.0 \\
0.0\end{array}$ & $\begin{array}{l}0.0 \\
0.0\end{array}$ & $\begin{array}{l}0.0 \\
0.0\end{array}$ & $\begin{array}{l}0.0 \\
0.0\end{array}$ & $\begin{array}{l}0.0 \\
0.0\end{array}$ & $\begin{array}{l}1.4 \\
0.0\end{array}$ \\
\hline INCOME AFTER TAX & 0.0 & 0.0 & -.7 & .1 & -.7 & -.3 & .0 & .4 & 3.9 \\
\hline DEPREC & .0 .0 & .0 .0 & 0.0 & 2.4 & 3.5 & 3.3 & 3.3 & 3.3 & .1 \\
\hline CHG IN OTH ASSETS & 0.0 & 0.0 & -1.2 & 0.0 & 0.0 & 0.0 & 0.0 & 0.0 & 0.0 \\
\hline PRINC REFAY & 0.0 & 0.0 & 0.0 & -.6 & -.7 & -.8 & -.9 & -1.0 & -1.2 \\
\hline CHG IN WC & 0.0 & 0.0 & -1.9 & -.1 & -.1 & -.1 & -.1 & -.1 &.-.1 \\
\hline CHG IN BANK LINEE & 0.0 & 0.0 & .9 & .0 & .0 & .0 & .0 & .0 & .0 \\
\hline ENG $\cos T$ & -.6 & 0.0 & 0.0 & 0.0 & 0.0 & 0.0 & 0.0 & 0.0 & 0.0 \\
\hline CONSTR COST & 0.0 & -9.1 & -4.3 & 0.0 & 0.0 & 0.0 & 0.0 & 0.0 & 0.0 \\
\hline CONSTR INT & 0.0 & -.7 & -1.8 & 0.0 & 0.0 & 0.0 & 0.0 & 0.0 & .0 .0 \\
\hline DEET FIN & 0.0 & 7.4 & 4.6 & 0.0 & 0.0 & 0.0 & 0.0 & 0.0 & 0.0 \\
\hline $\begin{array}{l}\text { ANNUAL CASH FLOW } \\
\text { CUMUL CASH FLOW }\end{array}$ & $\begin{array}{l}-.6 \\
-.6\end{array}$ & $\begin{array}{l}-2.5 \\
-3.1\end{array}$ & $\begin{array}{l}-4.3 \\
-7.4\end{array}$ & $\begin{array}{r}1.9 \\
-5.5\end{array}$ & $\begin{array}{r}2.1 \\
-3.4\end{array}$ & $\begin{array}{r}2.3 \\
-1.1\end{array}$ & $\begin{array}{l}2.4 \\
1.3\end{array}$ & $\begin{array}{l}2.6 \\
3.9\end{array}$ & $\begin{array}{l}2.8 \\
6.7\end{array}$ \\
\hline
\end{tabular}


INCOME ANI CASH FLOW (\$ MIL)

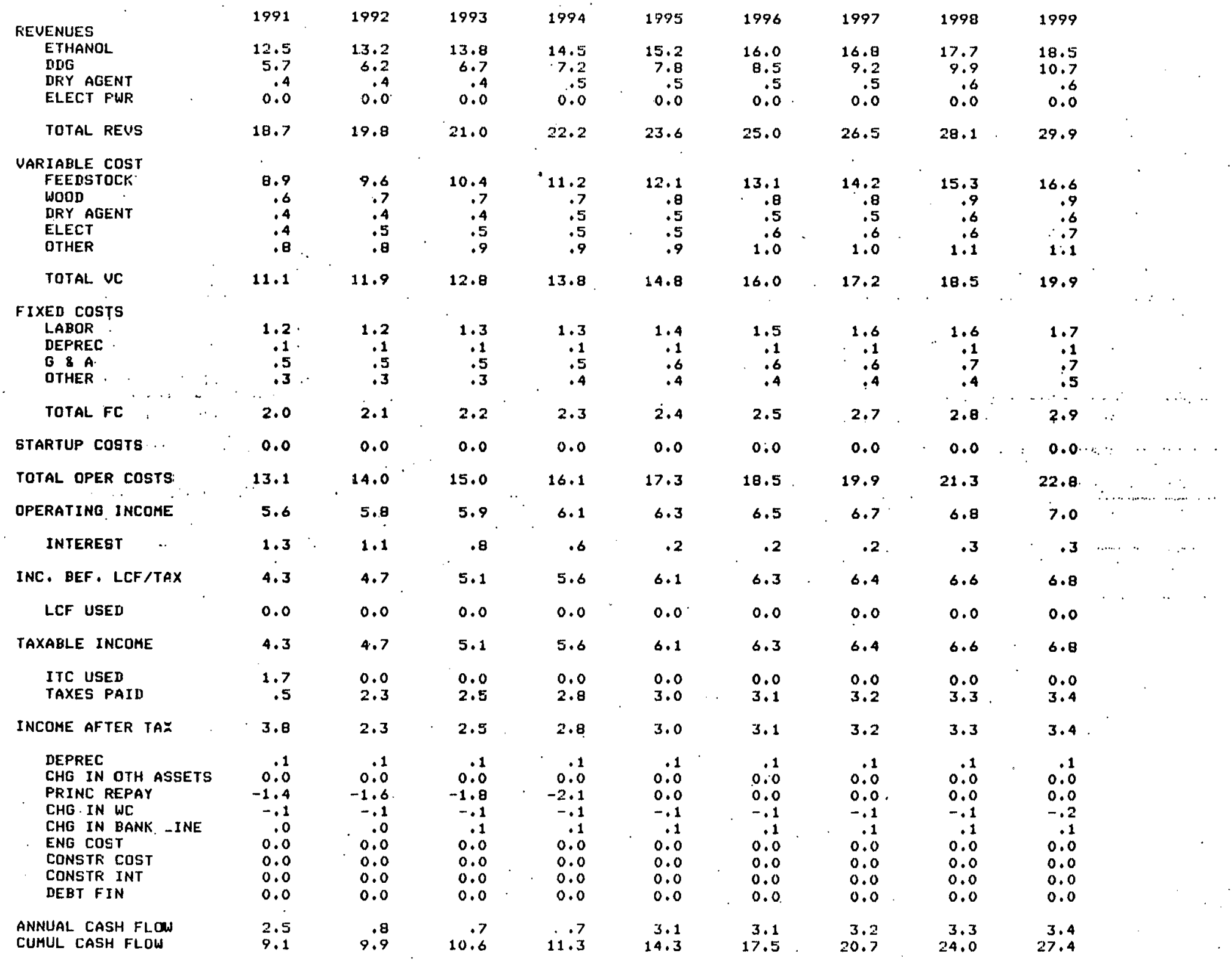


ASSUMPTIONS (1982 \$)

14285 GFD RATES CAP

$100 \%$ ACT CAF
333 DAYS PER YEAR
$2.5 \mathrm{GAL}$ ETH/BU

$18 \mathrm{LBS} \mathrm{DDG} / \mathrm{BU}$

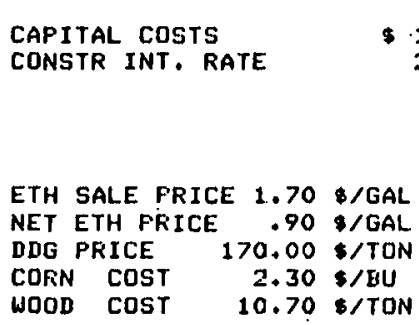

$$
\begin{array}{lll} 
& \text { PERCENT LT DEBT } & 75 \% \\
12.4 \mathrm{MIL} & \text { LT DEBT INT RATE } & 20.0 \% \\
20.0 \% & \text { LT DEBT FEPAY } & 10 \% \text { YRS } \\
& \text { BANK LINE INT RT } & 20.0 \%
\end{array}
$$

INFLATION RATE

CAF COST

$5.0 \%$
ELECTR SALES PRICE
ELECTR PURCH COST

$$
5.0 \mathrm{C} / \mathrm{KWH}
$$

ETH REAL ESC

CORN D DDG REAL ESC $0.0 \%$

ELECTR REAL ESC $0.0 \%$ WOOD REAL ESC

DAYS REC ETH/DRY AG

DAYS REC DDG

30
30
30

DAYS PAY CORN DAYS FAY WOOD

LIFO INUENTORY POLICY

15 DAYS ETH STORAGE

15 DAYS CORN STORAGE

15 DAYS DDB STORAGE

FED INC TAX

STATE INC TAX

STATE SALES TAX EXEMP

TAX EXEMPT

FED GASOLINE EXCISE TAX EXEMPT

$\begin{array}{rl}46.0 & \chi \\ 4.0 & x \\ 0.0 & C / G A L \\ 4.0 & C / G A L \\ 4.0 & C / G A L\end{array}$

EQUIP DEFR LIFE

EQUIP ITC

BLDG ITC

5 YRS

$20 x$

RESULTS

ROE $29.9 \times$ NPV $(25 \times$ DISC RATE) $1.4 \mathrm{MIL}$ FAYBACK 1998

DEBT COU: 1.6 IN OFER YR 1 2.3 IN OPER YR 6

ENG COSTS \$ .6 MIL INITIAL DEBT \$12.9 MIL

MAXIMUM CASH EQUITY $7.8 \mathrm{MIL}$

ANNUAL SALES:

$4.0 \mathrm{MIL}$ GALS ETH

16649 TONS DDG

$0.0 \mathrm{MW} \mathrm{ELECT}$ 


\begin{tabular}{|c|c|c|c|c|c|c|c|c|c|}
\hline FEUENUES & 1982 & $15 \theta 3$ & 1984 & 1985 & 1986 & 1907 & 1988 & 1989 & 1990 \\
\hline $\begin{array}{l}\text { ETHANOL } \\
\text { DING AGENT } \\
\text { IIRY ACT PWK } \\
\text { ELECT PWT }\end{array}$ & $\begin{array}{l}0.0 \\
0.0 \\
0.0 \\
0.0\end{array}$ & $\begin{array}{l}c .0 \\
c .0 \\
0.0 \\
0.0\end{array}$ & $\begin{array}{l}0.0 \\
0.0 \\
0.0 \\
0.0\end{array}$ & $\begin{array}{r}10.2 \\
3.6 \\
.3 \\
0.0\end{array}$ & $\begin{array}{r}11.0 \\
3.9 \\
.4 \\
0.0\end{array}$ & $\begin{array}{r}11.9 \\
4.2 \\
.4 \\
0.0\end{array}$ & $\begin{array}{r}12.8 \\
4.5 \\
.4 \\
0.0\end{array}$ & $\begin{array}{r}23.9 \\
4.9 \\
0.4 \\
0.0\end{array}$ & $\begin{array}{r}15.0 \\
5.2 \\
.5 \\
0.0\end{array}$ \\
\hline TOTAL REUS & 0.0 & 0.0 & 0.0 & 14.1 & 15.2 & 16.4 & 17.7 & 19.2 & 20.7 \\
\hline $\begin{array}{l}\text { UARIABLE COST } \\
\text { FEEDSTOCK } \\
\text { WOOD } \\
\text { DRY AGENT } \\
\text { ELECT } \\
\text { OTHER }\end{array}$ & $\begin{array}{l}0.0 \\
0.0 \\
0.0 \\
0.0 \\
0.0\end{array}$ & $\begin{array}{l}0.0 \\
0.0 \\
0.0 \\
0.0 \\
0.0\end{array}$ & $\begin{array}{l}0.0 \\
0.0 \\
0.0 \\
0.0 \\
0.0\end{array}$ & $\begin{array}{r}5.5 \\
.5 \\
.3 \\
.4 \\
.6\end{array}$ & $\begin{array}{r}6.0 \\
.5 \\
.4 \\
.4 \\
.7\end{array}$ & $\begin{array}{l}6.4 \\
.6 \\
.4 \\
.4 \\
.7\end{array}$ & $\begin{array}{r}6.9 \\
.6 \\
.4 \\
.5 \\
.8\end{array}$ & $\begin{array}{r}7.5 \\
.7 \\
.4 \\
.5 \\
.9\end{array}$ & $\begin{array}{r}8.1 \\
.7 \\
.5 \\
.5 \\
.9\end{array}$ \\
\hline TOTAL VC & 0.0 & 0.0 & 0.0 & 7.3 & 7.9 & 8.6 & 9.2 & 10.0 & 10.8 \\
\hline $\begin{array}{l}\text { FIXED COSTS } \\
\text { LABOR } \\
\text { DEPREC } \\
\text { G \& A } \\
\text { OTHER }\end{array}$ & $\begin{array}{l}0.0 \\
0.0 \\
0.0 \\
0.0\end{array}$ & $\begin{array}{l}0.0 \\
0.0 \\
0.0 \\
0.0\end{array}$ & $\begin{array}{l}0.0 \\
0.0 \\
0.0 \\
0.0\end{array}$ & $\begin{array}{r}.9 \\
2.6 \\
.4 \\
.3\end{array}$ & $\begin{array}{r}1.0 \\
3.7 \\
.4 \\
.3\end{array}$ & $\begin{array}{r}1.1 \\
3.6 \\
.4 \\
.3\end{array}$ & $\begin{array}{r}1.2 \\
3.5 \\
.5 \\
.3\end{array}$ & $\begin{array}{r}1.3 \\
3.5 \\
.5 \\
.3\end{array}$ & $\begin{array}{r}1.4 \\
.1 \\
.6 \\
.4\end{array}$ \\
\hline TOTAL FC & 0.0 & 0.0 & 0.0 & 4.2 & 5.4 & 5.4 & 5.5 & 5.7 & 2.4 \\
\hline STARTUP COSTS & 0.0 & 2.0 & .7 & 0.0 & 0.0 & 0.0 & 0.0 & 0.0 & 0.0 \\
\hline TOTAL OPER COSTS & 0.0 & 0.0 & .7 & 11.5 & 13.4 & 14.0 & 14.8 & 15.7 & 13.2 \\
\hline OPERATING INCOME & 0.0 & 0.0 & -.7 & 2.6 & 1.8 & 2.5 & 3.0 & 3.5 & 7.5 \\
\hline INTEREST & 0.0 & 0.0 & 0.0 & 2.8 & 2.7 & 2.6 & 2.4 & 2.3 & 2.1 \\
\hline INC. BEF. LCF/TAX & 0.0 & 0.0 &.,- 7 & -.2 & -.8 & -.1 & .5 & 1.2 & 5.4 \\
\hline LCF USED & 0.0 & 0.0 & 0.0 & 0.0 & .0 .0 & 0.0 & .5 & 1.2 & .1 \\
\hline TAXABLE INCOME & 0.0 & 0.0 & -.7 & -.2 & -.8 & -.1 & 0.0 & 0.0 & 5.3 \\
\hline $\begin{array}{l}\text { ITC USED } \\
\text { TAXES FAID }\end{array}$ & $\begin{array}{l}0.0 \\
0.0\end{array}$ & $\begin{array}{l}0.0 \\
0.0\end{array}$ & $\begin{array}{l}0.0 \\
0.0\end{array}$ & $\begin{array}{l}0.0 \\
0.0\end{array}$ & $\begin{array}{l}0.0 \\
0.0\end{array}$ & $\begin{array}{l}0.0 \\
0.0\end{array}$ & $\begin{array}{l}0.0 \\
0.0\end{array}$ & $\begin{array}{l}0.0 \\
0.0\end{array}$ & $\begin{array}{l}2.6 \\
0.0\end{array}$ \\
\hline INCOME AFTER TAX & $0: 0$ & 0.0 & -.7 & -.2 & -.8 & -.1 & .5 & 1.2 & 5.4 \\
\hline $\begin{array}{l}\text { DEFREC } \\
\text { CHG IN OTH ASSETS } \\
\text { PRINC REPAY } \\
\text { CHG IN WC } \\
\text { CHG IN HANK LINE } \\
\text { ENG COST } \\
\text { CONSTR COST } \\
\text { CONSTR IN:T } \\
\text { DEBT FIN }\end{array}$ & $\begin{array}{l}0.0 \\
0.0 \\
0.0 \\
0.0 \\
0.0 \\
-.0 \\
0.0 \\
0.0 \\
0.0\end{array}$ & $\begin{array}{r}0.0 \\
0.0 \\
0.0 \\
0.0 \\
0.0 \\
0.0 \\
-9.4 \\
-.9 \\
7.7\end{array}$ & $\begin{array}{r}0.0 \\
-1.2 \\
0.0 \\
-2.0 \\
1.0 \\
0.0 \\
-4.6 \\
-2.3 \\
5.2\end{array}$ & $\begin{array}{l}2.6 \\
0.0 \\
-.5 \\
-.1 \\
.0 \\
0.0 \\
0.0 \\
0.0 \\
0.0\end{array}$ & $\begin{array}{l}3.7 \\
0.0 \\
-.0 \\
-.1 \\
.1 \\
0.0 \\
0.0 \\
0.0 \\
0.0\end{array}$ & $\begin{array}{l}3.6 \\
0.0 \\
-.7 \\
-.1 \\
.1 \\
0.0 \\
0.0 \\
0.0 \\
0.0\end{array}$ & $\begin{array}{l}3.5 \\
0.0 \\
-.9 \\
-.1 \\
.1 \\
0.0 \\
0.0 \\
0.0 \\
0.0\end{array}$ & $\begin{array}{r}3.5 \\
0.0 \\
-1.0 \\
-.1 \\
.1 \\
0.0 \\
0.0 \\
0.0 \\
0.0\end{array}$ & $\begin{array}{r}0.1 \\
0.0 \\
-1.2 \\
-.1 \\
.1 \\
0.0 \\
0.0 \\
0.0 \\
0.0\end{array}$ \\
\hline $\begin{array}{l}\text { ANNUAL CASH FLOW } \\
\text { CUMUL CASH FLOW }\end{array}$ & $\begin{array}{l}-.6 \\
-.6\end{array}$ & $\begin{array}{l}-2.6 \\
-3.2\end{array}$ & $\begin{array}{l}-4.6 \\
-7.8\end{array}$ & $\begin{array}{r}1.9 \\
-5.9\end{array}$ & $\begin{array}{r}2.3 \\
-3.7\end{array}$ & $\begin{array}{r}2.7 \\
-1.0\end{array}$ & $\begin{array}{l}3.2 \\
2.2\end{array}$ & $\begin{array}{l}3.7 \\
5.8\end{array}$ & $\begin{array}{r}4.2 \\
10.0\end{array}$ \\
\hline
\end{tabular}




\begin{tabular}{|c|c|c|c|c|c|c|c|c|c|}
\hline REVENUES & 1991 & 1992 & 1993 & 1994 & 1995 & 1996 & 1997 & 1998 & 1999 \\
\hline ETHANOL & 16.2 & 17.5 & 18.9 & 20.4 & 22.0 & $23, \theta$ & 25.7 & 27.7. & 29.9 \\
\hline & 5.7 & 6.1 & 6.6 & 7.1 & 7.7 & 8.3 & 9.0 & 9.7 & 10.5 \\
\hline DEY AGENT & .5 & .6 & .6 & .7 & .7 &.$\theta$ & .8 & .9 & 1.0 \\
\hline ELECT PiRR & 0.0 & 0.0 & 0.0 & 0.0 & 0.0 & 0.0 & 0.0 & 0.0 & 0.0 \\
\hline TOTAL REUS & 22.3 & 24.1 & 26.1 & 28.1 & $30: 4$ & 32.8 & 35.5 & 38.3 & 41.4 \\
\hline \multicolumn{10}{|l|}{ VARIABLE COST } \\
\hline FEEDSTOCK & 8.7 & 9.4 & 10.2 & 11.0 & 11.9 & 12.9 & 13.9 & 15.0 & 16.2 \\
\hline WOOD & .8 & .9 & .9 & 1.0 & 1.1 & 1.2 & 1.3 & 1.4 & 1.5 \\
\hline DRY AGENT & .5 & .6 & .6 & .7 & .7 & .8 & 8 & .9 & 1.0 \\
\hline ELECT & .6 & .6 & .7 & .7 & .8 & .8 & .9 & 1.0 & 1.1 \\
\hline OTHER & 1.0 & 1.1 & 1.2 & 1.3 & 1.4 & 1.5 & 1.6 & 1.7 & 1.9 \\
\hline TOTAL VC & 11.6 & 22.6 & 13.6 & 14.7 & 15.8 & 17.1 & 18.5 & 20.0 & 21.5 \\
\hline \multicolumn{10}{|l|}{ FIXED COSTS } \\
\hline LABOR & 1.5 & 1.6 & 1.7 & 1.9 & 2.0 & 2.2 & 2.4 & 2.6 & 2.8 \\
\hline DEPREC & .1 & +1 & .1 & .1 & 1 & .1 & $\therefore 1$ & .1 & .1 \\
\hline $\begin{array}{l}G \& \text { \& A } \\
\text { OTHER }\end{array}$ & .6 & .6 & .7 & .8 &.$\theta$ & .9 & 1.0 & 1.0 & 1.1 \\
\hline UIHER & $\cdot 4$ & $\cdot 4$ & • & .5 & .5 & .6 & .6 & .7 & .7 \\
\hline TOTAL FC & 2.6 & 2.8 & 3.0 & 3.2 & 3.5 & 3.7 & 4.0 & 4.4 & 4.7 \\
\hline STARTUP COSTS & 0.0 & 0.0 & 0.0 & 0.0 & 0.0 & 0.0 & 0.0 & 0.0 & 0.0 \\
\hline TOTAL OPER COSTS & 14.2 & 15.3 & 16.6 & 17.9 & 19.3 & 20.9 & 22.5 & 24.3 & 26.2 \\
\hline OPERATING IMCOME & 8.1 & 8.8 & 9.5 & 10.3 & 11.1 & 12.0 & 12.9 & 14.0 & 15.1 \\
\hline INTEREST & 1.9 & 1.6 & 1.2 & .8 & .3 & .4 & .4 & .4 & .4 \\
\hline INC. BEF. LCF/TAX. & 6.3 & 7.2 & 8.3 & 9.4 & 10.8 & 11.6 & 12.6 & 13.6 & 14.7 \\
\hline LCF USED & 0.0 & 0.0 & 0.0 & 0.0 & 0.0 & 0.0 & 0.0 & 0.0 & 0.0 \\
\hline TAXABLE INCOHE & 6.3 & 7.2 & 8.3 & 9.4 & 10.8 & 11.6 & 12.6 & 13.6 & 14,7 \\
\hline $\begin{array}{l}\text { ITC USED } \\
\text { TAXES FAJD }\end{array}$ & 2.6 & $\begin{array}{l}2.0 \\
3.6\end{array}$ & $\begin{array}{l}0.0 \\
4.1\end{array}$ & $\begin{array}{l}0.0 \\
4.7\end{array}$ & $\begin{array}{l}0.0 \\
5.4\end{array}$ & $\begin{array}{l}0.0 \\
5.8\end{array}$ & $\begin{array}{l}0.0 \\
6.3\end{array}$ & $\begin{array}{l}0.0 \\
6.8\end{array}$ & $\begin{array}{l}0.0 \\
7.3\end{array}$ \\
\hline INCOHE AFTEF TAX & 3.8 & 3.6 & 4,1 & 4.7 & 5.4 & 5.8 & 6.3 & 6.8 & 7.3 \\
\hline DEFREC & .1 & .1 & .1 & $\cdot 1$ & $\cdot 1$ & .1 & $\therefore 1$ & $\cdot 1$ & $\cdot 1$ \\
\hline CHG IN OTH ASSETS & 0.0 & 0.0 & 0.0 & 0.0 & 0.0 & 0.0 & 0.0 & 0.0 & 0.0 \\
\hline FRINC REFAAY & -1.5 & -1.8 & -2.1 & -2.6 & 0.0 & 0.0 & 0.0 & 0.0 & 0.0 \\
\hline CHG IN WC & -.2 & -.2 & -.2 & -.2 & -.2 & -.2 & -.2 & -.3 & -.3 \\
\hline CHG IN BANK LINE & .1 & .1 & .1 & .1 & .1 & .1 & .1 & .1 & .1 \\
\hline ENG $\cos \mathrm{T}$ & 0.0 & 2.0 & 0.0 & 0.0 & 0.0 & 0.0 & 0.0 & 0.0 & 0.0 \\
\hline CONSTR CUST & 0.0 & 0.0 & 0.0 & 0.0 & 0.0 & 0.0 & 0.0 & 0.0 & 0.0 \\
\hline CONSTR INT & 0.0 & 0.0 & 0.0 & 0.0 & 0.0 & 0.0 & 0.0 & 0.0 & 0.0 \\
\hline DEET FIN & 0.0 & 0.0 & 0.0 & 0.0 & 0.0 & 0.0 & 0.0 & 0.0 & 0.0 \\
\hline ANNUAL CASH FLOW & 3 & $\therefore 8$ & .0 & 2.1 & 5.4 & 5.8 & 6.2 & 6.7 & 7.3 \\
\hline CUMUL CASH FLOW & 12.3 & $1+.1$ & 16.1 & 18.3 & 23.6 & 29.4 & 35.6 & 42.4 & 49.6 \\
\hline
\end{tabular}


ASSUMFTIONS (1992 \$)

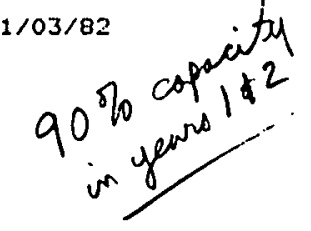$$
\text { - }
$$

CAPITAL COSTS
CONSTR INT. RATE

\section{$12.4 \mathrm{MIL}$
$16.0 \%$}

$\begin{array}{ll}\text { PERCENT LT DEBT } & 75 \% \\ \text { LT DEBT INT RATE } & 16.0 \% \\ \text { LT DEBT REFAY } & 10 \% \text { YRS } \\ \text { BANK LINE INT RT } & 16.0 \%\end{array}$

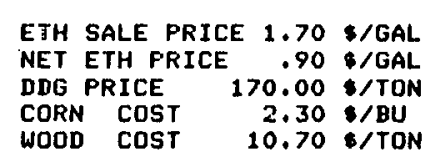

ELECTR SALES FRICE

ELECTKR SALES FRICE
ELECTR PURCH COST
$5,0 \mathrm{C} / \mathrm{KWH}$
$5.0 \mathrm{C} / \mathrm{KWH}$

INFLATION RATE

CAP COST REAL ESC

$8.0 \%$

$5.0 *$
18 LBS DDG/BU
DAYS PAY CORN

DAYS FAY WOOD

DAYS REC ETH/DRY AG

DAYS REC UUG

30
30
30

\section{FED INC TAX \\ STATE INC TAX \\ STATE SALES TAX EXEMPT \\ STATE GASOLINE EXCISE TAX EXEMPT}

FED GASOLINE EXCISE TAX EXEMPT

$\begin{array}{rl}46.0 & \% \\ 4.0 & Z \\ 0.0 & \mathrm{C} / \mathrm{GAL} \\ 4.0 \mathrm{C} / \mathrm{GAL} \\ 4.0 \mathrm{C} / \mathrm{GAL}\end{array}$

RESULTS

ROE $31.7 x$

HPV ( $25 \%$ DISC RATE)

$1.9 \mathrm{MIL}$

FAYBACK

1988

DEET COU:

1.7 IN OFER YR 1

2.7 IN OFER YR 6

ENG COSTS .6 MIL

INITIAL DEBT \$12.4 MIL

MAXIMUM CASH EQUITY $\$ 7.5 \mathrm{MIL}$

AMNUAL SALES:

4.8 MIL GALS ETH

16649 TONS ING

$0.0 \mathrm{KW} \mathrm{ELECT}$

AMNUAL PLANT RERS

AT FULL CAFACITY

1.9 MIL BU CORN
6 MIL KWH

29273 ACRES CDFN

166 MIL LB STEAM

$\begin{array}{ll}\text { ETH REAL ESC } & 0.0 \% \\ \text { CORN DDG REAL ESC } & 0.0 \% \\ \text { ELECTR REAL ESC } & 0.0 \% \\ \text { WOOD REAL ESC } & 0.0 \%\end{array}$

LIFO INUENTORY POLICY

15 DAYS ETH STDRAGE

15 DAYS CDRN STORAGE

15 DAYS DDG STORAGE

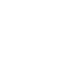

5 YRS

0
0
0 


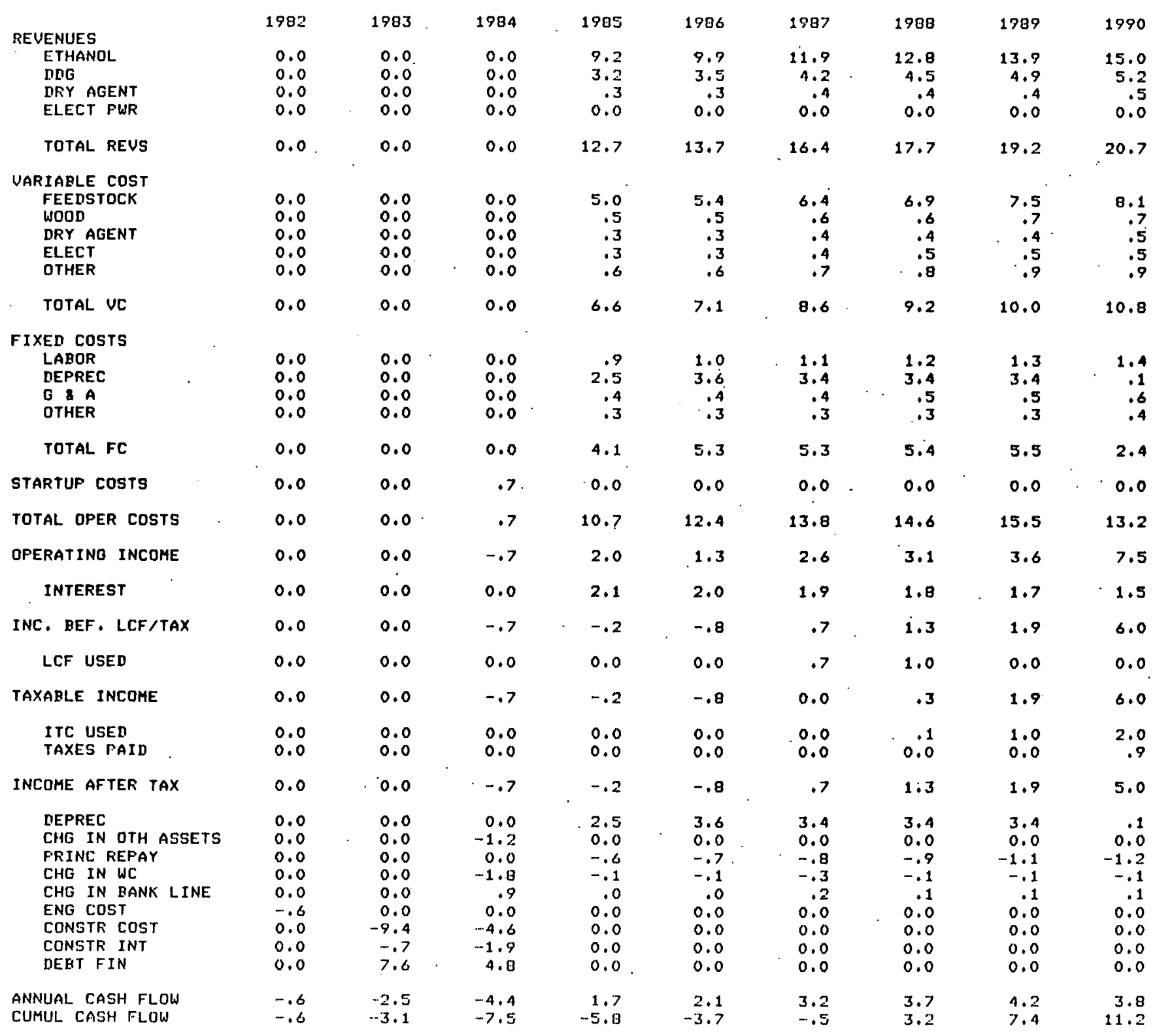




\begin{tabular}{|c|c|c|c|c|c|c|c|c|c|}
\hline REUENUES & 1991 & 1992 & 1993 & 1994 & 1995 & 1996 & 1997 & 19.98 & 1999 \\
\hline $\begin{array}{l}\text { ETHANOL } \\
\text { DDG } \\
\text { DRY AGENT } \\
\text { ELECT FWR }\end{array}$ & $\begin{array}{r}16.2 \\
5.7 \\
.5 \\
0.0\end{array}$ & $\begin{array}{r}17.5 \\
6.5 \\
0.0 \\
0.0\end{array}$ & $\begin{array}{r}18.9 \\
6.6 \\
0.6 \\
0.0\end{array}$ & $\begin{array}{r}20.4 \\
7.1 \\
.7 \\
0.0\end{array}$ & $\begin{aligned} 22.0 \\
7.7 \\
.7 \\
0.0\end{aligned}$ & $\begin{array}{r}23.8 \\
8.3 \\
.8 \\
0.0\end{array}$ & $\begin{array}{r}25.7 \\
.9 .0 \\
.8 \\
0.0\end{array}$ & $\begin{array}{r}27.7 \\
9.7 \\
0.9 \\
0.0\end{array}$ & $\begin{array}{r}29.9 \\
10.5 \\
1.0 \\
0.0\end{array}$ \\
\hline TOTAL REUS & 22.3 & 24.5 & 26.1 & 28.1 & 30.4 & 32.8 & 35.5 & 38.3 & 41.4 \\
\hline $\begin{array}{l}\text { VARIARLE COST } \\
\text { FEERSTOCK } \\
\text { WOOD } \\
\text { DRY AGENT } \\
\text { ELECT } \\
\text { OTHER }\end{array}$ & $\begin{array}{r}8.7 \\
.8 \\
.5 \\
.6 \\
1.0\end{array}$ & $\begin{array}{r}9.4 \\
.9 \\
.6 \\
.0 \\
1.1\end{array}$ & $\begin{array}{r}10.2 \\
.9 \\
.6 \\
.7 \\
1.2\end{array}$ & $\begin{array}{r}11.0 \\
1.0 \\
.7 \\
.7 \\
1.3\end{array}$ & $\begin{array}{r}11.9 \\
1.1 \\
.7 \\
.8 \\
1.4\end{array}$ & $\begin{array}{r}12.9 \\
1.2 \\
.8 \\
.8 \\
1.5\end{array}$ & $\begin{array}{r}13.9 \\
1.3 \\
.8 \\
.9 \\
1.6\end{array}$ & $\begin{array}{r}15.0 \\
1.4 \\
1.9 \\
1.0 \\
1.7\end{array}$ & $\begin{array}{r}16.2 \\
1.5 \\
1.0 \\
1.1 \\
1.9\end{array}$ \\
\hline TOTAL UC & 11.6 & 12.5 & 13.6 & 14.7 & 15.8 & 17.1 & 18.5 & 20.0 & 21.5 \\
\hline $\begin{array}{l}\text { FIXED COSTS } \\
\text { LABOR } \\
\text { DEPREC } \\
\text { G \& A } \\
\text { OTHER }\end{array}$ & $\begin{array}{l}1.5 \\
.1 \\
.6 \\
.4\end{array}$ & $\begin{array}{r}1.5 \\
.1 \\
.5 \\
.7\end{array}$ & $\begin{array}{r}1.7 \\
.1 \\
.7 \\
.5\end{array}$ & $\begin{array}{r}1.9 \\
.1 \\
.8 \\
.5\end{array}$ & $\begin{array}{r}2.0 \\
.1 \\
.8 \\
.5\end{array}$ & $\begin{array}{r}2.2 \\
.1 \\
.9 \\
.6\end{array}$ & $\begin{array}{r}2.4 \\
.01 \\
1.0 \\
.6\end{array}$ & $\begin{array}{l}2.6 \\
1.1 \\
1.0 \\
.7\end{array}$ & $\begin{array}{r}2.8 \\
1.1 \\
.7\end{array}$ \\
\hline TOTAL FC & 2.6 & 2.3 & 3.0 & 3.2 & 3.5 & 3.7 & 4.0 & 4.4 & 4.7 \\
\hline STARTUP COSTS & 0.0 & 0.3 & 0.0 & 0.0 & 0.0 & 0.0 & 0.0 & 0.0 & 0.0 \\
\hline TOTAL OPER COSTS & 14.2 & 15.3 & 16.6 & 17.9 & 19.3 & 20.8 & 22.5 & 24.3 & 26.2 \\
\hline OFERATING INCOME & 8.1 & 8.3 & 9.5 & 10.3 & 11.1 & 12.0 & 12.9 & 14.0 & 15.1 \\
\hline INTEREST & 1.4 & 1.1 & .9 & .6 & .3 & .3 & .3 & .3 & .3 \\
\hline INC. BEF. LCF/TAX & 6.8 & 7.6 & 8.6 & 9.7 & 10.8 & 11.7 & 12.6 & 13.7 & 14.8 \\
\hline LCF USED & 0.0 & 0.0 & 0.0 & 0.0 & 0.0 & 0.0 & 0.0 & 0.0 & 0.0 \\
\hline TAXABLE INCOME & 6.8 & 7.6 & 8.6 & 9.7 & 10.8 & 11.7 & 12.6 & 13.7 & 14.8 \\
\hline $\begin{array}{l}\text { ITC USED } \\
\text { TAXES PAID }\end{array}$ & $\begin{array}{l}0.0 \\
3.4\end{array}$ & $\begin{array}{l}0.0 \\
3.8\end{array}$ & $\begin{array}{l}0.0 \\
4.3\end{array}$ & $\begin{array}{l}0.0 \\
4.8\end{array}$ & $\begin{array}{l}0.0 \\
5.4\end{array}$ & $\begin{array}{l}0.0 \\
5.9\end{array}$ & $\begin{array}{l}0.0 \\
6.3\end{array}$ & $\begin{array}{l}0.0 \\
6.8\end{array}$ & $\begin{array}{l}0.0 \\
7.4\end{array}$ \\
\hline INCOME AFTER TAX & 3.4 & 3.8 & 4.3 & 4.8 & 5.4 & 5.9 & 6.3 & 6.8 & 7.4 \\
\hline $\begin{array}{l}\text { DEPREC } \\
\text { CHG IN OTH ASSEIS } \\
\text { PRINC REFAY } \\
\text { CHG IN WC } \\
\text { CHG IN BAN LINE } \\
\text { ENG COST } \\
\text { CONSTR COST } \\
\text { CONSTR INT } \\
\text { DEETT FIN }\end{array}$ & $\begin{array}{r}0.1 \\
0.0 \\
-1.4 \\
-.2 \\
.1 \\
0.0 \\
0.0 \\
0.0 \\
0.0\end{array}$ & $\begin{array}{r}.1 \\
0.0 \\
-1.0 \\
-.2 \\
.1 \\
0.0 \\
0.0 \\
0.0 \\
0.0\end{array}$ & $\begin{array}{r}.1 \\
0.0 \\
-1.9 \\
-.2 \\
.1 \\
0.0 \\
0.0 \\
0.0 \\
0.0\end{array}$ & $\begin{array}{r}.1 \\
0.0 \\
-2.2 \\
-.2 \\
.1 \\
0.0 \\
0.0 \\
0.0 \\
0.0\end{array}$ & $\begin{array}{l}.1 \\
0.0 \\
0.0 \\
-.2 \\
.1 \\
0.0 \\
0.0 \\
0.0 \\
0.0\end{array}$ & $\begin{array}{l}.1 \\
0.0 \\
0.0 \\
-.2 \\
0.1 \\
0.0 \\
0.0 \\
0.0 \\
0.0\end{array}$ & $\begin{array}{l}.1 \\
0.0 \\
0.0 \\
-.2 \\
.1 \\
0.0 \\
0.0 \\
0.0 \\
0.0\end{array}$ & $\begin{array}{l}.1 \\
0.0 \\
0.0 \\
\cdots .3 \\
.1 \\
0.0 \\
0.0 \\
0.0 \\
0.0\end{array}$ & $\begin{array}{l}.1 \\
0.0 \\
0.0 \\
-.3 \\
.1 \\
0.0 \\
0.0 \\
0.0 \\
0.0\end{array}$ \\
\hline $\begin{array}{l}\text { ANINUAL CASH FLOW } \\
\text { CINMUL CASH FLOWW }\end{array}$ & $\begin{array}{r}2.0 \\
13.2\end{array}$ & $\begin{array}{r}2.2 \\
15.4\end{array}$ & $\begin{array}{r}2.4 \\
17.7\end{array}$ & $\begin{array}{r}2.6 \\
20.3\end{array}$ & $\begin{array}{r}5.4 \\
25.7\end{array}$ & $\begin{array}{r}5.8 \\
31.5\end{array}$ & $\begin{array}{r}6.3 \\
37.8\end{array}$ & $\begin{array}{r}6.8 \\
44.6\end{array}$ & $\begin{array}{r}7.3 \\
51.9\end{array}$ \\
\hline
\end{tabular}


FINANCIAL ANALYSIS OF SUN BELT ENERGY CORPORATION FROJECT

CORN - TO-ETHANOL PLANT

4.8 MIL GPY RATED CAFACITY

ASSUMFTIONS (1982\$)

14285 GPD RATEN CAP

$100 \times$ ACT CAF

333 DIAYS PER YEAR
PERCENT LT DEBT
LT DEET INT RATE
i6.0\% $\%$

$\begin{array}{ll}\text { LT DEBT INT RATE } & 16.0 \% \\ \text { LT DEBT REP.AY } & 10 \% \mathrm{YFS}\end{array}$

GANK LINE INT RT $16.0 \%$

IIATE OF ANALYSIS: 29/03/82

CAPITAL COSTS

CONSTR INT. RATE

$12.4 \mathrm{MIL}$
$16.0 \%$

ELECTR SALES PRICE
ELECTR PURCH COST

$5.0 \mathrm{C} / \mathrm{KWH}$

NET ETH PRICE $.90 \$ / G A L$

DDG PRICE $170.00 \$ / T O N$

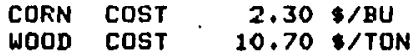

DAYS REC ETH/DFY AG 30

$\begin{array}{ll}\text { DAYS REC ETH/DEY AG } & 30 \\ \text { DAYS REC DDG } & 30 \\ \text { DAYS REC ELECTR } & 30\end{array}$

DAYS PAY CORN

DAYS PAY CORN
DAYS FAY WOOD

S.0 C/KWH

$\begin{array}{ll}\text { ETH REAL ESC } & 0.0 x \\ \text { CORN \& DDG REAL ESC } & 0.0 \%\end{array}$

ELECTR REAL ESC

$0.0 x$

$\begin{array}{ll}\text { INFLATION RATE } & 8.0 \% \\ \text { CAP COST REAL ESC } & 5.0 \%\end{array}$

FED INC TAX

STATE INC TAX

STATE SALES TAX EXE,TPT

STATE GASOLINE EXCI JE TAX EXEMPT

FED GASOLINE EXCISE TAX EXEMPT

$-\quad 0$
0
LIFO INUENTORY POLICY
15 DAYS ETH STORAGE
15 DAYS CORN STORAGE

15 DAYS WOOD STORAGE

\section{RESULTS}

ROE $12.8 \times$ NPV (25 $\times$ DISC KATE) -3.3 MIL FAYGACK 1993

1.0 IN OPER YR 1

1.5 IN OPER YR 6

ENO COSTS \$. .6 MIL

INITIAL DEET \$12.4 MIL

MAXIMUM CASH EQUITY $\$ 7.5 \mathrm{MIL}$

ANNUAL SALES:

4.8 MIL GaLS ETH

16649 TONS DNG

0.0 MW ELECT

ANNUAL FLANT KEQS

1.9 MIL BU CORN

6 MIL KWH

29273 ACRES CORN 166 MIL LE STEAM

16649 HEAD CẠTtLE 
INCOME AND CASH FLOW (\$ MIL)

\begin{tabular}{|c|c|c|c|c|c|c|c|c|c|}
\hline REUENUES & 1982 & 1983 & 1984 & 1985 & 1986 & 1987 & 1988 & 1989 & 1990 \\
\hline $\begin{array}{l}\text { ETHANOL } \\
\text { DDG } \\
\text { DRY AGENT } \\
\text { ELECT PWR }\end{array}$ & $\begin{array}{l}0.0 \\
0.0 \\
0.0 \\
0.0\end{array}$ & $\begin{array}{l}0.0 \\
0.0 \\
0.0 \\
0.0\end{array}$ & $\begin{array}{l}0.0 \\
0.0 \\
0.0 \\
0.0\end{array}$ & $\begin{array}{r}7.8 \\
3.6 \\
.3 \\
0.0\end{array}$ & $\begin{array}{r}0.4 \\
3.9 \\
.4 \\
0.0\end{array}$ & $\begin{array}{l}9.1 \\
4.2 \\
.4 \\
0.0\end{array}$ & $\begin{array}{l}9.8 \\
4.5 \\
.4 \\
0.0\end{array}$ & $\begin{array}{r}10.6 \\
4.9 \\
.4 \\
0.0\end{array}$ & $\begin{array}{r}11.4 \\
5.2 \\
.5 \\
0.0\end{array}$ \\
\hline TOTAL REUS & 0.0 & 0.0 & 0.0 & 11.7 & 12.6 & 13.6 & 14.7 & 15.9 & 17.2 \\
\hline $\begin{array}{l}\text { VARIABLE COST } \\
\text { FEEISTOCK } \\
\text { WOOD } \\
\text { DRY AGENT } \\
\text { ELECT } \\
\text { OTHER }\end{array}$ & $\begin{array}{l}0.0 \\
0.0 \\
0.0 \\
0.0 \\
0.0\end{array}$ & $\begin{array}{l}0.0 \\
0.0 \\
0.0 \\
0.0 \\
0.0\end{array}$ & $\begin{array}{l}0.0 \\
0.0 \\
0.0 \\
0.0 \\
0.0\end{array}$ & $\begin{array}{r}5.5 \\
.5 \\
.3 \\
.4 \\
.6\end{array}$ & $\begin{array}{r}6.0 \\
.5 \\
.4 \\
.4 \\
.7\end{array}$ & $\begin{array}{l}6.4 \\
.6 \\
.4 \\
.4 \\
.7\end{array}$ & $\begin{array}{l}6.9 \\
.6 \\
.4 \\
.5 \\
.8\end{array}$ & $\begin{array}{l}7.5 \\
.7 \\
.4 \\
.5 \\
.9\end{array}$ & $\begin{array}{r}8.1 \\
.7 \\
.5 \\
.5 \\
.9\end{array}$ \\
\hline TOTAL VC & 0.0 & 0.0 & 0.0 & 7.3 & 7.9 & 8.6 & 9.2 & 10.0 & 10.8 \\
\hline $\begin{array}{l}\text { FIXED COSTS } \\
\text { LABOR } \\
\text { DEPREC } \\
\text { G \& A A } \\
\text { OTHER }\end{array}$ & $\begin{array}{l}0.0 \\
0.0 \\
0.0 \\
0.0\end{array}$ & $\begin{array}{l}0.0 \\
0.0 \\
0.0 \\
0.0\end{array}$ & $\begin{array}{l}0.0 \\
0.0 \\
0.0 \\
0.0\end{array}$ & $\begin{array}{r}.9 \\
3.3 \\
.4\end{array}$ & $\begin{array}{r}1.0 \\
3.3 \\
.4 \\
.3\end{array}$ & $\begin{array}{r}1.1 \\
3.3 \\
.4 \\
.3\end{array}$ & $\begin{array}{r}1.2 \\
3.3 \\
.5 \\
.3\end{array}$ & $\begin{array}{r}1.3 \\
3.2 \\
.5 \\
.3\end{array}$ & $\begin{array}{r}1.4 \\
.1 \\
.6 \\
.4\end{array}$ \\
\hline TOTAL FC & 0.0 & 0.0 & 0.0 & 4.9 & 5.0 & 5.1 . & 5.2 & 5.4 & .2 .4 \\
\hline STARTUP COSTS & 0.0 & 0.0 & .7 & 0.0 & 0.0 & 0.0 & 0.0 & 0.0 & 0.0 \\
\hline TOTAL OPER COSTS & 0.0 & 0.0 & .7 & 12.2 & 12.9 & 13.7 & 14.5 & 15.4 & 13.2 \\
\hline OPERATING INCOME & 0.0 & 0.0 & -.7 & -.5 & -.3 & -.0 & .2 & .5 & 4.0 \\
\hline INTEREST & 0.0 & 0.0 & 0.0 & 2.1 & 2,0 & 1.9 & 1.8 & 1.7 & 1.5 \\
\hline INC, BEF, LCF/TAX & 0.0 & 0.0 & -.7 & -2.7 & -2.3 & -2.0 & -1.6 & -1.1 & 2.5 \\
\hline LCF USED & 0.0 & 0.0 & 0.0 & 0.0 & 0.0 & 0.0 & 0.0 & 0.0 & 2.5 \\
\hline TAXABLE INCOME & 0.0 & 0.0 & -.7 & -2.7 & -2.3 & -2.0 & -1.6 & -1.1 & 0.0 \\
\hline $\begin{array}{l}\text { ITC USED } \\
\text { TAXES PAID }\end{array}$ & $\begin{array}{l}0.0 \\
0.0\end{array}$ & $\begin{array}{l}0.0 \\
0.0\end{array}$ & $\begin{array}{l}0.0 \\
0.0\end{array}$ & $\begin{array}{l}0.0 \\
0.0\end{array}$ & $\begin{array}{l}0.0 \\
0.0\end{array}$ & $\begin{array}{l}0.0 \\
0.0\end{array}$ & $\begin{array}{l}0.0 \\
0.0\end{array}$ & $\begin{array}{l}0.0 \\
0.0\end{array}$ & $\begin{array}{l}0.0 \\
0.0\end{array}$ \\
\hline INCOME AFTER TAX & 0.0 & 0.0 & -.7 & -2.7 & -2.3 & -2.0 & -1.6 & -1.1 & 2.5 \\
\hline $\begin{array}{l}\text { DEPREC } \\
\text { CHG IN OTH ASSETS } \\
\text { PRINC REPAY } \\
\text { CHG IN WC } \\
\text { CHG IN BANK LINE } \\
\text { ENG COST } \\
\text { CONSTR COST } \\
\text { CONSTR INT } \\
\text { DEBT FIN }\end{array}$ & $\begin{array}{l}0.0 \\
0.0 \\
0.0 \\
0.0 \\
0.0 \\
-.6 \\
0.0 \\
0.0 \\
0.0\end{array}$ & $\begin{array}{r}0.0 \\
0.0 \\
0.0 \\
0.0 \\
0.0 \\
0.0 \\
-9.4 \\
-.7 \\
7.6\end{array}$ & $\begin{array}{r}0.0 \\
-1.2 \\
0.0 \\
-1.7 \\
.08 \\
0.0 \\
-4.6 \\
-1.9 \\
4.8\end{array}$ & $\begin{array}{l}3.3 \\
0.0 \\
-.6 \\
-.1 \\
.0 \\
0.0 \\
0.0 \\
0.0 \\
0.0\end{array}$ & $\begin{array}{l}3.3 \\
0.0 \\
-.7 \\
-.1 \\
0.0 \\
0.0 \\
0.0 \\
0.0 \\
0.0\end{array}$ & $\begin{array}{l}3.3 \\
0.0 \\
-.8 \\
-.1 \\
.0 \\
0.0 \\
0.0 \\
0.0 \\
0.0\end{array}$ & $\begin{array}{l}3.3 \\
0.0 \\
-.9 \\
-.1 \\
.1 \\
0.0 \\
0.0 \\
0.0 \\
0.0\end{array}$ & $\begin{array}{r}3.2 \\
0.0 \\
-1.1 \\
-.1 \\
. .1 \\
0.0 \\
0.0 \\
0.0 \\
0.0\end{array}$ & $\begin{array}{r}.1 \\
0.0 \\
-1.2 \\
-.1 \\
.1 \\
0.0 \\
0.0 \\
0.0 \\
0.0\end{array}$ \\
\hline $\begin{array}{l}\text { ANNUAL CASH FLOW } \\
\text { CUMUL CASIS FLOW }\end{array}$ & $\begin{array}{l}-.6 \\
-.6\end{array}$ & $\begin{array}{l}-2.5 \\
-3.1\end{array}$ & $\begin{array}{l}-4.3 \\
-7.5\end{array}$ & $\begin{array}{r}.0 \\
-7.4\end{array}$ & $\begin{array}{r}.2 \\
-7.2\end{array}$ & $\begin{array}{r}.5 \\
-6.7\end{array}$ & -6.7 & $\begin{array}{r}1.0 \\
-5.0\end{array}$ & $\begin{array}{r}1.3 \\
-3.7\end{array}$ \\
\hline
\end{tabular}


INCOME AND CASH FLOW (\$ MIL)

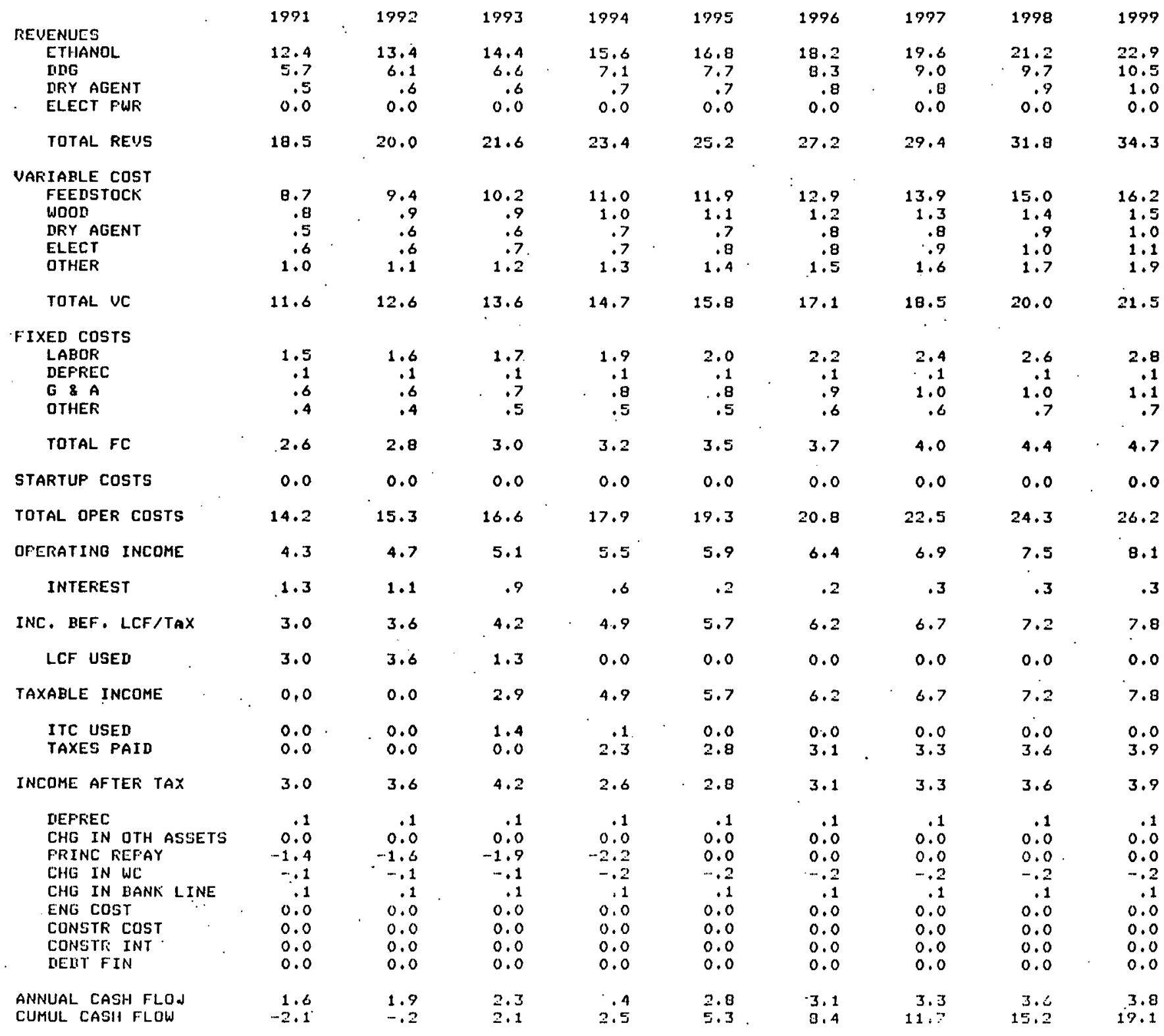


CORN - TU-ETHANOL FLANT

4.8 NISL GFY KATE:D CAFACITY

ASSUMPTIONS (1782

14285 GPD RATED CAF
333 DAYS PER YEAR
FUEL: WOON

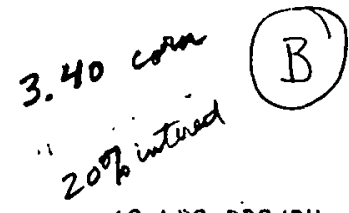

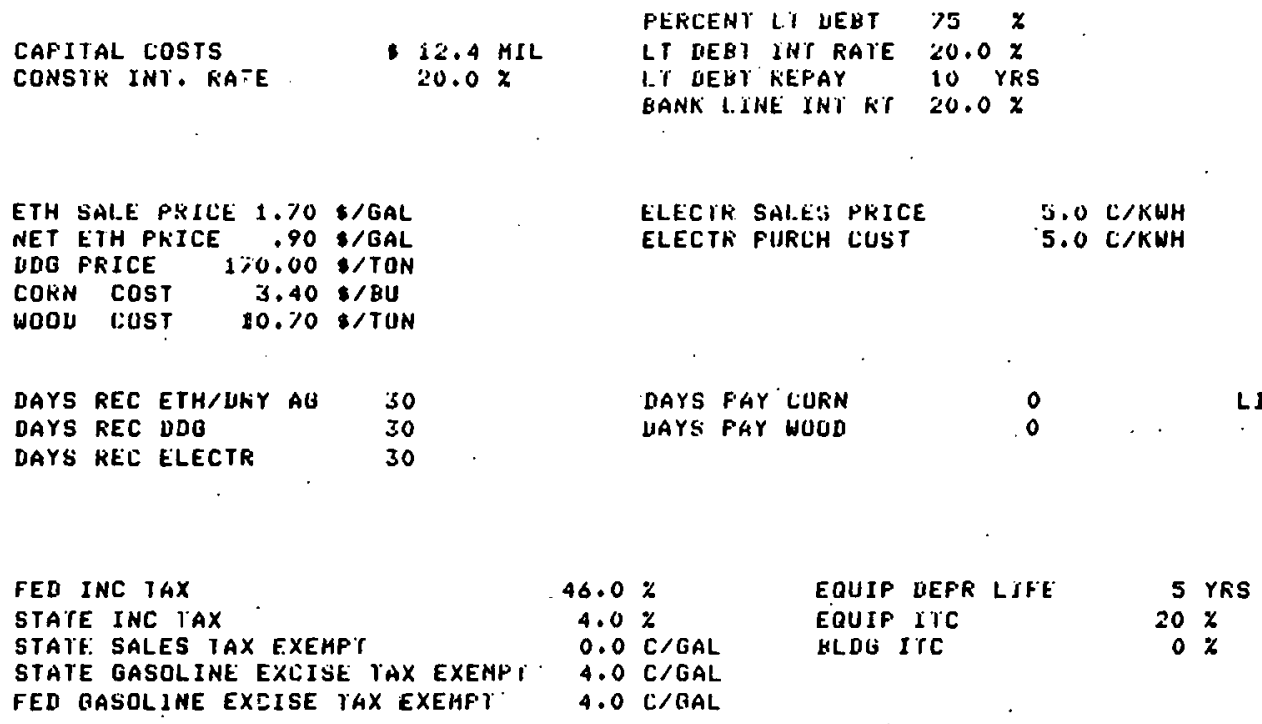

$\begin{array}{ll}\text { INFLATION RATE } & 8.0 \% \\ \text { LAP COS'T REAL ESC } & 5.0 \%\end{array}$

ETH KEAL ESC

CORN \& DDG REAL ESC $0.0 x$

$\begin{array}{ll}\text { ELECTK KEAL ESC } & 0.0 \% \\ \text { WOOU KEAL ESC } & 0.0 \%\end{array}$

LIFO INUENTORY POLICY

15 DAYS ETH SIORABE

15 DAYS CORN STORAG

15 MAYS DUG STORAGE

RESULTS

ROE $0.0 \div$ NFU ( $Z E *$ DISL RATE) -7.9 KXL . FAYBACK

DEBI COV: $\quad .8$ IN OPER YK 1 .5 IN OFEK YK 6

ENG CUSTS .6 MIL INITIAL UEBT $12.9 \mathrm{MIL}$

MAXIMUI LASH EQUITY $7.8 \mathrm{MIL}$

ANNUAL SALES:

4.8 HIL GALS ETH

16649 RINS DDE

0.0 HW ELECT

ANNUAL FLANT KEOS

1.9 HIL BU LURN

6 MIL KWH

2927.3 ACRES CORN

1.S6 TIL LQ STEAN

16649 HEALI CATTLE 
INCOME AND LASH FL.Oti (\& HJL)

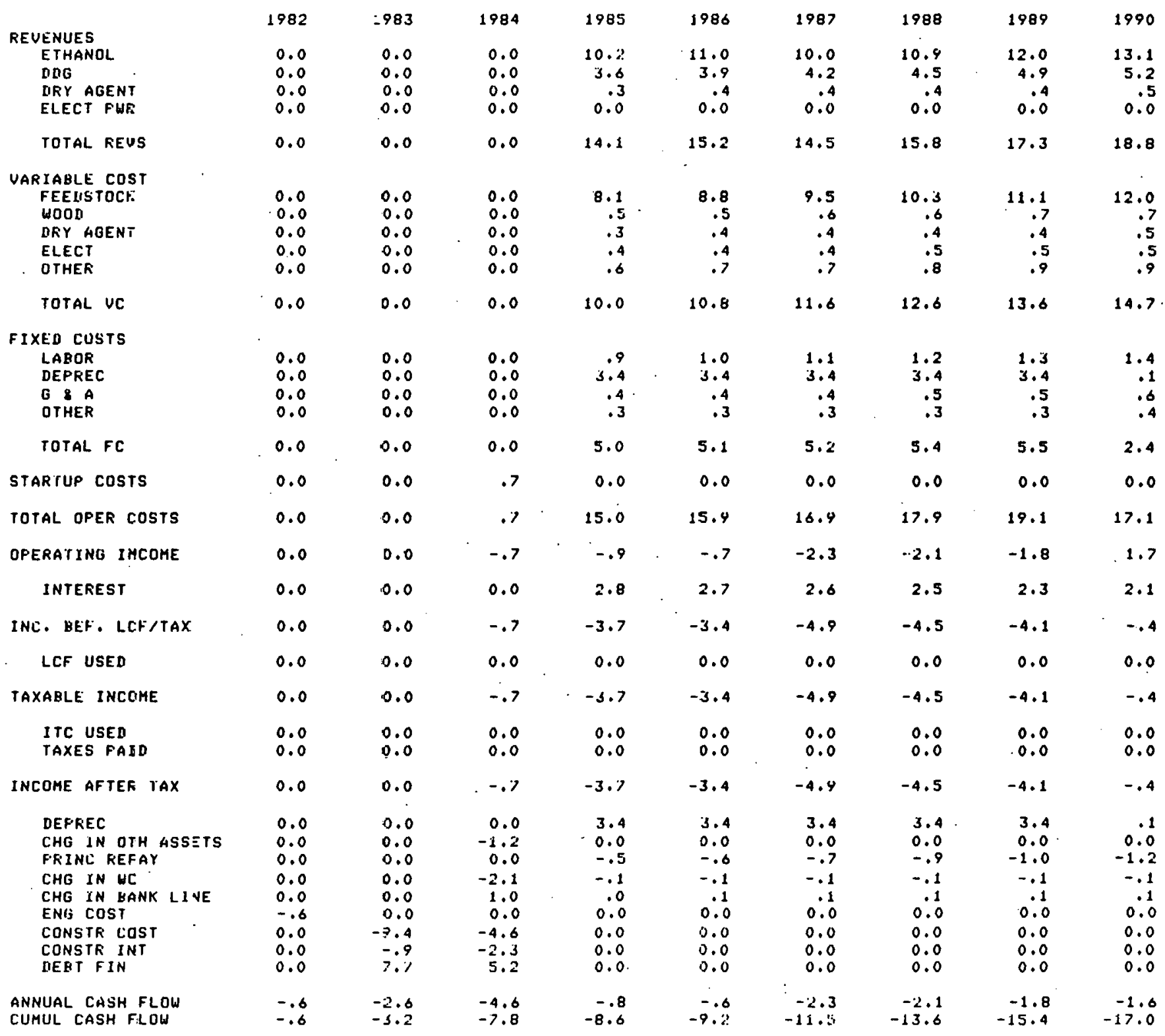


INCOME ANH CASH FIOOW (\$ KIL)

\begin{tabular}{|c|c|c|c|c|c|c|c|c|c|}
\hline REVENUES & 1991 & 1992 & 1993 & 1994 & $1998 \mathrm{j}$ & 1996 & 1997 & 1998 & 1999 \\
\hline ETHANOL & 14.3 & $15 . \AA$ & 17.0 & 18.8 & 20.1 & 21.4 & 23.7 & 25.8 & 28.0 \\
\hline DDG & 5.7 & 6.2 & 6.6 & 7.1 & 7.7 & 8.3 & 9.0 & 9.7 & 10.5 \\
\hline $\begin{array}{l}\text { DRY AQENT } \\
\text { ELECT PUR }\end{array}$ & 0.5 & 0.0 & 0.0 & 0.7 & 0.0 & 0.8 & $\begin{array}{l}.8 \\
0.0\end{array}$ & $\begin{array}{r}.9 \\
0.0\end{array}$ & $\begin{array}{l}1.0 \\
0.0\end{array}$ \\
\hline TOTAL REUS & 20.4 & 22.2 & 24.2 & 26.2 & 28.5 & 30.4 & 33.6 & 36.4 & 39.5 \\
\hline UARIABLE COST. & & & & & & & & & \\
\hline FEEDSTOCK & 12.9 & 14.0 & 15.1 & 16.3 & 17.6 & 19.0 & 20.5 & 22.2 & 23.9 \\
\hline DRY AOENT & .5 &.$\epsilon$ & .6 & 1.0 & 107 & 2.8 & 1.3 & 1.4 & 1.5 \\
\hline ELECT & .6 &.$e$ & .7 & .7 & .8 & .8 & $\begin{array}{l}.8 \\
.9\end{array}$ & $\therefore 8$ & 1.0 \\
\hline OTHER & 1.0 & 1.1 & 1.2 & 1.3 & 1.4 & 1.5 & 1.6 & 1.7 & 1.9 \\
\hline TOTAL UC & 15.8 & 17.1 & 18.5 & 19.9 & 21.5 & 23.3 & 25.1 & 27.1 & 29.3 \\
\hline FIXED COSTS & & & & & & & & & \\
\hline LAROR & 1.5 & 1.6 & 1.7 & 1.9 & 2.0 & 2.2 & 2.4 & 2.6 & 2.8 \\
\hline DEPREC & .1 & .1 & .1 & .1 & .1 & .1 & .1 & .1 & .1 \\
\hline 684 & .6 & .6 & .7 & .8 & .8 & .9 & 1.0 & 1.0 & 1.1 \\
\hline OTHER & .4 & .4 & .5 & .5 & .5 & .6 & .6 & .7 & .7 \\
\hline TOTAL FC & 2.6 & 2.8 & 3.0 & 3.2 & 3.5 & 3.7 & 4.0 & 4.4 & 4.7 \\
\hline STARTUP COSTS & 0.0 & 0.0 & 0.0 & 0.0 & 0.0 & 0.0 & 0.0 & 0.0 & 0.0 \\
\hline TOIAL UPER COSTS & 18.4 & 19.9 & 21.4 & 23.2 & 20.0 & 27.0 & 29.2 & 31.5 & 34.0 \\
\hline OPERATING INCOHE & 2.0 & 2.4 & 2.7 & 3.1 & 3.5 & 3.9 & 4.4 & 4.9 & 5.5 \\
\hline INTEREST & 1.9 & 1.6 & 1.2 & .8 & .3 & .4 & .4 & .4 & .4 \\
\hline INC. BEF. LCF/TAXX & .2 & .8 & 1.5 & 2.3 & 3.1 & 3.6 & 4.0 & 4.5 & 5.0 \\
\hline LCF USED & .2 & .8 & 1.5 & 2.3 & 3.1 & 3.6 & 4.0 & 4.5 & 1.8 \\
\hline TAXABLE INCOME & 0.0 & 0.0 & 0.0 & 0.0 & 0.0 & 0.0 & 0.0 & 0.0 & 3.2 \\
\hline $\begin{array}{l}\text { ITC USED } \\
\text { IAXES FAID }\end{array}$ & $\begin{array}{l}0.0 \\
0.0\end{array}$ & $\begin{array}{l}0.0 \\
0.0\end{array}$ & $\begin{array}{l}0.0 \\
0.0\end{array}$ & $\begin{array}{l}0.0 \\
0.0\end{array}$ & $\begin{array}{l}0.0 \\
0.0\end{array}$ & $\begin{array}{l}0.0 \\
0.0\end{array}$ & $\begin{array}{l}0.0 \\
0.0\end{array}$ & $\begin{array}{l}0.0 \\
0.0\end{array}$ & $\begin{array}{l}1.6 \\
0.0\end{array}$ \\
\hline INCOME AFTER TAX & .2 & .8 & 1.5 & 2.3 & 3.1 & 3.6 & 4.0 & 4.5 & 5.0 \\
\hline DEPREC & .1 & .1 & .1 & .1 & .1 & .1 & .1 & .1 & .1 \\
\hline CHG IN OTH ASSETS & 0.0 & 0.0 & 0.0 & 0.0 & 0.0 & 0.0 & 0.0 & 0.0 & 0.0 \\
\hline PRINC REFAY & -1.5 & -1.8 & -2.1 & -2.6 & 0.0 & 0.0 & 0.0 & 0.0 & 0.0 \\
\hline CHB IN WC & -.2 & -.2 & -.2 & -.2 & -.2 & -.2 & -.2 & -.3 & -.3 \\
\hline $\begin{array}{l}\text { CHG IN HANK LINE } \\
\text { ENG COST }\end{array}$ & .1 & .1 & $\therefore 1$ & .1 & .1 & $\cdot 1$ & .1 & .1 & .1 \\
\hline $\begin{array}{l}\text { ENE COST } \\
\text { CONSTR COST }\end{array}$ & $\begin{array}{l}0.0 \\
0.0\end{array}$ & $\begin{array}{l}0.0 \\
0.0\end{array}$ & 0.0 & $\begin{array}{l}0.0 \\
0.0\end{array}$ & $\begin{array}{l}0.0 \\
0.0\end{array}$ & 0.0 & 0.0 & 0.0 & 0.0 \\
\hline CONSTR INT & 0.0 & 0.0 & 0.0 & 0.0 & 0.0 & 0.0 & 0.0 & 0.0 & 0.0 \\
\hline DEET FIN & 0.0 & 0.0 & 0.0 & 0.0 & 0.0 & 0.0 & 0.0 & 0.0 & 0.0 \\
\hline ANNUAL I:ASH FLOW & $-1 \cdot 3$ & -1.0 & -.7 & -.3 & 3.1 & 3.5 & 4.0 & 4.5 & 5.0 \\
\hline CUHUL CASH FLUW & -18.4 & -19.3 & -20.0 & 20.3 & -17.2 & $-23,1$ & $-9, \%$ & -5.3 & -.3 \\
\hline
\end{tabular}


FINANCIAL ANALYSIS OF SUN BELT ENERGY CORFORATION FROJECT

CORN - TO-ETHANOL PLANT

4.8 MIL GPY RATED CAFACITY

ASSUMPTIONS ( $190: \$)$

14285 GFI RATEI CAP

$100 \%$ ACT CAP

333 DAYS FER YEAR

$\begin{array}{lll}\text { PERCENT LT DEBT } & 75 & \chi \\ \text { LT DEBT INT RATE } & 20.0 \% \\ \text { LT IEBT REPAY } & 8 \\ \text { BANK LINE INT RT } & 20.0 \%\end{array}$

CONSTR INT. RATE

\section{$12.4 \mathrm{MIL}$}

BANK LINE INT RT

$20.0 \%$

ETH SALE PRICE 1,70 \$/GAL

NET ETH FRICE .90 \$/GAL

DDG FRICE $170.00 \$ T$ TON

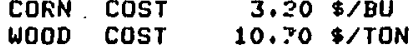

ELECTR SALES FRICE

ELECTR FURCH COST

$5.0 \mathrm{C} / \mathrm{KWH}$

$5.0 \mathrm{C} / \mathrm{KWH}$

DAYS PAY CORN

DAYS PAY WOOU

0

DAYS REC DDG

30

30

FED INC TAX

STATE INC TAX

STATE SALES TAX EXEMPT

FED GASOLINE EXCISE TAX EXEMPT

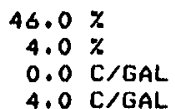

$0.0 \mathrm{C} / \mathrm{GAL}$

4,0 C/GAL
4.0 C/GAL

\section{RESULTS}

DEBT COU:

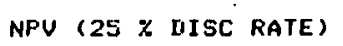

PAYBACK

6 IN DPER YK 6

ENG COSTS $.6 \mathrm{NIL}$

INITIAL DEBT

. $12.4 \mathrm{MIL}$

ANNUAL SALES:

4.8 MIL GALS ETH

16649 TONS DNG

EQUIP DEPR LIFE

EQUIP ITC

DATE OF ANALYSIS:

FUEL: WOOD

$31 / 03 / 82$

$.5 . \mathrm{GAL}$ ETH/BU

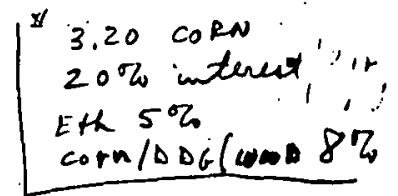

18 LAS ING/BU
ANNUAL PLANT RERS

AII FULL CAPACITY $1.9 \mathrm{MIL}$ EU CORN
$6 \mathrm{MIL} \mathrm{KWH}$
29273 ACRES CORN 166 MIL LE STEAM
INFLATION RATE

CAP COST REAL ESC

$5.0 x$

$5.0 \%$ $\begin{array}{ll}\text { ETH REAL ESC } & 0.0 x \\ \text { CORN } 8 \text { DDG REAL ESC } & 3.0 x\end{array}$

$\begin{array}{ll}\text { CORN \& DDG REAL ESC } & 3.0 \% \\ \text { ELECTR REAL ESC } & 0.0 \% \\ \text { WOOD REAL ESC } & 3.0 \%\end{array}$

$3.0 x$

LIFO INUENTORY POLICY

15 DAYS ETH STORAGE

15 DAYS CORN STORAGE

15 DAYS WOOD STORAGE

. 


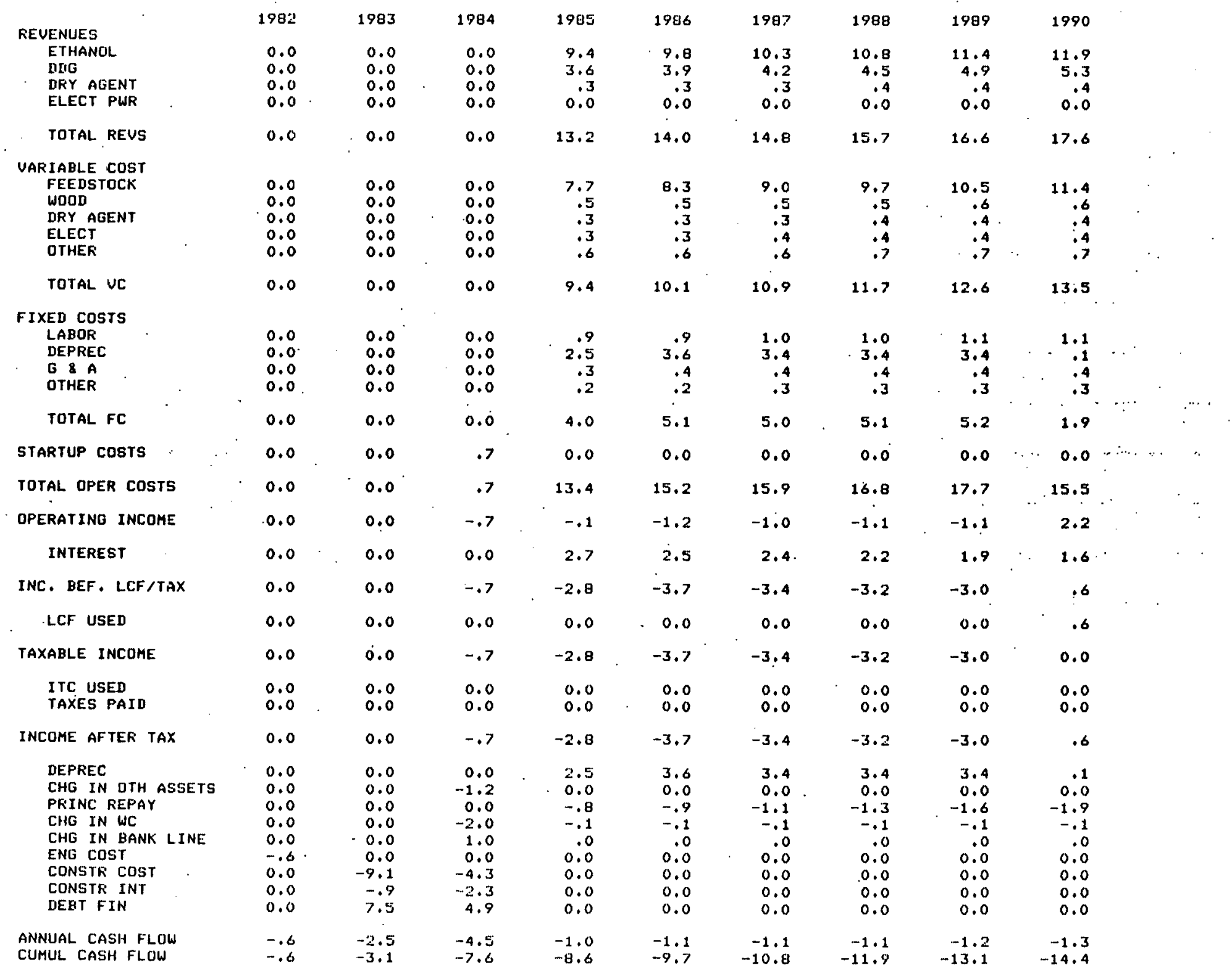


INCOME ANI CASH FLOW (\$ MIL)

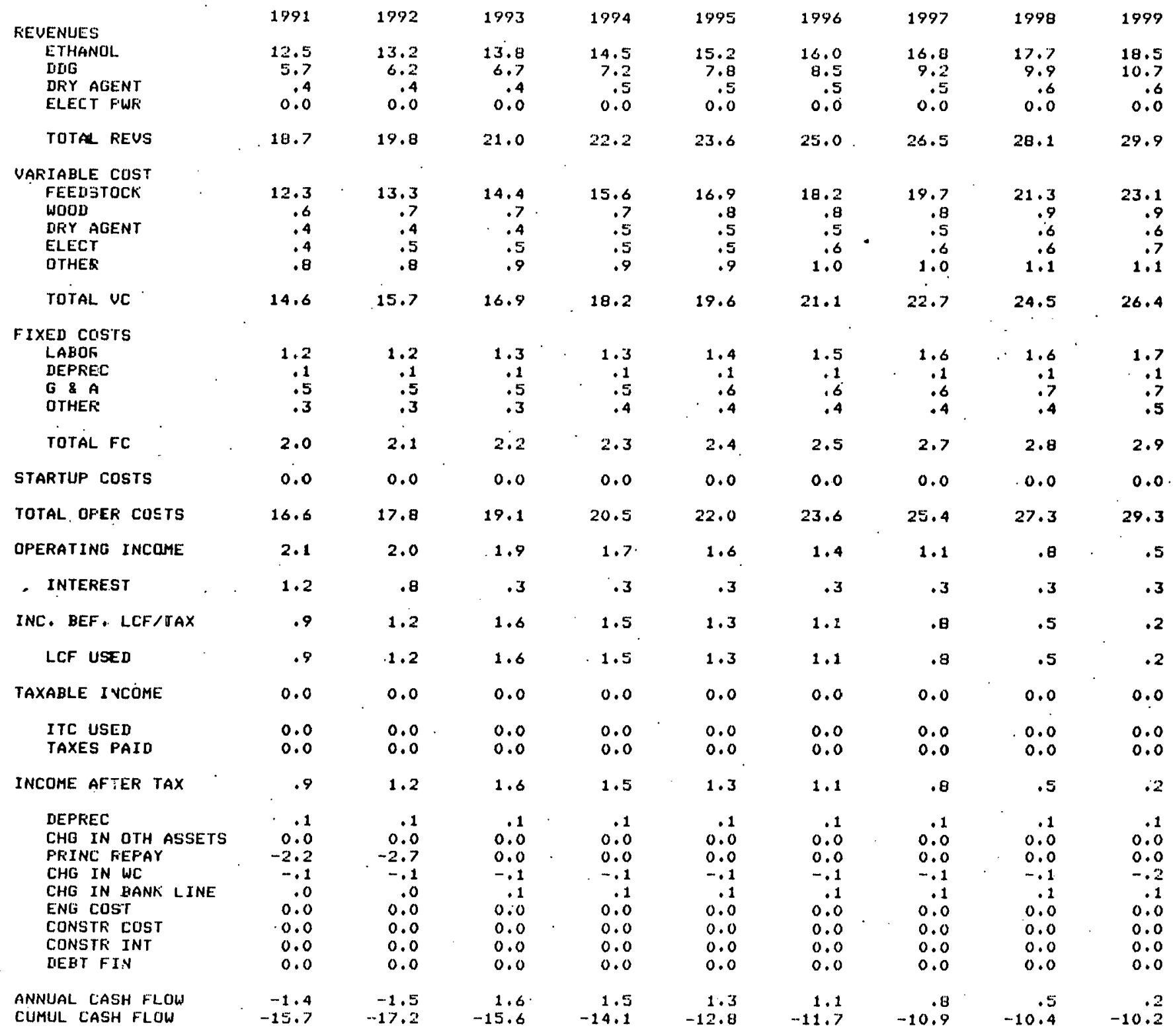

